\title{
Mineralogy and Geochemistry of Some Belt Rocks, Montana and Idaho
}

\author{
By J. E. HARRISON and D. J. GRIMES
}

CONTRIBUTIONS TO ECONOMIC GEOLOGY

GE OLOGICAL S U R VEY B ULLETIN 1312 - O

A comparison of rocks from two widely separated areas in Belt terrane

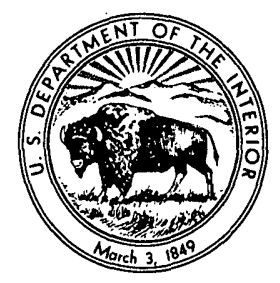




\section{UNITED STATES DEPARTMENT OF THE INTERIOR \\ WALTER J. HICKEL, Secretary}

\section{GEOLOGICAL SURVEY}

William T. Pecora, Director

Library of Congress catalog-card No. 75-607766 


\section{CONTENTS}

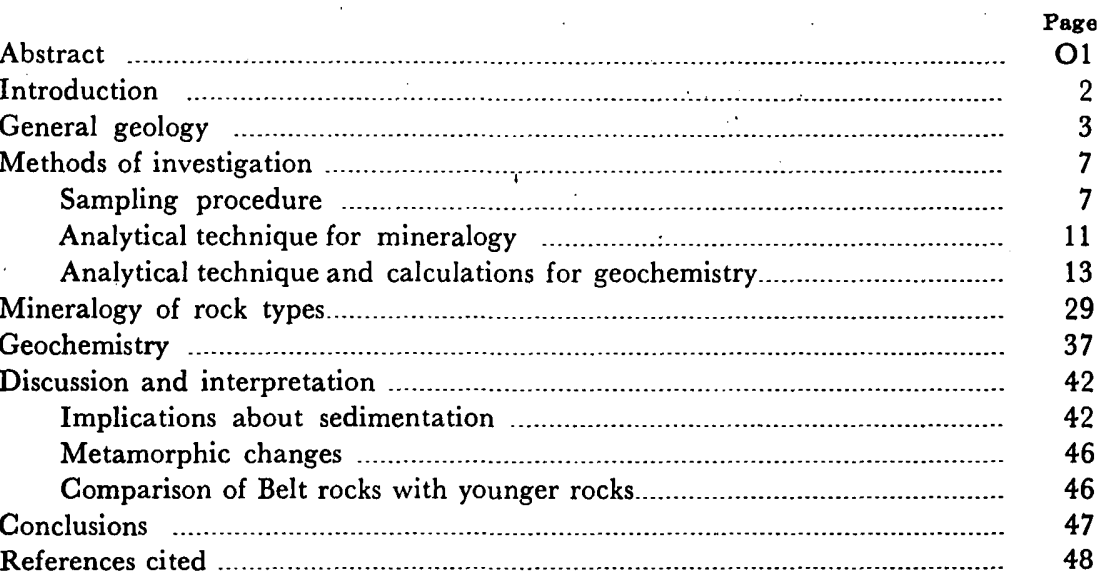

\section{ILLUSTRATIONS}

Figure 1. Geologic map of northern Idaho and northwestern Montana

2. Correlation chart for part of the Belt Supergroup, northern Idaho and western Montana.

3. Geologic sketch map of the Pend Oreille area, showing sample localities and numbers

4. Histograms showing precision of semiquantitative spectrographic analyses of some Belt rocks

5. Diagram showing average modes of rocks of the Belt Supergroup

6. Diagram showing ratio among quartz, potassium feldspar, and plagioclase in siltites

7. Diagram showing average mineralogy of clastic Belt rocks............ 37

8. Diagram showing geometric means of semiquantitative spectrographic analyses of different Belt rock types 
TABLE 1. Sample distribution by area, formation, rock type, and metamorphic grade

$\mathrm{O} 10$

2. Average modes of Belt rocks from the Mission Mountains Primitive Area

3. Semiquantitative spectrographic analyses of Belt rocks from the Pend Oreille area

4. Replicate semiquantitative spectrographic analyses of Belt rocks from the Pend Oreille area

5. Replicate analyses of standard sample G-1

6. Mean element content of low-grade Belt rocks from the Mission Mountains Primitive Area

7. Mean element content of Belt rocks from the Pend Oreille area

8. Numbers used to replace nonnumeric codes $(\mathrm{N}, \mathrm{L}$, and $>)$ prior to geochemical calculations

9. Relative concentration of elements in the heavy mineral fraction of some Belt rocks from the Pend Oreille area

10. Average granite and average composition of the Ganadian Shield compared with average Belt rock from the Pend Oreille area.

11. Average minor element content of pelitic rocks of different ages and geologic environments. 


\title{
CONTRIBUTIONS TO ECONOMIC GEOLOGY
}

\section{MINERALOGY AND GEOCHEMISTRY OF SOME BELT ROGKS, MONTANA AND IDAHO}

\author{
By J. E. HARrison and D. J. Grimes
}

\begin{abstract}
The mineralogy and geochemistry of about 20,000 feet of Belt rocks in the Mission Mountains Primitive Area, northwestern Montana, are compared with those of about 40,000 feet of Belt rocks in the Pend Oreille area, northern Idaho and northwestern Montana. Semiquantitative spectrographic analyses of 254 samples from the Mission Mountains and 230 samples from the Pend Oreille area form the basis for chemical comparison; quantitative X-ray mineralogic analyses of most samples provide the mineralogic data. Both analytical techniques are rapid and inexpensive.

All rocks were classified on the basis of grain size, texture, and carbonate content into one of four rock types: argillitic rocks (shale or mudstone equivalents), siltites (siltstone equivalents), quartzites (sandstone equivalents), and carbonate rocks. The semiquantitative spectrographic method has good precision for all rock types, but the accuracy is only fair for rocks of moderate to high carbonate content.

Comparison of clastic rocks from both areas shows the mineralogy to be very similar even to the ratio among quartz, potassium feldspar, and plagioclase in siltites. Most rocks are graywacke or like graywacke. Mineralogy is, not surprisingly, directly related to grain size; for example, we find that from argillitic rocks to siltites to quartzites the amount of quartz increases, the amount of feldspar remains about the same, and the illite-sericite-chlorite content decreases.

Several elements show distinct patterns of abundance related to the rock types. Elements directly related in abundance to amount of illite-sericite-chlorite in clastic rocks are $\mathrm{B}, \mathrm{Co}, \mathrm{Cr}, \mathrm{Ni}, \mathrm{Sc}, \mathrm{V}, \mathrm{Fe}, \mathrm{Mg}, \mathrm{Ti}$, and possibly $\mathrm{La}$ and $\mathrm{Y}$. Siltites and quartzites contain $\mathrm{Cu}$ in sulfide grains and flakes, and $\mathrm{Cu}$ is distinctly more abundant in those rocks or in fossil algal forms (usually calcareous or dolomitic siltite) in the Mission Mountains. Carbonate rocks or rocks containing moderately abundant carbonate minerals show increased amounts of $\mathrm{Ca}, \mathrm{Mg}, \mathrm{Mn}, \mathrm{Sr}$, and $\mathrm{Pb}$. The higher $\mathrm{B}$ content of certain Pend Oreille area strata and the higher $\mathrm{Co}, \mathrm{Cu}$, and $\mathrm{Ni}$ contents of Mission Mountains strata seem most likely to reflect differences in source area of the original sediments. If so, the $\mathrm{Cu}$ abundance and distribution identified in this study may aid in the search for stratabound copper deposits in Belt rocks.

The excellent sorting of grain sizes in similar strata through tens of thousands of feet of rock in two widely separated areas, the remarkable uniformity of simple miner-
\end{abstract}


alogy, the apparently simple chemistry of depositional environments, and the bulk chemistry of the rocks suggest that the weathering and transportation processes of Belt time resulted in homogenization of clastic components drawn from large granitic source areas. This homogenization requires a chemical weathering of most mafic minerals, a low terrane and low stream gradient, a nearly uniform availability of many minor elements for absorption on or inclusion in clay minerals, and a slow isostatic adjustment between source areas and basins of deposition during the several hundred million years of Belt rock deposition. Sedimentation processes in Belt time were not complicated by living plants or animals on the land or by significant life in the sea. Organic compounds did not complicate chemical environments of deposition.

Regional metamorphism from the chlorite-sericite zone of the greenschist facies into the biotite zone in the Pend Oreille area appears to have resulted only in a loss of $\mathrm{B}$ among the elements studied. Contact metamorphism, also in the biotite zone, resulted in an increase in $\mathrm{Ca}$ and $\mathrm{Sr}$, a decrease in $\mathrm{Ni}$, and a possible decrease in Co and B.

\section{INTRODUCTION}

Much attention has been given to Belt rocks during the past decade. Some of the interest has come from general recognition that the vast area of exposed Belt rocks represents one of the most poorly understood depositional basins in the United States; other interest is based on tectonic history yet to be deciphered; and more recent impetus for expanded study of Belt rocks comes from active exploration for stratabound copper deposits. So much new stratigraphic, structural, and chemical information has been gained recently that a compendium of data representing the state of knowledge only a few years ago (Ross, 1963) is rapidly becoming outdated.

This report discusses the mineralogy and minor element content of Belt rocks from two widely separated areas in the Idaho-Montana part of Belt terrane shown in figure 1. The data, accumulated from 1957 through 1968, represent samples collected for two purposes: a study of metamorphic effects in the Pend Oreille area and an evaluation of mineral potential in the Mission Mountains Primitive Area. The advent of reasonably precise and rapid semiquantitative spectrographic analyses, plus the availability of electronic computers to handle efficiently large masses of data, has permitted the geochemical study described here.

Several colleagues aided in collecting data included in this report. D. A. Jobin collected some samples from the Pend Oreille area and made some heavy mineral separations. M. W. Reynolds collected about half the samples from the Mission Mountains Primitive Area. Uteana Oda, now deceased, analyzed spectrographically the heavy and light mineral fractions. Paul W. Schmidt and George M. Fairer made X-ray spectrographic analyses of samples for the quantitative mineralogic studies. We are particularly grateful for the aid and counsel of Albert P. Marranzino on the spectrographic parts of the study. The manuscript benefited from reviews and discussions with M. R. Mudge and Jon Connor. 


\section{GENERAL GEOLOGY}

Rocks of the Belt Supergroup crop out over much of western Montana and northern Idaho (fig. 1) and northward into Canada. Equivalent Belt rocks extend southward from Missoula, Mont., for at least 150 miles, and scattered outcrops are known in northeastern Washington near the IdahoWashington State line. The limits of the basin, or basins, of deposition of Belt rocks are poorly known, although evidence indicates that the thrust zone on the eastern edge of Belt terrane (fig. 1) may mark the depositional edge of Belt rocks (Mudge, 1970). The western edge of the Belt terrane is so complicated by extensive intrusion and faulting that it has not yet been satisfactorily determined whether some high-grade metamorphic rocks in this area are Belt or pre-Belt. Between the thrust belt on the east and a Cretaceous batholith in the Idaho Panhandle (fig. 1), Belt rocks either are nearly unmetamorphosed or are not above the greenschist facies. The grade of regional metamorphism progressively increases from east to west and with depth in the stratigraphic section, as demonstrated by illite transformations to the 2M polymorph (Maxwell and Hower, 1967).

Belt rocks are generally monotonous in appearance because of fine grain size and drab color. The bulk of the supergroup has a grain size of silt or clay, and medium sand is the coarsest grain size observed in thousands of feet of rock over large areas. Quartzites and relatively pure to impure dolomites and minor amounts of limestones are scattered through the supergroup, although the carbonate rocks are uncommon in the lower part. Some carbonate rocks contain fossil algal forms, which are the only megascopic evidence of Precambrian life in the rocks. All rocks are dense and hard, reflecting the widespread low-grade metamorphism of most of the supergroup. Because of the metamorphism in most rocks of the Pend Oreille area and in some rocks of the Mission Mountains area, we use terms such as argillite, siltite, and quartzite rather than claystone, shale, siltstone, and sandstone to describe the rocks. Mudstone equivalents in the Belt Supergroup include argillite, silty argillite, and laminated argillite and siltite. Descriptions of rock types and formations for the Pend Oreille area are given by Harrison and Jobin (1963) and for the Mission Mountains Primitive Area by Harrison, Reynolds, Kleinkopf, and Pattee (1969). Most rocks contain shallow-water structures, although the abundance of such structures decreases noticeably in the lower parts of the section. Disconformities in the supergroup are difficult to identify, and angular unconformities are rare.

The stratigraphy of both areas is reasonably well known, and map units are traceable for many miles from each area. Correlation between the areas is still somewhat unreliable for a few members or formations (see queries in fig. 2), although for most it is adequate (fig. 2).

Obradovich and Peterman (1968) stated that the Belt rocks are from about $900 \mathrm{~m}$. y. (million years) to more than 1,300 m.y. old. The bulk of the 


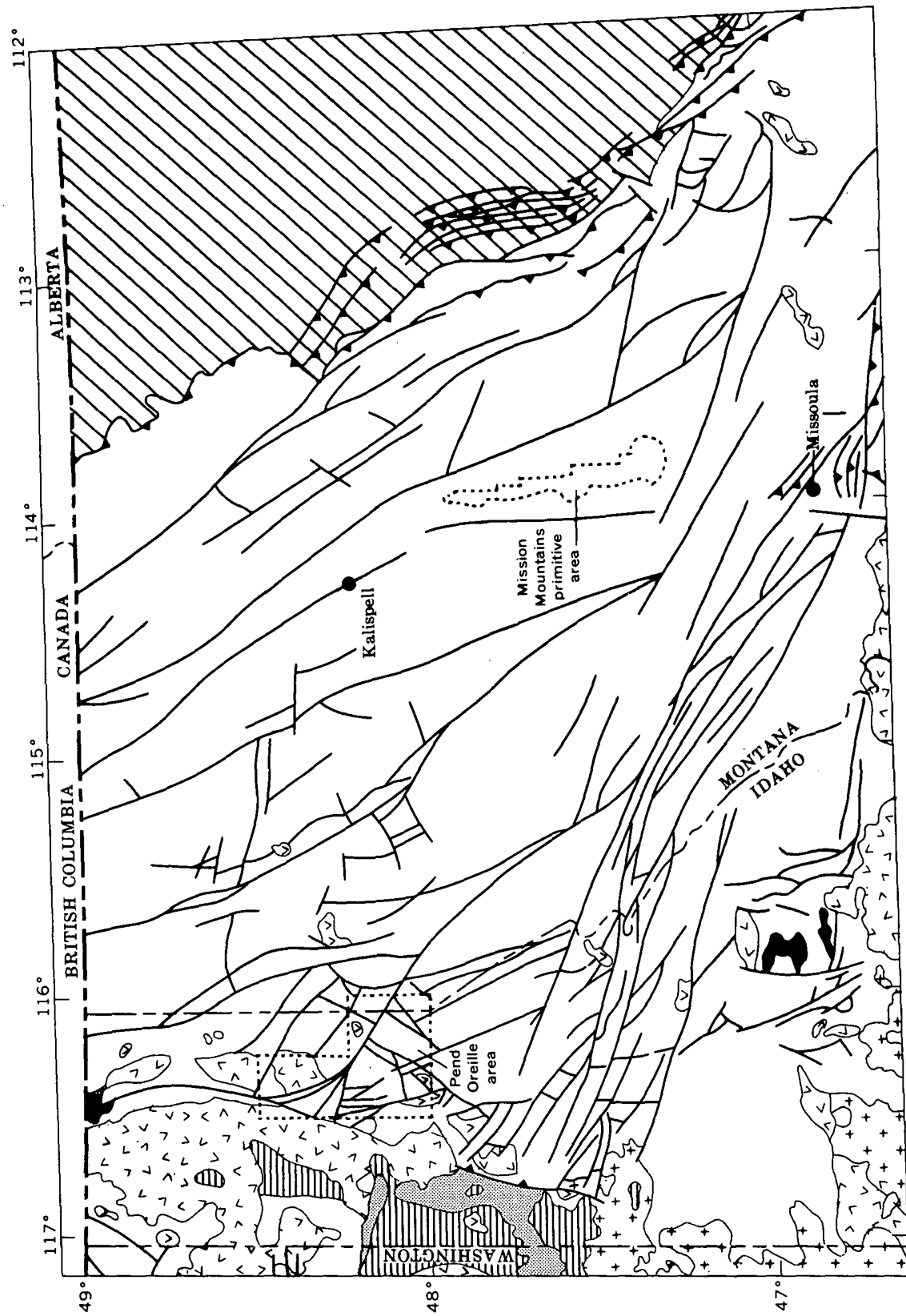




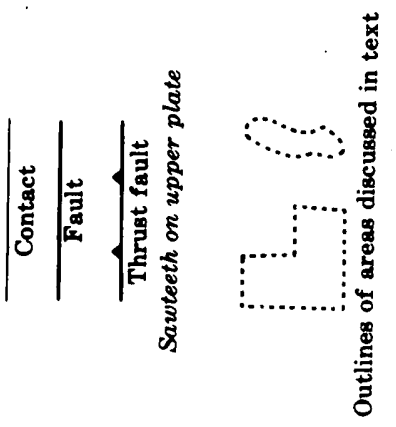

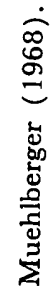

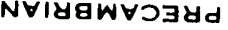

สู

交

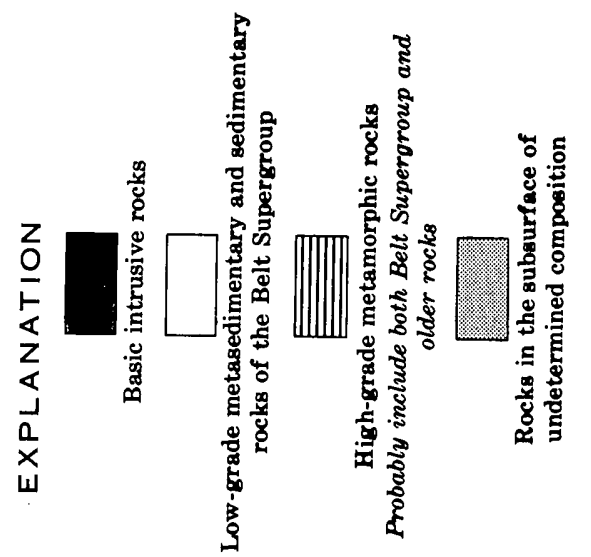

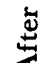

帘

:

ᄉㅂ

งกดヨว

롤

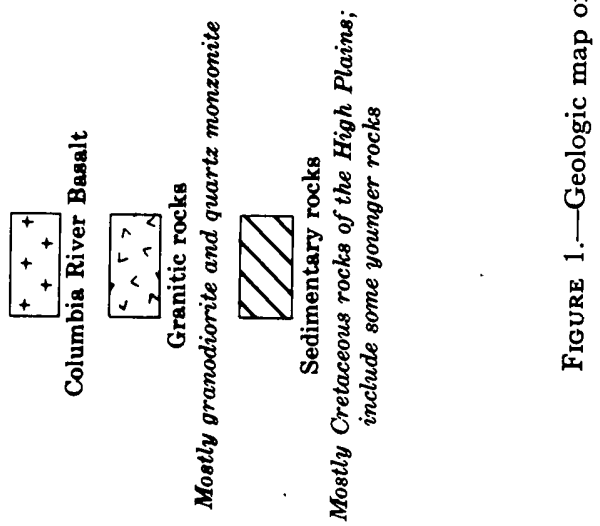




\begin{tabular}{|c|c|c|}
\hline $\begin{array}{c}\text { PEND OREILLE } \\
\text { AREA } \\
\text { (Harrison and Camp- } \\
\text { bell, 1963) }\end{array}$ & $\begin{array}{c}\text { MISSION MOUN- } \\
\text { TAINS PRIMITIVE } \\
\text { AREA } \\
\text { (Harrison and } \\
\text { others, 1969) } \\
\end{array}$ & $\begin{array}{c}\text { CAMP CREEK, } \\
\text { SOUTHERN LEWIS } \\
\text { AND CLARK RANGE } \\
\text { (MCGILL and } \\
\text { Sommers, 1967) } \\
\end{array}$ \\
\hline $\begin{array}{l}\text { Top eroded } \\
\text { Libby } \mathrm{Fm} \\
1,900+\mathrm{ft}\end{array}$ & & $\begin{array}{c}\text { Garnet Range Fm } \\
985+\mathrm{ft}\end{array}$ \\
\hline $\begin{array}{c}\text { (McNamara Fm } \\
\text { equivalent missing) }\end{array}$ & & $\begin{array}{c}\text { McNamara Fm } \\
3,070 \mathrm{ft}\end{array}$ \\
\hline $\begin{array}{c}\text { Member } 4 \\
700 \mathrm{ft}\end{array}$ & & $\begin{array}{c}\text { Bonner Qtz } \\
1,265 \mathrm{ft}\end{array}$ \\
\hline $\begin{array}{l}\frac{5}{2} \\
0 \\
4 \\
x \\
0 \\
0 \\
0\end{array}$ & $\begin{array}{l}\text { Shields Fm } \\
1,400+\mathrm{ft}\end{array}$ & $\begin{array}{c}\text { Shields Fm } \\
2,730 \mathrm{ft}\end{array}$ \\
\hline $\begin{array}{c}\text { Members } 3 \\
\text { and } 2 \\
700 \mathrm{ft}\end{array}$ & $\begin{array}{c}\text { Shepard Fm } \\
2,500 \mathrm{ft}\end{array}$ & $\begin{array}{c}\text { Shepard Fm } \\
1,980 \mathrm{ft}\end{array}$ \\
\hline $\begin{array}{l}\text { Member } 1 \\
600 \mathrm{ft}\end{array}$ & $\begin{array}{c}\text { Snowslip Fm } \\
3,800 \mathrm{ft}\end{array}$ & $\begin{array}{c}\text { Snowslip Fm } \\
2,235 \mathrm{ft}\end{array}$ \\
\hline $\begin{array}{c}\text { Wallace Fm } \\
10,200 \mathrm{ft}\end{array}$ & $\begin{array}{c}\text { Helena Fm } \\
9,800 \mathrm{ft}\end{array}$ & Base not exposed \\
\hline $\begin{array}{l}\text { St. Regis Fm } \\
1,100 \mathrm{ft}\end{array}$ & \multirow{5}{*}{$\begin{array}{l}\begin{array}{c}\text { Empire and Spokane } \\
\text { Fms } 2,300+\mathrm{ft}\end{array} \\
\text { Base not exposed }\end{array}$} & \\
\hline $\begin{array}{c}\text { Revett Fm } \\
2,000 \mathrm{ft}\end{array}$ & & \\
\hline $\begin{array}{c}\text { Burke Fm } \\
3,200 \mathrm{ft}\end{array}$ & & \\
\hline \multirow[t]{2}{*}{$\begin{array}{l}\text { Prichard Fm } \\
22,000+\mathrm{ft}\end{array}$} & & \\
\hline & & \\
\hline
\end{tabular}

Figure 2.-Correlation chart for part of the Belt Supergroup, northern Idaho and western Montana. 
Belt rocks exposed in the Mission Mountains Primitive Area (Helena Formation and strata above) result from a period of sedimentation about 1,100 m.y. old. Presumably, the lithostratigraphic equivalents of those rocks in the Pend Oreille area are approximately the same age. Rocks approximately 2,000 feet below the Helena and Wallace Formations are dated at about 1,300 m.y. at the eastern edge of the Belt terrane. Correlation of these dated rocks with those of the Pend Oreille area is not well established, but the general 1,800-m.y. age for the metamorphosed basement rocks in western Montana provides a maximum possible age of the Belt Supergroup.

Geologic differences and similarities between the two study areas are outlined in table 1, which lists samples collected according to area, formation, rock type, and metamorphic grade. The Mission Mountains Primitive Area contains strata probably laid down closer to shore than those of the Pend Oreille area, and subsequent diagenesis and metamorphism of the Mission Mountains strata were low grade. Many carbonate rocks in the Mission Mountains area are relatively pure, and carbonate cement is common. The Pend Oreille area contains strata whose relations to shore are less well defined. The stratigraphic units above the Wallace Formation are more similar in appearance to their equivalents in the Mission Mountains Primitive Area than are the Wallace and lower formations. Regional metamorphism is evident in the rocks in the Pend Oreille area; those rocks below the Revett Formation are clearly in the biotite zone of the greenschist facies. Effects of contact metamorphism, also of the biotite zone, are apparent near the granodiorite intrusions (fig. 3). Carbonate rocks are rarely pure, and carbonate cement or minerals are uncommon in most units.

\section{METHODS OF INVESTIGATION}

\section{SAMPLING PROCEDURE}

The sample localities for the Pend Oreille area are shown in figure 3, whereas those for the Mission Mountains are in a report by Harrison, Reynolds, Kleinkopf, and Pattee (1969, pl. 1B). The locality number on the maps is the same as the sample number listed in the tables of analytical data. The number of samples in table 1 is crudely proportional to the thickness and abundance of the rock types in a given formation. For the Mission Mountain samples, an exception to this generality is an overemphasis on quartzite and siltite in the Empire and Spokane and on quartzite in the Helena; stratabound copper minerals have been found in those rock types. Exceptions for the Pend Oreille samples are (1) a smaller than proportional number of samples of minor rock types in some formations, such as argillite in the Revett Formation and in member 4 of the Striped Peak Formation and quartzite in the Burke Formation, and (2) a larger than proportional number of samples for the Striped Peak because of the different character of each of its members. 


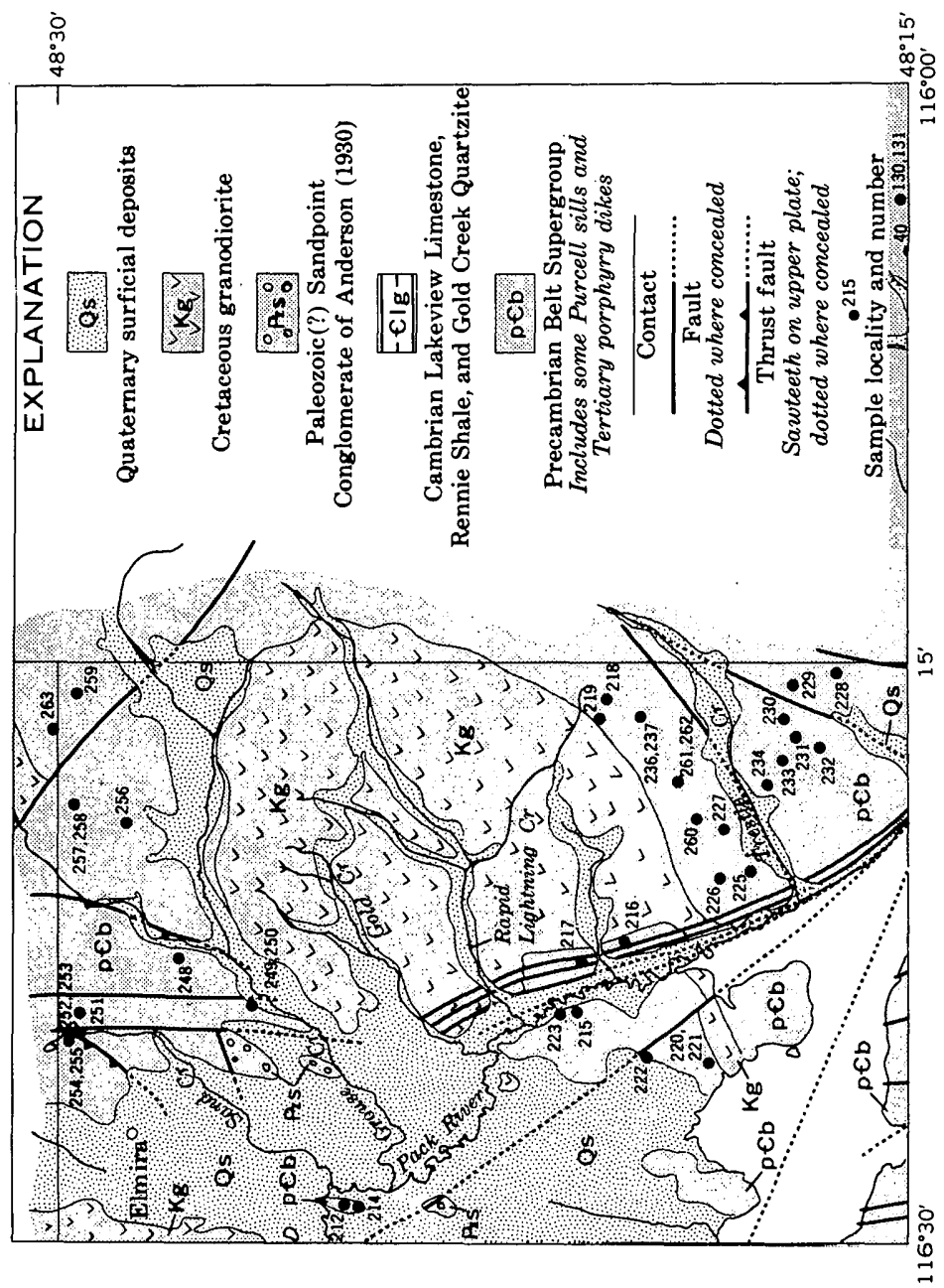




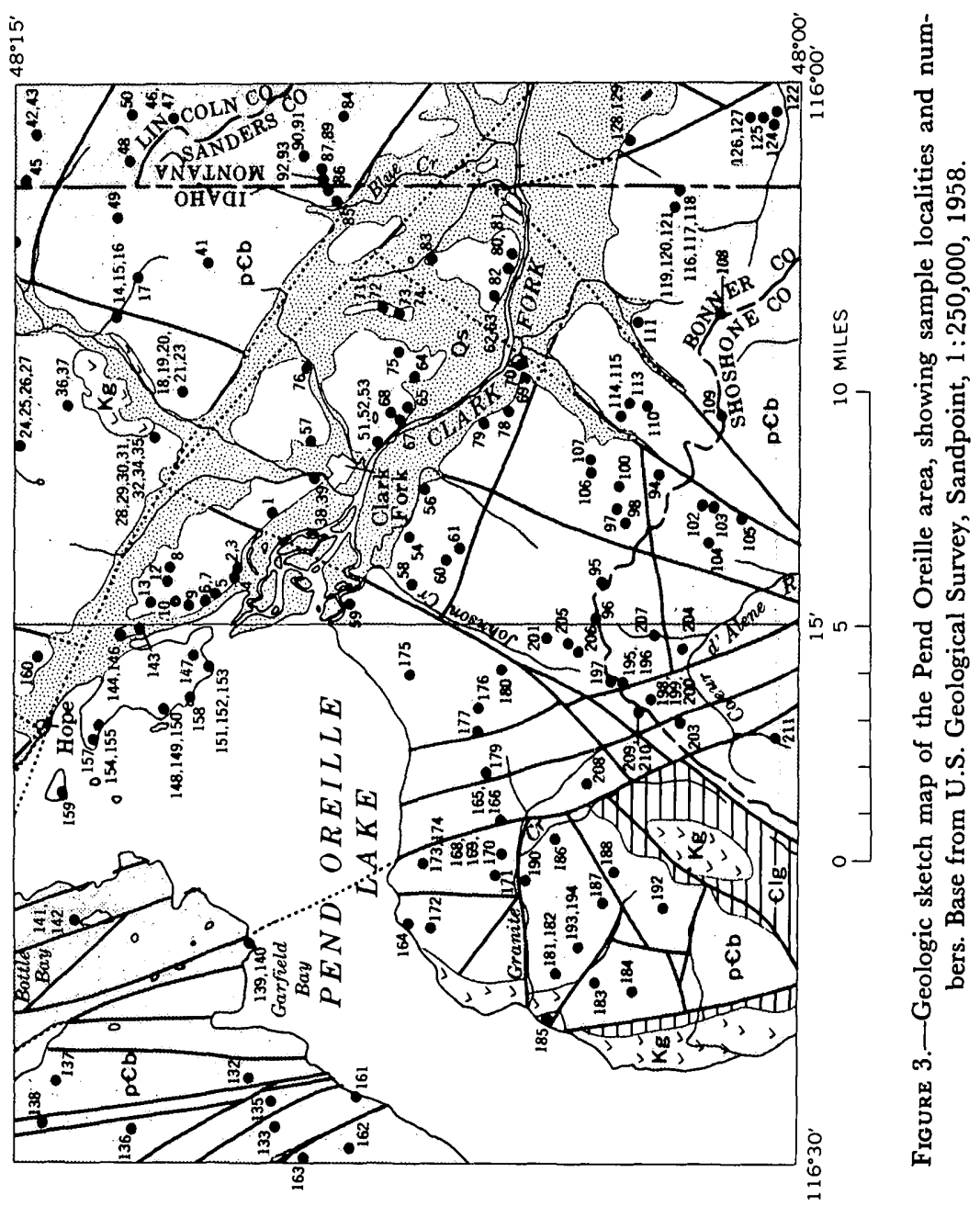




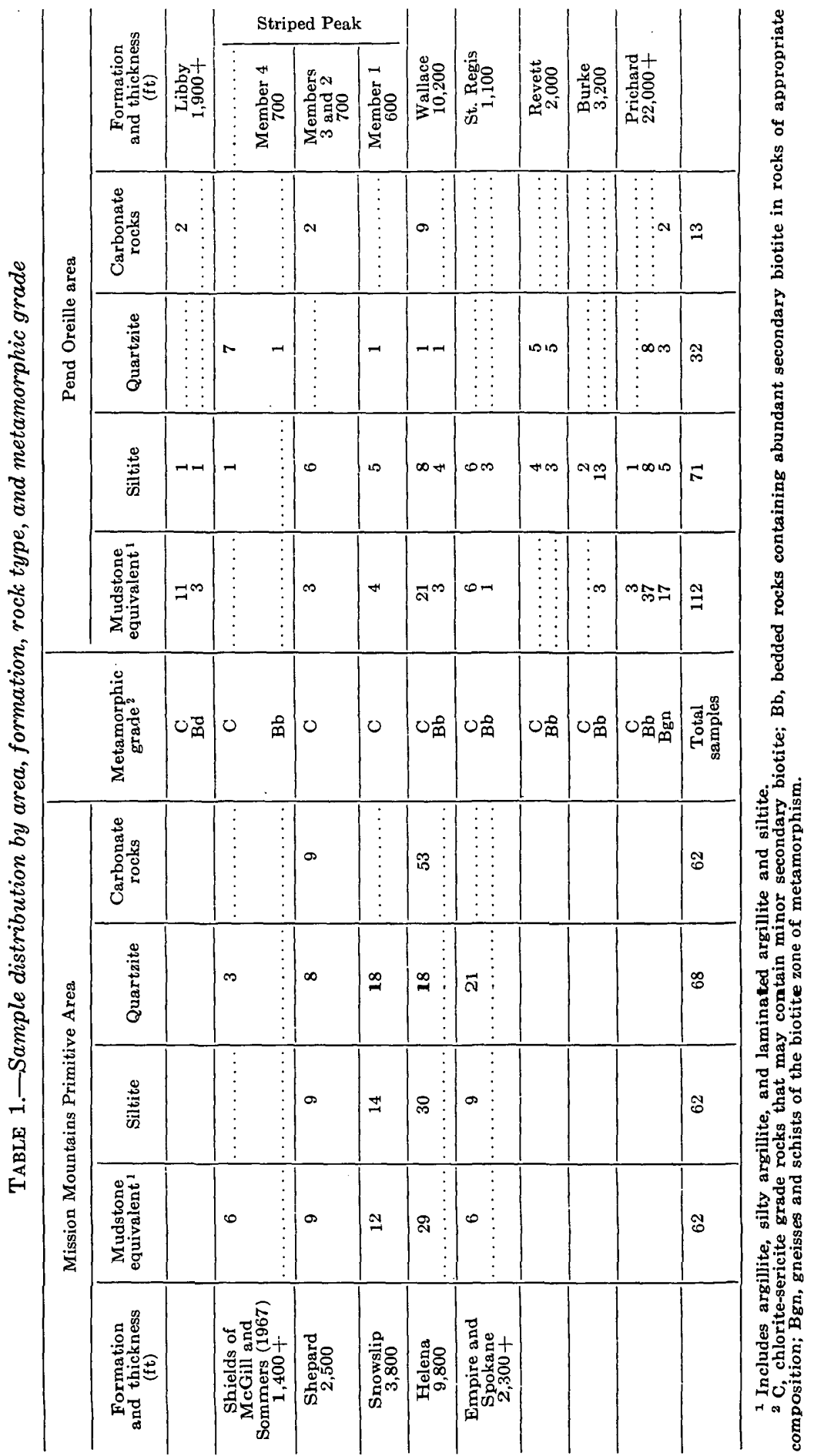


The field classification of the rocks into five rock types (argillite, laminated argillite and siltite, siltite, quartzite, and carbonate rocks) and into three metamorphic grades (simply defined as chlorite-sericite, biotite, and gneissic or schistose) was supported by study of thin sections of each sample from the Pend Oreille area and about half the samples from the Mission Mountains. The final classification for this study grouped most of the laminated rocks with the argillites into a rock group that is equivalent to mudstone.

\section{ANALYTICAL TECHNIQUE FOR MINERALOGY}

Mineralogical study of Belt rocks is complicated by (1) fine grain size, which makes microscopic modal analysis extremely difficult, and (2) low-grade metamorphic recrystallization, which effectively prevents simple separation into pure mineralogical components. Various staining techniques were tried in thin section, but they met with only partial success on the coarsest rocks; several standard mineral separation techniques were tried, again with only partial success in unmetamorphosed rocks. We resorted to $\mathrm{X}$-ray diffraction as a method for mineralogical analysis that would give comparable results in all rocks regardless of grain size or degree of metamorphism.

Quantitative mineralogic data for most Belt rocks can be obtained by $\mathrm{X}$-ray analysis. The simple mineral suite in most Belt rocks led Jobin and Harrison to experiment with quantitative X-ray spectrographic analyses in 1958. The first results of continuing studies were published a few years later (Harrison and Campbell, 1963). Studies by others-for example, Tatlock (1966), who made a thorough investigation of X-ray analysis of felsic rocks-verify that quantitative mineralogic data of even more complex rocks also can be obtained by X-ray analysis.

The analytical technique used for mineralogical determinations was specifically designed for Belt rocks and their low-grade metamorphic mineralogy. Replicate X-ray diffraction analyses on various rock types commonly fell within 5 percent of each other and always within 10 percent. Such precision is more than adequate for use in our studies because of the much greater differences observed among samples. In addition, the mineralogy was checked against partial or total chemical analyses for many samples with the result that the X-ray mineralogy appears to be not only reasonably precise but also reasonably accurate. All such chemical checks were within 10 percent of the X-ray values.

Each sample was crushed, ground for 15 minutes on a power mortar and pestle, spooned or dumped (to help avoid mica orientation) into an aluminum slotted powder holder, and run on the X-ray spectrometer from $2^{\circ}$ to $62^{\circ}$ at $2^{\circ} 2 \theta$ per minute. Peak heights above background on the spectrometer trace were then measured: the $10 \mathrm{~A}$ peak for mica (and illite), the 7 A peak for chlorite, the $3.35 \mathrm{~A}$ peak for quartz, the $3.25 \mathrm{~A}$ peak for 
potassium feldspar, the $3.2 \mathrm{~A}$ peak for plagioclase, the $3.05 \mathrm{~A}$ peak for calcite, and the $2.9 \mathrm{~A}$ peak for dolomite. The ratio of the $10 \mathrm{~A}$ mica peak and the $5 \mathrm{~A}$ mica peak was also determined.

General formulas for relating peak heights to mineralogy were devised by comparing X-ray spectrometer data with some complete chemical analyses, with $\mathrm{K}$ and $\mathrm{Na}$ analyses on most rocks, and with $\mathrm{Ca}$ and $\mathrm{Mg}$ analyses on carbonate rocks. In addition, estimates of biotite content were made from thin sections of all rocks showing visible metamorphic effects. Standard chemical formulas for each mineral were used to compute chemical content from rock mineralogy; the dark-green chlorite characteristic of the rocks was assumed to be iron rich, and the plagioclase (albite-oligoclase) was assumed to contain essentially all the $\mathrm{Na}$ in the rocks. From these data and assumptions, the following basic formulas for translating peak heights into quantitative mineralogy of nonbiotitic rocks emerged:

Quartz $=\frac{3.35 \mathrm{~A}-\mathrm{T} / 2(10 \mathrm{~A})}{4}$

(Subtraction required because of interference between main quartz peak and secondary mica peak);

Potassium feldspar $=\frac{3.25 \mathrm{~A}}{3}$;

Sericite (plus muscovite plus illite) $=\frac{2(10 \mathrm{~A})}{3}$;

Chlorite $=\frac{7 \mathrm{~A}}{2}$

Calcite $=\frac{3.05 \mathrm{~A}}{2}$;

Dolomite $=\frac{2.9 \mathrm{~A}}{3}$.

Estimates derived from these formulas were then recalculated to 100 percent. We consider that the simplified formulas given above are justified in this study because of the relatively simple mineralogy of the bulk of the Belt rocks and the limited intereference between principal and secondary peaks of the minerals. The ratio of the $10 \mathrm{~A}$ peak to the $5 \mathrm{~A}$ peak may be used to detect either preferred orientation of mica or presence of biotite in the sample. Biotite tends to give a higher $10 \mathrm{~A}$ and lower $5 \mathrm{~A}$ peak than an equal amount of sericite, whereas mica orientation gives an anomalously high 10 A peak. A microscopic scan of powdered rock or thin section usually distinguished which factor was affecting the normal peak ratio. Mica orientation necessitated a rerun of the sample; the detection of minor biotite required a decrease in the amount of the $10 \mathrm{~A}$ peak used in the calculation. Excessive biotite totally disrupts the mica formula, but the general formulas were found satisfactory for proportions of minerals other than mica. Mica content was determined from thin sections where biotite is a major constituent, where the metamorphic grade is relatively high, and where the grain size of the micas is sufficiently large. 
TABLE 2.-Average modes, in volume percent, of Belt rocks from the Mission Mountains Primitive Area

\begin{tabular}{|c|c|c|c|c|c|c|c|c|}
\hline Rock type 1 & $\begin{array}{c}\text { Number } \\
\text { of } \\
\text { samples }\end{array}$ & Quartz & $\begin{array}{l}\text { Plagio- } \\
\text { clase }\end{array}$ & $\begin{array}{l}\text { Potassium } \\
\text { feldspar }\end{array}$ & $\begin{array}{l}\text { Illite- } \\
\text { sericite }\end{array}$ & Chlorite & Calcite & Dolomite \\
\hline \multicolumn{9}{|c|}{ Shields Formation } \\
\hline $\begin{array}{c}\text { Dolomitic laminated } \\
\text { argillite and siltite. } \\
\text { Dolomitic siltite..... } \\
\text { Quartzite.......... }\end{array}$ & $\begin{array}{l}3 \\
2 \\
3\end{array}$ & $\begin{array}{l}41 \\
45 \\
73\end{array}$ & $\begin{array}{c}11 \\
15 \\
4\end{array}$ & $\begin{array}{l}6 \\
8 \\
2\end{array}$ & $\begin{array}{l}20 \\
12 \\
10\end{array}$ & $\begin{array}{l}9 \\
7 \\
9\end{array}$ & $\begin{array}{l}0 \\
0 \\
0\end{array}$ & $\begin{array}{r}13 \\
13 \\
2\end{array}$ \\
\hline \multicolumn{9}{|c|}{ Shepard Formation } \\
\hline $\begin{array}{l}\text { Carbonatic argillite or } \\
\text { laminated argillite } \\
\text { and siltite. } \\
\text { Carbonatic siltite.... } \\
\text { Carbonatic quartzite. } \\
\text { Impure limestone.... }\end{array}$ & $\begin{array}{l}5 \\
3 \\
2 \\
6 \\
\end{array}$ & $\begin{array}{l}38 \\
38 \\
44 \\
18 \\
\end{array}$ & $\begin{array}{l}16 \\
16 \\
22 \\
10\end{array}$ & $\begin{array}{c}3 \\
5 \\
4 \\
\text { Trace } \\
\end{array}$ & $\begin{array}{r}16 \\
13 \\
7 \\
9\end{array}$ & $\begin{array}{r}14 \\
9 \\
5 \\
8\end{array}$ & $\begin{array}{r}8 \\
7 \\
9 \\
53 \\
\end{array}$ & $\begin{array}{r}5 \\
12 \\
9 \\
2\end{array}$ \\
\hline \multicolumn{9}{|c|}{ Snowslip Formation } \\
\hline $\begin{array}{l}\text { Argillite or laminated } \\
\text { argillite and siltite. } \\
\text { Siltite............. } \\
\text { Quartzite........ }\end{array}$ & $\begin{array}{l}6 \\
5 \\
5\end{array}$ & $\begin{array}{l}38 \\
42 \\
58\end{array}$ & $\begin{array}{l}21 \\
22 \\
19\end{array}$ & $\begin{array}{l}1 \\
3 \\
0\end{array}$ & $\begin{array}{r}26 \\
24 \\
9\end{array}$ & $\begin{array}{r}11 \\
6 \\
6\end{array}$ & $\begin{array}{l}1 \\
3 \\
8\end{array}$ & $\begin{array}{c}1 \\
0 \\
\text { Trace }\end{array}$ \\
\hline \multicolumn{9}{|c|}{ Helena Formation } \\
\hline $\begin{array}{l}\text { Silty argillite......... } \\
\text { Calcareous silty }\end{array}$ & 5 & 33 & 14 & 3 & 29 & 13 & 7 & 1 \\
\hline $\begin{array}{l}\text { argillite. . . . . . } \\
\text { Carbonatic siltite.... } \\
\text { Calcareous quartzite. } \\
\text { Dolomitic limestone. }\end{array}$ & $\begin{array}{r}2 \\
13 \\
6 \\
17\end{array}$ & $\begin{array}{l}34 \\
33 \\
46 \\
17\end{array}$ & $\begin{array}{r}13 \\
14 \\
19 \\
6\end{array}$ & $\begin{array}{c}0 \\
3 \\
4 \\
\text { Trace }\end{array}$ & $\begin{array}{r}16 \\
17 \\
7 \\
8\end{array}$ & $\begin{array}{l}6 \\
6 \\
6 \\
4\end{array}$ & $\begin{array}{l}30 \\
15 \\
16 \\
51\end{array}$ & $\begin{array}{r}1 \\
12 \\
2 \\
14\end{array}$ \\
\hline \multicolumn{9}{|c|}{ Empire and Spokane Formations } \\
\hline $\begin{array}{l}\text { Carbonatic argillite or } \\
\text { argillite and siltite. } \\
\text { Calcareous siltite.... } \\
\text { Quartzite.......... }\end{array}$ & $\begin{array}{l}3 \\
2 \\
7\end{array}$ & $\begin{array}{l}27 \\
35 \\
62\end{array}$ & $\begin{array}{l}14 \\
18 \\
15\end{array}$ & $\begin{array}{l}0 \\
0 \\
0\end{array}$ & $\begin{array}{l}30 \\
20 \\
12\end{array}$ & $\begin{array}{r}15 \\
11 \\
6\end{array}$ & $\begin{array}{r}3 \\
12 \\
3\end{array}$ & $\begin{array}{r}11 \\
4 \\
2\end{array}$ \\
\hline \multicolumn{9}{|c|}{ All formations named above } \\
\hline $\begin{array}{l}\text { Carbonatic argillite or } \\
\text { argillite and siltite. } \\
\text { Carbonatic siltite.... } \\
\text { Carbonatic quartzite. } \\
\text { Carbonate rocks..... }\end{array}$ & $\begin{array}{l}24 \\
25 \\
23 \\
23\end{array}$ & $\begin{array}{l}35 \\
36 \\
57 \\
18\end{array}$ & $\begin{array}{r}16 \\
16 \\
16 \\
7\end{array}$ & $\begin{array}{c}2 \\
4 \\
2 \\
\text { Trace }\end{array}$ & $\begin{array}{r}24 \\
18 \\
9 \\
8\end{array}$ & $\begin{array}{r}12 \\
7 \\
6 \\
5\end{array}$ & $\begin{array}{r}6 \\
10 \\
8 \\
51\end{array}$ & $\begin{array}{r}5 \\
9 \\
2 \\
11\end{array}$ \\
\hline
\end{tabular}

1 Rock types within stratigraphic units are not necessarily in order of stratigraphic sequence or abundance.

Average modes of rocks from the Mission Mountains are given in table 2, and average modes for some rocks from the Pend Oreille area have previously been published (Harrison and Campbell, 1963, table 1).

\section{ANALYTICAL TECHNIQUE AND CALCULATIONS FOR GEOCHEMISTRY}

Semiquantitative spectrographic analyses presented in this report (table 3 ) and similar analyses on rocks from the Mission Mountains (Harrison and others, 1969) were done using methods outlined by Grimes and Marranzino (1968). Analytical results were reported as approximate geometric midpoints of ranges whose boundaries are $1.2,0.83,0.56,0.38$, and 0.18 (or multiples of those numbers) ; results are reported as $1,0.7,0.5,0.3,0.2$, and 0.15 (or appropriate multiples). 
TABLE 3.-Semiquantitative spectrographic analyses

[Number in parentheses indicates sensitivity limit for method used. Metamorphic grade: C, bedding; Bgn, biotite zone in gneiss or schist. $>$, undetermined amount of element is limit; N, element was looked for but not found. Also looked for but not found in any all samples were reported as $N(5)$ for $M o, N(200)$ for $Z \mathbf{Z n} N(0.5)$ for $\mathbf{A g}$, and $N(10)$ for

\begin{tabular}{|c|c|c|c|c|c|c|c|c|c|c|c|}
\hline \multirow[b]{2}{*}{ Sample } & \multicolumn{4}{|c|}{ (percent) } & \multicolumn{6}{|c|}{$(\mathrm{ppm})$} & \\
\hline & $\begin{array}{r}\mathrm{Fe} \\
(.05) \\
\end{array}$ & $\begin{array}{c}\mathrm{Mg} \\
(.0 \mathrm{i}) \\
\end{array}$ & $\begin{array}{r}\mathrm{Ca} \\
(.05) \\
\end{array}$ & $\begin{array}{c}\mathrm{Ti} \\
(.001)\end{array}$ & $\begin{array}{c}M n \\
(10) \\
\end{array}$ & $\begin{array}{c}8 \\
(10) \\
\end{array}$ & $\begin{array}{r}\mathrm{Ba} \\
(10) \\
\end{array}$ & $\begin{array}{l}\mathrm{Be} \\
\text { (1) }\end{array}$ & $\begin{array}{l}\text { Co } \\
\text { (5) }\end{array}$ & $\begin{array}{l}c r \\
(5)\end{array}$ & $\begin{array}{c}\mathrm{La} \\
(20) \\
\end{array}$ \\
\hline
\end{tabular}

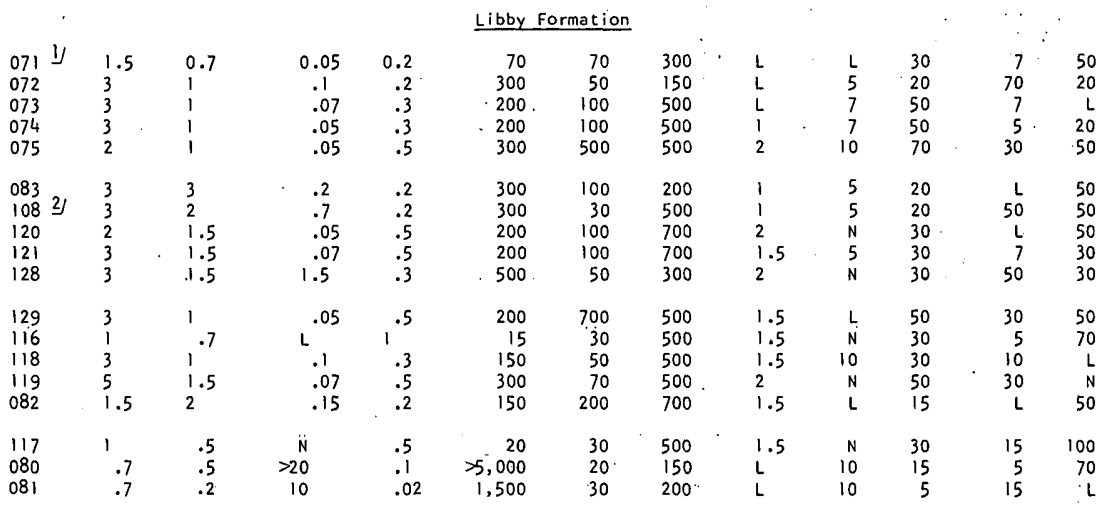

\section{Striped Peak Formation}

\begin{tabular}{|c|c|c|c|c|c|c|c|c|c|c|c|c|}
\hline $\begin{array}{l}067 \\
069 \\
097 \\
102 \\
103\end{array}$ & $\begin{array}{l}3 \\
3 \\
3 \\
3 \\
5\end{array}$ & $\begin{array}{l}1.5 \\
1.5 \\
1.5 \\
1.5 \\
1.5\end{array}$ & $\begin{array}{l}.07 \\
.07 \\
.07 \\
.07 \\
.05\end{array}$ & $\begin{array}{r}.3 \\
.5 \\
.5 \\
. .2 \\
.2\end{array}$ & $\begin{array}{r}15 \\
30 \\
150 \\
15 \\
15\end{array}$ & $\begin{array}{l}150 \\
500 \\
500 \\
300 \\
100\end{array}$ & $\begin{array}{l}700 \\
300 \\
300 \\
700 \\
700\end{array}$ & $\begin{array}{l}1.5 \\
1.5 \\
2 \\
2 \\
1.5\end{array}$ & $\begin{array}{l}\text { L } \\
5 \\
7 \\
5 \\
5\end{array}$ & $\begin{array}{r}50 \\
70 \\
70 \\
50 \\
7\end{array}$ & $\begin{array}{r}\mathrm{L} \\
20 \\
30 \\
\mathrm{~L} \\
\mathrm{~N}\end{array}$ & $\begin{array}{l}50 \\
50 \\
50 \\
50 \\
70\end{array}$ \\
\hline $\begin{array}{l}110 \\
113 \\
064 \\
055 \\
068\end{array}$ & $\begin{array}{l}3 \\
3 \\
1.5 \\
3 \\
1\end{array}$ & $\begin{array}{l}1.5 \\
1.5 \\
1.7 \\
5.5 \\
5\end{array}$ & $\begin{array}{r}.05 \\
.05 \\
.05 \\
.07\end{array}$ & $\begin{array}{l}.5 \\
.3 \\
.3 \\
.7 \\
.2\end{array}$ & $\begin{array}{r}30 \\
15 \\
15 \\
15 \\
300\end{array}$ & $\begin{array}{r}2,000 \\
100 \\
1,500 \\
300 \\
150\end{array}$ & $\begin{array}{l}300 \\
500 \\
200 \\
300 \\
300\end{array}$ & $\begin{array}{l}2 \\
1.5 \\
1 \\
1\end{array}$ & $\begin{array}{l}\mathrm{L} \\
5 \\
\mathrm{~N} \\
7 \\
\mathrm{~L}\end{array}$ & $\begin{array}{l}70 \\
70 \\
30 \\
70 \\
30\end{array}$ & $\begin{array}{r}10 \\
7 \\
5 \\
5 \\
15\end{array}$ & $\begin{array}{l}70 \\
50 \\
50 \\
70 \\
50\end{array}$ \\
\hline $\begin{array}{l}078 \\
095 \\
098 \\
105 \\
106\end{array}$ & $\begin{array}{l}1.5 \\
2 \\
1 \\
1 \\
.7\end{array}$ & $\begin{array}{l}3 \\
1.5 \\
5 \\
.3 \\
.7\end{array}$ & $\begin{array}{l}.1 \\
.07 \\
7 \\
.07 \\
.05\end{array}$ & $\begin{array}{l}.2 \\
.2 \\
.2 \\
.7 \\
.7\end{array}$ & $\begin{array}{r}50 \\
50 \\
200 \\
15 \\
20\end{array}$ & $\begin{array}{r}70 \\
50 \\
100 \\
1,500 \\
2,000\end{array}$ & $\begin{array}{l}300 \\
200 \\
700 \\
150 \\
300\end{array}$ & $\begin{array}{l}1.5 \\
L \\
L \\
2 \\
1.5\end{array}$ & $\begin{array}{l}7 \\
7 \\
5 \\
1 \\
N\end{array}$ & $\begin{array}{l}30 \\
50 \\
50 \\
30 \\
30\end{array}$ & $\begin{array}{r}\mathrm{L} \\
7 \\
20 \\
7 \\
7\end{array}$ & $\begin{array}{r}30 \\
30 \\
30 \\
.30 \\
50\end{array}$ \\
\hline $\begin{array}{l}1073 / \\
109 \\
122 \\
125 \\
062\end{array}$ & $\begin{array}{l}1 \\
3 \\
3 \\
3 \\
1\end{array}$ & $\begin{array}{l}.7 \\
3.5 \\
2 \\
.3\end{array}$ & $\begin{array}{l}.05 \\
.1 \\
.15 \\
.05\end{array}$ & $\begin{array}{l}1 \\
.3 \\
.7 \\
.5 \\
.3\end{array}$ & $\begin{array}{r}15 \\
30 \\
50 \\
70 \\
30\end{array}$ & $\begin{array}{r}2,000 \\
200 \\
200 \\
2,000 \\
50\end{array}$ & $\begin{array}{l}500 \\
300 \\
500 \\
500 \\
150\end{array}$ & $\begin{array}{l}1.5 \\
1.5 \\
1.5 \\
1.5 \\
1.5\end{array}$ & $\begin{array}{r}N \\
10 \\
7 \\
N \\
5\end{array}$ & $\begin{array}{l}50 \\
50 \\
70 \\
50 \\
20\end{array}$ & $\begin{array}{r}10 \\
5 \\
5 \\
\quad \quad 15 \\
1\end{array}$ & $\begin{array}{l}70 \\
70 \\
50 \\
50 \\
50\end{array}$ \\
\hline $\begin{array}{l}063 \\
070 \\
076 \\
100 \\
114\end{array}$ & $\begin{array}{r}2 \\
1.5 \\
.7 \\
.7 \\
.5\end{array}$ & $\begin{array}{l}1 \\
2 \\
.15 \\
.1 \\
.5\end{array}$ & $\begin{array}{l}.05 \\
1.5 \\
.07 \\
L \\
L\end{array}$ & $\begin{array}{l}.5 \\
.3 \\
.2 \\
.2 \\
.05\end{array}$ & $\begin{array}{r}100 \\
300 \\
30 \\
300 \\
70\end{array}$ & $\begin{array}{r}300 \\
150 \\
30 \\
150 \\
15\end{array}$ & $\begin{array}{l}200 \\
150 \\
100 \\
200 \\
300\end{array}$ & $\begin{array}{l}1.5 \\
1 \\
1 \\
1 \\
L\end{array}$ & $\begin{array}{r}10 \\
7 \\
N \\
\text { L } \\
\text { L }\end{array}$ & $\begin{array}{r}30 \\
30 \\
15 \\
20 \\
5\end{array}$ & $\begin{array}{r}L \\
L \\
L \\
30 \\
5\end{array}$ & $\begin{array}{r}50 \\
30 \\
20 \\
30 \\
\mathrm{~N}\end{array}$ \\
\hline $\begin{array}{l}126 \\
127 \\
211 \\
111 \\
124\end{array}$ & $\begin{array}{l}2 \\
3 \\
1.5 \\
1 \\
2\end{array}$ & $\begin{array}{l}.7 \\
.7 \\
10\end{array}$ & $\begin{array}{l}.07 \\
.1 \\
15 \\
20\end{array}$ & $\begin{array}{l}.15 \\
.5 \\
.15 \\
.1 \\
.15\end{array}$ & $\begin{array}{r}100 \\
150 \\
50 \\
500 \\
5,000\end{array}$ & $\begin{array}{r}300 \\
150 \\
30 \\
150 \\
500\end{array}$ & $\begin{array}{r}150 \\
200 \\
500 \\
70 \\
500\end{array}$ & $\begin{array}{c}1 \\
1.5 \\
L \\
300 \\
L\end{array}$ & $\begin{array}{r}L \\
15 \\
5 \\
L \\
L\end{array}$ & $\begin{array}{l}20 \\
50 \\
20 \\
20 \\
30\end{array}$ & $\begin{array}{r}15 \\
7 \\
15 \\
10 \\
3,000\end{array}$ & $\begin{array}{r}30 \\
50 \\
50 \\
N \\
30\end{array}$ \\
\hline
\end{tabular}

Wallace Formation

$\begin{array}{llllllllllllll}004 & .7 & .5 & . & L & .2 & 10 & 30 & 300 & L & 5 & 20 & L & 30 \\ 005 & 2 & 3^{.} & . .15 & .2 & 30 & 70 & 500 & 1.5 & 7 & 30 & L & 70 \\ 008 & 1.5 & 1.5 & L & .3 & 20 & 50 & 300 & L & 7 & 50 & 7 & 70 \\ 012 & 3 & 1.5 & L & .2 & 70 & 50 & 300 & 1 & 10 & 50 & 30 & 50 \\ 013 & 1.5 & 2 & L & .3 & 50 & 30 & 500 & 1 & 10 & 30 & 5 & 30\end{array}$

See footnotes at end of table. 
of Belt rocks from the Pend Oreille area

chlorite-sericite zone including minor secondary biotite; $\mathrm{Bb}$, biotite Zone in rock with prominent present above number shown: $L$, undetermined amount of element is present below sensitivity sample were $\mathrm{As}(200), \mathrm{Au}(10), \mathrm{Cd}(20), \mathrm{Sb}(100)$, and W(50). Except as indicated by footnotes, $\mathrm{Bi}$ and Sn. D. J. Grimes, analyst]

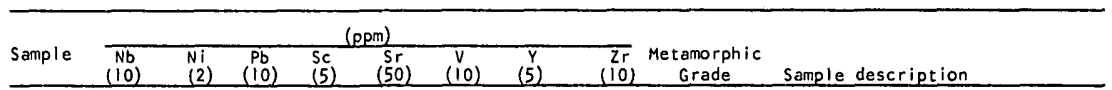

Libby Formation

\begin{tabular}{|c|c|c|c|}
\hline $\begin{array}{l}071 \\
072 \\
073 \\
074 \\
075\end{array}$ & $\begin{array}{c}L \\
L \\
L \\
L \\
10\end{array}$ & $\begin{array}{l}10 \\
15 \\
15 \\
20 \\
20\end{array}$ & $\begin{array}{r}N \\
15 \\
L \\
L \\
L\end{array}$ \\
\hline $\begin{array}{l}083 \\
108 \\
120 \\
121 \\
128\end{array}$ & $\begin{array}{r}L \\
L \\
10 \\
10 \\
10\end{array}$ & $\begin{array}{r}30 \\
15 \\
L \\
20 \\
20\end{array}$ & $\begin{array}{r}N \\
N \\
N \\
10 \\
L\end{array}$ \\
\hline $\begin{array}{l}129 \\
116 \\
118 \\
119 \\
082\end{array}$ & $\begin{array}{r}15 \\
15 \\
L \\
10 \\
L\end{array}$ & $\begin{array}{r}20 \\
\text { L } \\
20 \\
30 \\
5\end{array}$ & $\begin{array}{l}N \\
L \\
N \\
N \\
N\end{array}$ \\
\hline $\begin{array}{l}117 \\
080 \\
081\end{array}$ & $\begin{array}{r}10 \\
\mathrm{~N} \\
\mathrm{~N}\end{array}$ & $\begin{array}{l}L \\
L \\
5\end{array}$ & $\begin{array}{l}30 \\
30 \\
30\end{array}$ \\
\hline
\end{tabular}

067
069
097
102
103
110
113
064
065
068
078
095
098
105
106
107
109
122
125
062
063
063
070
076
100
114
11
126
127
211
111
124

004
005
008
012
013

100

\section{Striped Peak Formation}

\begin{tabular}{|c|c|c|}
\hline $\begin{array}{l}70 \\
70 \\
70 \\
70 \\
50\end{array}$ & $\begin{array}{l}30 \\
30 \\
30 \\
50 \\
50\end{array}$ & $\begin{array}{l}200 \\
150 \\
150 \\
200 \\
150\end{array}$ \\
\hline $\begin{array}{l}70 \\
70 \\
30 \\
70 \\
30\end{array}$ & $\begin{array}{l}30 \\
30 \\
30 \\
50 \\
15\end{array}$ & $\begin{array}{r}200 \\
200 \\
700 \\
1,000 \\
200\end{array}$ \\
\hline $\begin{array}{l}50 \\
30 \\
50 \\
70 \\
70\end{array}$ & $\begin{array}{l}30 \\
30 \\
20 \\
30 \\
30\end{array}$ & $\begin{array}{l}150 \\
200 \\
150 \\
200 \\
200\end{array}$ \\
\hline $\begin{array}{l}70 \\
70 \\
70 \\
70 \\
30\end{array}$ & $\begin{array}{l}50 \\
30 \\
30 \\
30 \\
20\end{array}$ & $\begin{array}{l}300 \\
150 \\
700 \\
300 \\
700\end{array}$ \\
\hline $\begin{array}{l}50 \\
70 \\
15 \\
50 \\
20\end{array}$ & $\begin{array}{l}20 \\
30 \\
15 \\
20 \\
10\end{array}$ & $\begin{array}{l}700 \\
300 \\
700 \\
200 \\
100\end{array}$ \\
\hline $\begin{array}{l}70 \\
70 \\
50 \\
20 \\
50\end{array}$ & $\begin{array}{l}20 \\
30 \\
20 \\
10 \\
30\end{array}$ & $\begin{array}{r}300 \\
1,000 \\
200 \\
20 \\
150\end{array}$ \\
\hline \multicolumn{3}{|c|}{ Wallace Formation } \\
\hline $\begin{array}{l}50 \\
50 \\
70 \\
50 \\
50\end{array}$ & $\begin{array}{l}20 \\
30 \\
20 \\
20 \\
15\end{array}$ & $\begin{array}{l}150 \\
100 \\
100 \\
100 \\
100\end{array}$ \\
\hline
\end{tabular}

Black silty argillite. Calcareous silty argillite. Black silty argillite. Green silty argillite. Black silty argillite.

Green silty argillite. Calcareous green argillite. Black silty argillite.

$$
\text { Do. }
$$

Green calcareous argillite.

Black silty argillite. Do.

Do.

Green argillitic siltite.

Argillitic siltite.

Stromatolite.

Cherty oolite.

Green silty argillite. Black silty argillite. Do.

Green silty argillite. Maroon argillite.

Black silty argillite. Red silty argillite.

Olive siltite.

Red siltite with salt casts. Dolomitic siltite.

Dolomitic argillitic siltite. Red and green siltite.

Dolomitic siltite.

Green siltite.

Black argillitic siltite.

Do.

Red feldspathic siltite.

Green argillitic siltite.

Black argillitic siltite.

Red feldspathic quartzite.

Green quartzite.

Calcareous red quartzite.

Red feldspathic quartzite.

Do.

$$
\text { Do. }
$$

Green quartzite.

Red feldspathic quartzite.

Green quartzite.

Silty dolomite.

Argillitic dolomite.

Black silty argillite.

Waxy green argillite.

Green silty argillite.

black silty argillite.

Waxy green argillite. 
$\mathrm{T}_{\mathrm{ABLE}}$ 3.-Semiquantitative spectrographic analyses

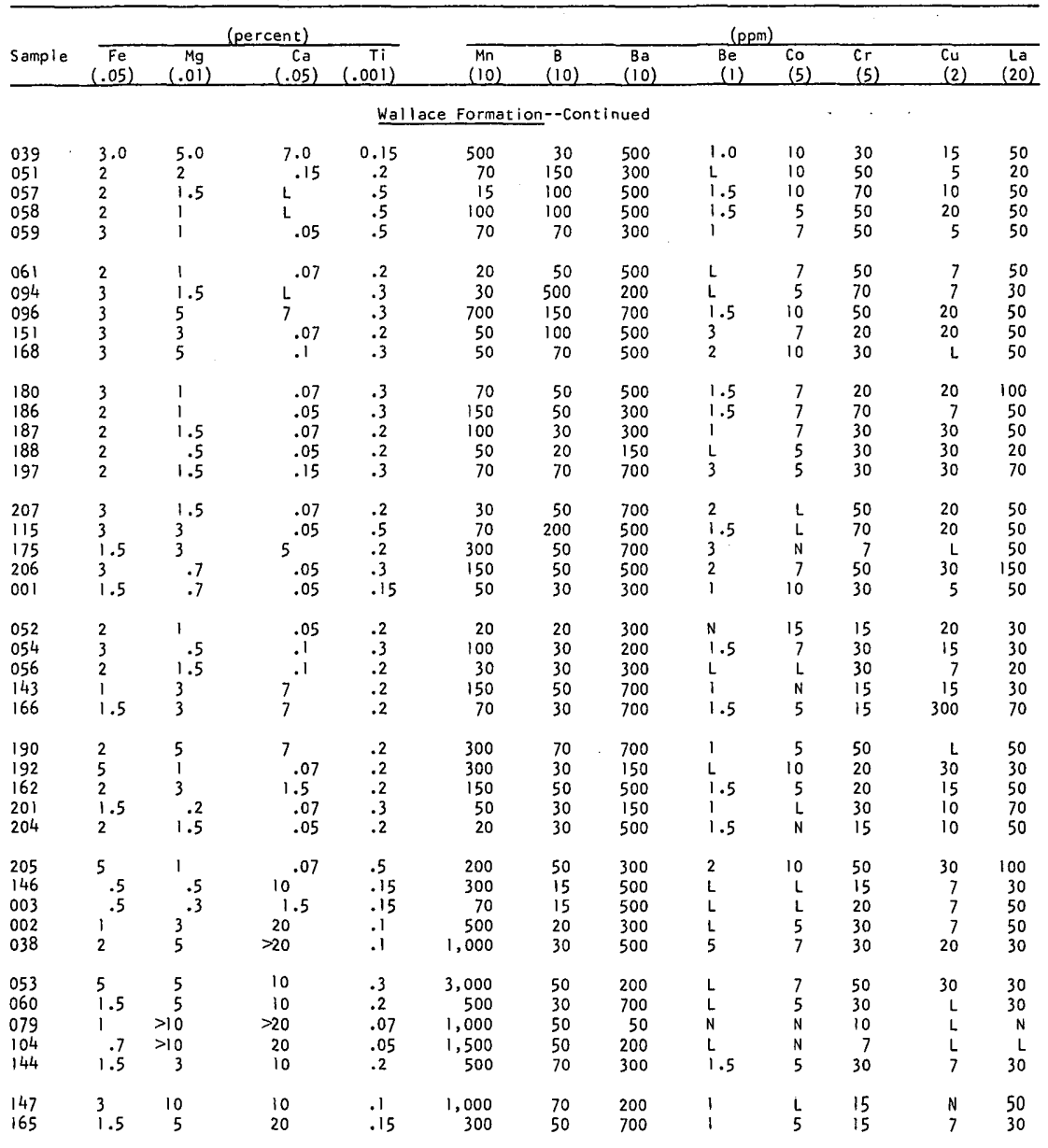

\section{St. Regis Formation}

$\begin{array}{rllllrrrrrrrr}006 & 3 & 2 & .07 & .2 & 70 & 30 & 700 & \mathrm{~L} & 10 & 50 & \mathrm{~L} & 70 \\ 046 & 3 & 1.5 & 2 & .2 & 1,000 & 30 & 700 & 1.5 & 7 & 50 & \mathrm{~L} & 50 \\ 152 & 3 & 2 & .1 & .5 & 50 & 70 & 500 & 1.5 & 7 & 20 & \mathrm{~L} & 50 \\ 169 & 3 & 2 & .07 & .5 & 30 & 100 & 500 & 2 & 5 & 50 & 10 & 70 \\ 193 & 2 & .5 & .05 & .2 & 30 & 30 & 300 & 1 & 7 & 50 & 10 & 50 \\ 195 & 3 & 1.5 & .5 & .3 & 200 & 70 & 700 & 2 & 7 & 50 & \mathrm{~L} & 70 \\ 176 & 3 & 1.5 & .1 & .5 & 300 & 70 & 700 & 3 & 10 & 30 & \mathrm{~L} & 70 \\ 007 & .7 & .5 & .15 & .15 & 100 & 15 & 300 & \mathrm{~N} & \mathrm{~L} & 15 & \mathrm{~L} & 20 \\ 084 & 3 & 3 & .1 & .3 & 300 & 70 & 700 & 1.5 & 10 & 50 & 30 & 50 \\ 153 & 3 & .5 & .2 & .3 & 300 & 30 & 500 & 1 & \mathrm{~L} & 20 & 20 & \mathrm{~L}\end{array}$

See footnotes at end of table. 
of Belt rocks from the Pend Oreille area-Continued

\begin{tabular}{|c|c|c|c|c|c|c|c|c|c|c|}
\hline \multirow{2}{*}{ Sample } & \multicolumn{9}{|c|}{$(\mathrm{ppm})$} & \multirow[b]{2}{*}{ Sample description } \\
\hline & $\begin{array}{l}\text { Nb } \\
\text { (10) }\end{array}$ & $\begin{array}{l}\mathrm{Ni} \\
\text { (2) } \\
\end{array}$ & $\begin{array}{l}\mathrm{Pb} \\
\text { (10) }\end{array}$ & $\begin{array}{r}5 c \\
-(5) \\
\end{array}$ & $\begin{array}{r}5 r \\
(50) \\
\end{array}$ & $\begin{array}{l}V \\
(10)\end{array}$ & $\begin{array}{l}Y \\
(5) \\
\end{array}$ & \multicolumn{2}{|c|}{ Tr Metamorphic } & \\
\hline \multicolumn{11}{|c|}{ Wallace Formation--Cont inued } \\
\hline 039 & L & 20 & 10 & 7 & $\mathrm{~N}$ & 50 & 20 & 150 & c & \multirow{10}{*}{$\begin{array}{l}\text { Dolomitic silty argillite. } \\
\text { Black silty argillite. } \\
\text { Do. } \\
\text { Do. } \\
\text { Do. } \\
\text { Do. } \\
\text { Do. } \\
\text { Dolomitic silty argillite. } \\
\text { Waxy green argillite. } \\
\text { Do. }\end{array}$} \\
\hline 051 & $\mathrm{~N}$ & 30 & 20 & 10 & $\mathrm{~N}$ & 50 & 15 & 150 & $\mathrm{c}$ & \\
\hline 057 & L & 30 & 15 & 10 & $\mathrm{~N}$ & 70 & 30 & 200 & c & \\
\hline 058 & 10 & 30 & 15 & 10 & N & 70 & 30 & 200 & c & \\
\hline 059 & $L$ & 30 & $\mathrm{~N}$ & 7 & N & 70 & 50 & 300 & c & \\
\hline 061 & L & 30 & $\mathrm{~N}$ & 7 & $\mathrm{~N}$ & 50 & 30 & 150 & c & \\
\hline 094 & L & 20 & 15 & 10 & $\mathrm{~N}$ & 70 & 30 & 150 & c & \\
\hline 096 & $\mathrm{~L}$ & 20 & $\mathrm{~N}$ & 7 & $\mathrm{~N}$ & 70 & 30 & 150 & c & \\
\hline 151 & 10 & 20 & $\mathrm{~N}$ & 10 & N & 70 & 30 & 150 & c & \\
\hline 168 & L & 20 & $\mathrm{~N}$ & 10 & N & 70 & 30 & 150 & c & \\
\hline 180 & 10 & 30 & $\mathrm{~L}$ & 10 & N & 70 & 30 & 150 & c & \multirow{5}{*}{$\begin{array}{l}\text { Black silty argillite. } \\
\text { Do. } \\
\text { Do. } \\
\text { Do. } \\
\text { Waxy green argillite. }\end{array}$} \\
\hline 186 & 10 & 30 & N & 10 & $\mathrm{~N}$ & 70 & 30 & 150 & c & \\
\hline 187 & L & 20 & 20 & 10 & $\mathrm{~N}$ & 70 & 30 & 150 & c & \\
\hline 188 & 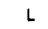 & 20 & $\mathrm{~N}$ & 7 & $\mathrm{~N}$ & 70 & 20 & 150 & c & \\
\hline 197 & 10 & 20 & 10 & 15 & $\mathrm{~N}$ & 70 & 30 & 150 & C & \\
\hline 207 & 10 & 30 & 10 & 10 & $\mathrm{~N}$ & 70 & 30 & 150 & c & \multirow{5}{*}{$\begin{array}{l}\text { 8lack silty argillite. } \\
\text { Do. } \\
\text { Dolomitic silty argillite. } \\
\text { Black silty argillite. } \\
\text { Argillitic siltite. }\end{array}$} \\
\hline 115 & 10 & 10 & $\mathrm{~L}$ & 10 & $\mathrm{~N}$ & 70 & 30 & 200 & $\mathrm{Bb}$ & \\
\hline 175 & 15 & 5 & $N$ & 7 & $\mathrm{~N}$ & 50 & 50 & 150 & $\mathrm{Bb}$ & \\
\hline 206 & 10 & 20 & $\mathrm{~N}$ & 10 & $\mathrm{~N}$ & 70 & 30 & 150 & $B D$ & \\
\hline 001 & $\mathrm{~N}$ & 30 & $L$ & 7 & $\mathrm{~N}$ & 50 & 30 & 150 & C & \\
\hline 052 & $\mathrm{~N}$ & 30 & 15 & L & N & 20 & 15 & 100 & c & \multirow{5}{*}{$\begin{array}{l}\text { White siltite. } \\
\text { Argillitic siltite. } \\
\text { Do. } \\
\text { Calcareous siltite. } \\
\text { Dolomitic argillitic siltite. }\end{array}$} \\
\hline 054 & L & 30 & N & 10 & N & 70 & 30 & 200 & C & \\
\hline 056 & $L$ & 30 & $\mathrm{~N}$ & 7 & N & 50 & 20 & 150 & C & \\
\hline 143 & L & 15 & 10 & 7 & $\mathrm{~N}$ & 50 & 20 & 150 & C & \\
\hline 166 & $\dot{L}$ & 20 & $\mathrm{~N}$ & 7 & N & 50 & 30 & 150 & c & \\
\hline 190 & $\mathrm{~L}$ & 20 & $\mathrm{~N}$ & 10 & $\mathrm{~N}$ & 70 & 30 & 100 & c & \multirow{5}{*}{$\begin{array}{l}\text { Do. } \\
\text { Argillitic siltite. } \\
\text { Calcareous siltite. } \\
\text { Green siltite. } \\
\text { Do. }\end{array}$} \\
\hline 192 & 10 & 30 & $\mathrm{~L}$ & 7 & $\mathrm{~N}$ & 50 & 30 & 150 & c & \\
\hline 162 & $L$ & 30 & L & 7 & $N$ & 70 & 30 & 150 & $B b$ & \\
\hline 201 & L & 10 & 20 & 10 & $\mathrm{~N}$ & 50 & 30 & 300 & $B b$ & \\
\hline 204 & 10 & 20 & 10 & 7 & $\mathrm{~N}$ & 50 & 20 & 150 & Bb & \\
\hline 205 & 10 & 30 & $L$ & 15 & $\mathrm{~N}$ & 70 & 30 & 150 & $8 b$ & \multirow{5}{*}{$\begin{array}{l}\text { Do. } \\
\text { Calcareous silty quartzite. } \\
\text { Calcareous quartzite. } \\
\text { Molar tooth limestone. } \\
\text { Do. }\end{array}$} \\
\hline 146 & $\mathrm{~L}$ & $\mathrm{~L}$ & 10 & L & 100 & 20 & 30 & 100 & C & \\
\hline 003 & $\mathrm{~N}$ & $L$ & $\mathrm{~L}$ & 5 & $\mathrm{~N}$ & 20 & 10 & 150 & $B b$ & \\
\hline 002 & $\mathrm{~N}$ & 15 & $\mathrm{~N}$ & 7 & 150 & 30 & 30 & 50 & $c$ & \\
\hline 038 & $\ddot{L}$ & 15 & $\mathrm{~N}$ & 5 & 300 & 30 & 20 & 30 & c & \\
\hline 053 & $\mathrm{~N}$ & 20 & L & 7 & $\mathrm{~N}$ & 50 & 30 & 200 & c & \multirow{5}{*}{$\begin{array}{l}\text { Stromatolite. } \\
\text { Silty dolomite. } \\
\text { Do. } \\
\text { Stromatolite. } \\
\text { Molar tooth I imestone. }\end{array}$} \\
\hline 060 & L & 20 & $\mathrm{~N}$ & 5 & 100 & 30 & 20 & 100 & c & \\
\hline 079 & $\mathrm{~N}$ & $L$ & 15 & $\hat{L}$ & N & 30 & 15 & 30 & C & \\
\hline 104 & N & $\mathrm{L}$ & $\mathrm{N}$ & $\mathrm{N}$ & N & 10 & 10 & 50 & c & \\
\hline 144 & L & 15 & 70 & 7 & L & 70 & 20 & 150 & C & \\
\hline 147 & L & 15 & $\mathrm{~N}$ & 5 & 100 & 50 & 30 & 50 & c & \multirow{2}{*}{$\begin{array}{l}\text { Argillitic dolomite. } \\
\text { Molar tooth limestone. }\end{array}$} \\
\hline 165 & $\mathrm{~N}$ & 15 & 50 & 5 & 150 & 50 & 20 & 50 & C & \\
\hline \multicolumn{11}{|c|}{ St. Regis Formation } \\
\hline 006 & L & 15 & $\mathrm{~N}$ & 7 & N & 50 & 30 & 100 & c & \multirow{5}{*}{$\begin{array}{l}\text { Purple argillite. } \\
\text { Do. } \\
\text { Do. } \\
\text { Do. } \\
\text { Co. }\end{array}$} \\
\hline 046 & 10 & 20 & $\ddot{L}$ & 7 & $\mathrm{~N}$ & 70 & 30 & 150 & $c$ & \\
\hline 152 & L & 20 & N & 10 & N & 70 & 30 & 150 & c & \\
\hline 169 & $\bar{L}$ & 20 & N & 10 & N & 70 & 30 & 150 & c & \\
\hline 193 & $\bar{L}$ & 20 & N & 7 & $\mathrm{~N}$ & 70 & 30 & 150 & C & \\
\hline 195 & 10 & 30 & $N$ & 10 & N & 70 & 30 & 150 & c & Purple silty argillite. \\
\hline 176 & 15 & 30 & ᄂ & 10 & $\mathrm{~N}$ & 70 & 50 & 200 & $B b$ & \\
\hline 007 & $\mathrm{~N}$ & 10 & $\mathrm{~N}$ & $\mathrm{~L}$ & $\mathrm{~N}$ & 20 & 10 & 70 & c & Purple argillitic siltite. \\
\hline 084 & $\mathrm{~L}$ & 20 & 30 & 10 & $\mathrm{~N}$ & 70 & 30 & 200 & c & Green argillitic siltite. \\
\hline 153 & 10 & 10 & L & 7 & $\mathrm{~N}$ & 50 & 30 & 200 & c & Purple argillitic siltite. \\
\hline
\end{tabular}


TABLE 3.-Semiquantitative spectrographic analyses

\begin{tabular}{|c|c|c|c|c|c|c|c|c|c|c|}
\hline \multirow[b]{2}{*}{ Sample } & \multicolumn{2}{|c|}{ (percent) } & \multicolumn{8}{|c|}{ (ppm) } \\
\hline & $\begin{array}{cc}\mathrm{Fe} & \mathrm{Mg} \\
(.05) & (.01) \\
\end{array}$ & $\begin{array}{cc}\mathrm{ca} & \mathrm{Ti} \\
(.05) & (.001) \\
\end{array}$ & $\begin{array}{l}\mathrm{Mn} \\
(10) \\
\end{array}$ & $\begin{array}{c}B \\
(10)\end{array}$ & $\begin{array}{c}B a \\
(10) \\
\end{array}$ & $\begin{array}{l}\mathrm{Be} \\
\text { (1) }\end{array}$ & $\begin{array}{l}\text { Co } \\
(5) \\
\end{array}$ & 8 & 5) & $\begin{array}{r}\mathrm{La} \\
(20) \\
\end{array}$ \\
\hline
\end{tabular}

St. Regis formation--Continued

$\begin{array}{rlllrrrrrrrrr}170 & 1.5 & 1.5 & 1.5 & 0.2 & 200 & 30 & 300 & 1.0 & \text { L } & 15 & 7 & 50 \\ 194 & 1.5 & 1.5 & 2 & .2 & 1,000 & 30 & 300 & 1 & 5 & 20 & 10 & 50 \\ 196 & 2 & 1.5 & .07 & .2 & 50 & 30 & 300 & 1.5 & 7 & 15 & 20 & 50 \\ 047 & 1.5 & 2 & 1.5 & .2 & 300 & 30 & 300 & 1 & 7 & 30 & 15 & 50 \\ 163 & 5 & 1.5 & .15 & .3 & 50 & 50 & 500 & 2 & 7 & 50 & 5 & 100 \\ 184 & 1.5 & 3 & .1 & .3 & 100 & 200 & 150 & 1.5 & 5 & 15 & L & 50\end{array}$

\section{Revett Formation}

\begin{tabular}{|c|c|c|c|c|c|c|c|c|c|c|c|}
\hline $\begin{array}{l}009 \\
043 \\
048 \\
171 \\
091\end{array}$ & $\begin{array}{l}1.5 \\
1 \\
1 \\
3 \\
1\end{array}$ & $\begin{array}{l}1 \\
.15 \\
.3 \\
.3 \\
.3\end{array}$ & $\begin{array}{l}.07 \\
L^{.07} \\
.07 \\
.05\end{array}$ & $\begin{array}{l}.2 \\
.5 \\
.3 \\
.3 \\
.2\end{array}$ & $\begin{array}{r}70 \\
50 \\
20 \\
20 \\
200\end{array}$ & $\begin{array}{r}20 \\
100 \\
30 \\
20 \\
15\end{array}$ & $\begin{array}{l}300 \\
300 \\
300 \\
500 \\
300\end{array}$ & $\begin{array}{l}1 \\
1 \\
1.5 \\
2 \\
1.5\end{array}$ & $\begin{array}{r}10 \\
L \\
L \\
L \\
L\end{array}$ & $\begin{array}{l}30 \\
30 \\
30 \\
20 \\
15\end{array}$ & $\begin{array}{l}\mathrm{L} \\
\mathrm{L} \\
5 \\
\mathrm{~L} \\
7\end{array}$ \\
\hline $\begin{array}{l}174 \\
181 \\
010 \\
042 \\
050\end{array}$ & $\begin{array}{l}2 \\
.7 \\
.7 \\
.7\end{array}$ & $\begin{array}{l}.7 \\
.3 \\
.07 \\
.05 \\
.07\end{array}$ & $\begin{array}{l}L_{\mathrm{N}}^{.05} \\
\mathrm{~L}\end{array}$ & $\begin{array}{l}.5 \\
.3 \\
.2 \\
.3 \\
.1\end{array}$ & $\begin{array}{r}15 \\
20 \\
50 \\
100 \\
700\end{array}$ & $\begin{array}{r}30 \\
L \\
15 \\
30 \\
15\end{array}$ & $\begin{array}{l}700 \\
150 \\
300 \\
150 \\
150\end{array}$ & $\begin{array}{l}3 \\
1 \\
L \\
1.5 \\
L\end{array}$ & $\begin{array}{l}\mathrm{N} \\
\mathrm{N} \\
\mathrm{N} \\
\mathrm{N} \\
\mathrm{L}\end{array}$ & $\begin{array}{r}30 \\
7 \\
15 \\
20 \\
7\end{array}$ & $\begin{array}{r}\mathrm{L} \\
\mathrm{N} \\
5 \\
\mathrm{~L} \\
30\end{array}$ \\
\hline $\begin{array}{l}090 \\
203 \\
087 \\
158 \\
173\end{array}$ & $\begin{array}{r}.3 \\
.3 \\
.5 \\
.5\end{array}$ & $\begin{array}{l}.07 \\
.1 \\
.3 \\
.07 \\
.1\end{array}$ & $\begin{array}{l}L^{L} \\
.05 \\
.05\end{array}$ & $\begin{array}{l}.15 \\
.05 \\
.15 \\
.15 \\
.15\end{array}$ & $\begin{array}{l}30 \\
15 \\
70 \\
20 \\
50\end{array}$ & $\begin{array}{r}10 \\
L \\
10 \\
L \\
10\end{array}$ & $\begin{array}{l}300 \\
200 \\
300 \\
150 \\
200\end{array}$ & $\begin{array}{l}\mathrm{L} \\
\mathrm{L} \\
\mathrm{I} \\
\mathrm{L} \\
\mathrm{I}\end{array}$ & $\begin{array}{l}\mathrm{N} \\
\mathrm{N} \\
7 \\
\mathrm{~N} \\
\mathrm{~N}\end{array}$ & $\begin{array}{l}5 \\
7 \\
7 \\
5 \\
5\end{array}$ & $\begin{array}{l}\mathrm{L} \\
\mathrm{L} \\
5 \\
5 \\
5\end{array}$ \\
\hline $\begin{array}{l}182 \\
200\end{array}$ & $\begin{array}{r}.3 \\
.2\end{array}$ & $\begin{array}{r}.15 \\
.05\end{array}$ & $\frac{\mathrm{L}}{\mathrm{N}}$ & $\begin{array}{l}.2 \\
.15\end{array}$ & $\begin{array}{l}30 \\
20\end{array}$ & $\begin{array}{l}10 \\
10\end{array}$ & $\begin{array}{l}150 \\
300\end{array}$ & 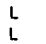 & $\begin{array}{l}\mathrm{N} \\
\mathrm{N}\end{array}$ & $\begin{array}{l}5 \\
5\end{array}$ & $\begin{array}{r}5 \\
15\end{array}$ \\
\hline
\end{tabular}

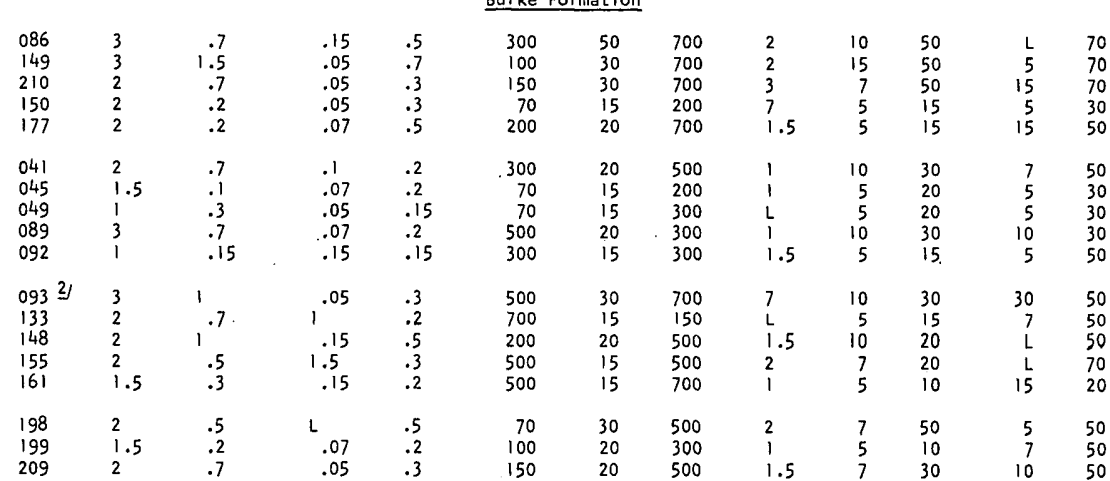

\section{Prichard Formation}

\begin{tabular}{|c|c|c|c|c|c|c|c|c|c|c|c|}
\hline $\begin{array}{l}040 \\
131 \\
183 \\
0175 \\
020\end{array}$ & $\begin{array}{l}3 \\
3 \\
1.5 \\
3 \\
3\end{array}$ & $\begin{array}{r}.7 \\
.3 \\
.7\end{array}$ & $\begin{array}{r}.05 \\
.05 \\
.15 \\
.05\end{array}$ & $\begin{array}{l}.2 \\
.5 \\
.2 \\
.3 \\
.5\end{array}$ & $\begin{array}{r}300 \\
300 \\
15 \\
300 \\
300\end{array}$ & $\begin{array}{l}30 \\
50 \\
20 \\
15 \\
30\end{array}$ & $\begin{array}{l}500 \\
700 \\
500 \\
300 \\
700\end{array}$ & $\begin{array}{l}1.5 \\
10^{1.5} \\
3 \\
1 \\
1.5\end{array}$ & $\begin{array}{r}L \\
N \\
5 \\
20 \\
15\end{array}$ & $\begin{array}{r}30 \\
70 \\
7 \\
50 \\
70\end{array}$ & $\begin{array}{r}5 \\
10 \\
20 \\
50 \\
7\end{array}$ \\
\hline $\begin{array}{l}021 \\
023 \\
026 \\
031 \\
034\end{array}$ & $\begin{array}{l}2 \\
1.5 \\
2 \\
3 \\
3\end{array}$ & $\begin{array}{l}1^{.7} \\
1 \\
1.5\end{array}$ & $\begin{array}{l}.05 \\
.2 \\
.07 \\
.07 \\
.7\end{array}$ & $\begin{array}{l}.3 \\
.2 \\
.3 \\
.3 \\
.3\end{array}$ & $\begin{array}{l}300 \\
200 \\
200 \\
300 \\
500\end{array}$ & $\begin{array}{l}20 \\
10 \\
30 \\
15 \\
15\end{array}$ & $\begin{array}{l}500 \\
500 \\
700 \\
700 \\
500\end{array}$ & $\begin{array}{l}1 \\
L \\
1.5 \\
1 \\
1.5\end{array}$ & $\begin{array}{l}7 \\
7 \\
7 \\
7 \\
5\end{array}$ & $\begin{array}{l}30 \\
30 \\
30 \\
50 \\
70\end{array}$ & $\begin{array}{r}20 \\
10 \\
5 \\
L \\
7\end{array}$ \\
\hline
\end{tabular}

See footnotes at end of table. 


\begin{tabular}{|c|c|c|c|c|c|c|c|c|c|}
\hline \multirow[b]{2}{*}{ Sample } & \multicolumn{6}{|c|}{$(\mathrm{ppm})$} & \multirow{2}{*}{\multicolumn{2}{|c|}{$\begin{array}{l}\text { 2r Metamorphic } \\
\text { (10) Grade }\end{array}$}} & \\
\hline & $\begin{array}{l}\mathrm{Nb} \\
(10)\end{array}$ & $\begin{array}{cc}\mathrm{Ni} & \mathrm{Pb} \\
(2) & (10)\end{array}$ & $\begin{array}{l}\mathrm{Sc} \\
\text { (5) }\end{array}$ & $\begin{array}{c}5 r \\
(50)\end{array}$ & $\begin{array}{l}v \\
(10)\end{array}$ & $\begin{array}{l}Y \\
(5)\end{array}$ & & & Sample description \\
\hline
\end{tabular}

\section{St. Regis Formation--Continued}

$\begin{array}{rrrrr}170 & \mathrm{~L} & 10 & \mathrm{~N} & 5 \\ 194 & \mathrm{~L} & 20 & \mathrm{~N} & 7 \\ 196 & 10 & 20 & \mathrm{~N} & 7 \\ 047 & \mathrm{~L} & 15 & \mathrm{~N} & 7 \\ 163 & 10 & 30 & \mathrm{~N} & 15 \\ 184 & \mathrm{~L} & 20 & \mathrm{~N} & 7\end{array}$

$\begin{array}{rr}50 & 30 \\ 50 & 30 \\ 50 & 20 \\ 30 & 30 \\ 100 & 50\end{array}$

$\begin{array}{ll}30 & 150 \\ 30 & 150\end{array}$

150
150

150

200

70

\section{Revett Formation}

009
04
048
171
09
174
181
010
042
050
090
203
08
158
173
182
200

009
043
048
171
091
174
181
010
042
050
090
203
087
158
173
182
200

043

086
149
210
150
177
041
045
049
089
092
093
133
148
155
161
198
199
209

092

093

133
148

155
161

198

199

L
10
L
10
L
10
10
$N$
L
$N$
L
$N$
$N$
L
L
L
L

$\begin{array}{rrr}15 & \mathrm{~N} & 7 \\ 7 & \mathrm{~N} & 7 \\ 5 & \mathrm{~N} & 10 \\ 20 & \mathrm{~N} & 7 \\ 5 & \mathrm{~N} & 5\end{array}$

,

$\mathrm{N}$
$\mathrm{N}$
$\mathrm{N}$
$\mathrm{N}$
$\mathrm{N}$
$\mathrm{N}$
$\mathrm{N}$
$\mathrm{N}$
$\mathrm{N}$
$\mathrm{N}$
$\mathrm{N}$
$\mathrm{N}$
$\mathrm{N}$
$\mathrm{N}$
$\mathrm{N}$
$\mathrm{N}$
$\mathrm{N}$

$\begin{array}{rrr}50 & 15 & 100 \\ 50 & 30 & 200 \\ 30 & 50 & 1,000 \\ 70 & 30 & 300 \\ 20 & 20 & 150 \\ 70 & 30 & 700 \\ 30 & 20 & 1,000 \\ 10 & 15 & 300 \\ 20 & 20 & >1,000 \\ 10 & 20 & 300 \\ 10 & 10 & 150 \\ \mathrm{~L} & 15 & 100 \\ 30 & 15 & 150 \\ 30 & 30 & 500 \\ 30 & 20 & 700 \\ 20 & 20 & 200 \\ 15 & 20 & 1,000\end{array}$

Burke Formation

$\begin{array}{lll}70 & 30 & 150\end{array}$

$\begin{array}{lll}70 & 30 & 150 \\ 70 & 50 & 300 \\ 70 & 30 & 150 \\ 50 & 30 & 150\end{array}$

$\begin{array}{lll}50 & 30 & 150\end{array}$

$70 \quad 30 \quad 150$

$30 \quad 30 \quad 150$

$\begin{array}{lll}30 & 20 & 150 \\ 20 & 20 & 200\end{array}$

$\begin{array}{lll}30 & 15 & 150\end{array}$

$30 \quad 15$

$\begin{array}{lll}70 & 30 & 150 \\ 50 & 30 & 200\end{array}$

$\begin{array}{lll}70 & 30 & 300\end{array}$

$50 \quad 30 \quad 200$

$50 \quad 30 \quad 150$

$50 \quad 20 \quad 150$

$\begin{array}{lll}50 & 20 & 150 \\ 50 & 30 & 150\end{array}$

$\begin{array}{rrrrr}040 & 10 & \mathrm{~L} & 10 & 7 \\ 131 & 10 & \mathrm{~L} & 30 & 15 \\ 183 & \mathrm{~L} & 15 & \mathrm{~N} & 5 \\ 017 & \mathrm{~L} & 30 & 20 & 7 \\ 020 & 15 & 20 & \mathrm{~L} & 15 \\ 021 & \mathrm{~L} & 10 & 15 & 7 \\ 023 & \mathrm{~L} & 20 & 15 & 7 \\ 026 & 15 & 15 & 15 & 10 \\ 031 & \mathrm{~L} & 15 & 10 & 15 \\ 034 & \mathrm{~L} & 7 & 15 & 10\end{array}$

\section{Prichard Formation}

$\begin{array}{rrr}50 & 20 & 150 \\ 100 & 30 & 150 \\ 30 & 30 & 150 \\ 50 & 15 & 100 \\ 100 & 30 & 150 \\ 70 & 30 & 100 \\ 50 & 15 & 70 \\ 50 & 30 & 150 \\ 70 & 30 & 100 \\ 70 & 30 & 150\end{array}$

Dolomitic purple siltite. Do.

Purple argillitic siltite.

Dolomitic purple siltite.

Purple argillitic siltite,

Bb Waxy green argillitic siltite.

Green siltite.

Do.

Do.

Do.

Purple streaked quartzite.

White quartzite.

Calcareous quartzite.

White quartzite.

Do.

Do.

Do.

Purple streaked quartzite.

White quartzite.

Do.

Gray silty argillite

Do.

Do.

Purple streaked siltite.

Gray argillitic siltite.

Do.

Purple streaked siltite.

Gray argillitic siltite.

Do.

Purple streaked siltite.

Gray-green argillitic siltite. Gray argllitic siltite.

Do.

Purple and green siltite.

Purple argilitic siltite.

Do.

Gray-green argillitic siltite.

Black and white argillite.

Do.

Gray pyrrhotitic argllite.

Do.

Black and white argillite. Pyrrhotitic sllty argillite.

Gray silty argillite.

Spotted silty argillite. Do. 
TABLE 3.-Semiquantitative spectrographic analyses

\begin{tabular}{|c|c|c|c|c|c|c|c|c|c|c|c|c|}
\hline \multirow{2}{*}{ Sample } & \multicolumn{4}{|c|}{ (percent) } & \multicolumn{8}{|c|}{ (ppm) } \\
\hline & $\begin{array}{l}\mathrm{Fe} \\
(.05) \\
\end{array}$ & $\begin{array}{r}\mathrm{Mg} \\
(.01) \\
\end{array}$ & $\begin{array}{c}c a \\
(.05) \\
\end{array}$ & $\begin{array}{r}T i \\
(.001) \\
\end{array}$ & $\begin{array}{l}\overline{M n} \\
(10)\end{array}$ & $\begin{array}{c}8 \\
(10) \\
\end{array}$ & $\begin{array}{c}B a \\
(10)\end{array}$ & $\begin{array}{l}\mathrm{Be} \\
\text { (1) }\end{array}$ & $\begin{array}{l}\text { Co } \\
\text { (5) }\end{array}$ & $\begin{array}{l}\mathrm{Cr} \\
(5) \\
\end{array}$ & $\begin{array}{l}\mathrm{Cu} \\
\text { (2) }\end{array}$ & $\begin{array}{r}\text { La } \\
(20) \\
\end{array}$ \\
\hline \multicolumn{13}{|c|}{ Prichard Formation--Cont inued } \\
\hline 035 & 3.0 & 1.0 & 0.2 & 0.5 & 300 & 15 & 700 & L & 10 & 70 & L & 70 \\
\hline 037 & 3 & .7 & L & .2 & 200 & 15 & 700 & $i$ & 7 & 30 & 7 & 30 \\
\hline 085 & 3 & .7 & .05 & .3 & 300 & 20 & 500 & $i$ & 10 & 30 & 20 & 50 \\
\hline 132 & 3 & $1 "$ & .15 & .5 & 500 & 50 & 700 & 2 & 15 & 70 & 10 & 70 \\
\hline 135 & 3 & .7 & .3 & .5 & 500 & 30 & 700 & 3 & 10 & 50 & 10 & 50 \\
\hline 136 & 5 & .7 & .07 & .5 & 300 & 30 & 700 & 2 & 5 & 50 & 20 & 30 \\
\hline 137 & 3 & .7 & .15 & .5 & 500 & 30 & 700 & 2 & 10 & 30 & 5 & 50 \\
\hline 138 & 2 & .5 & .07 & .3 & 300 & 30 & 500 & 2 & L & 20 & 7 & 30 \\
\hline 139 & 3 & 1 & .07 & .5 & 300 & 100 & 500 & 3 & 10 & 50 & 30 & 50 \\
\hline 140 & 3 & .7 & .07 & .5 & 300 & 100 & 700 & 3 & 10 & 70 & 20 & 70 \\
\hline 141 & 3 & 1 & .05 & .5 & 300 & 70 & 500 & 2 & L & 50 & 15 & 50 \\
\hline 154 & 3 & .7 & .07 & .3 & 300 & 30 & 700 & 2 & 7 & 50 & 20 & 50 \\
\hline 157 & 3 & .7 & .1 & .3 & 300 & 30 & 700 & 3 & 10 & 50 & 15 & 70 \\
\hline 159 & 3 & .7 & .07 & .7 & 300 & 50 & 700 & 2 & 10 & 50 & 20 & 70 \\
\hline 160 & 1.5 & .7 & .05 & .2 & 150 & 15 & 700 & 2 & 5 & 15 & $\mathrm{~L}$ & 20 \\
\hline 164 & 3 & 1 & .07 & .5 & 300 & 30 & 700 & 1.5 & 7 & 50 & 20 & 70 \\
\hline 172 & 3 & 1.5 & .05 & .5 & 300 & 20 & 700 & 3 & $\mathrm{~L}$ & 70 & 15 & 70 \\
\hline 179 & 3 & .7 & .07 & .7 & 300 & 50 & 700 & 2 & 7 & 50 & 15 & 30 \\
\hline 185 & 1.5 & 1 & .07 & .3 & 200 & 20 & 300 & 1.5 & 5 & 30 & 15 & 30 \\
\hline 208 & 3 & .7 & .05 & .5 & 200 & 30 & 700 & 2 & 10 & 70 & 15 & 70 \\
\hline 216 & 2 & .2 & .5 & .2 & 200 & L & 500 & 1.5 & 5 & 15 & 20 & 50 \\
\hline 218 & 2 & .7 & .3 & .3 & 500 & 15 & 500 & 1.5 & $\mathrm{~L}$ & 50 & 20 & 70 \\
\hline 220 & 3 & .7 & .1 & .3 & 300 & 70 & 500 & 1 & 7 & 50 & 20 & 70 \\
\hline 222 & 3 & .5 & .3 & 3 & 300 & 30 & 700 & 2 & 10 & 50 & 30 & 70 \\
\hline 228 & 3 & .5 & L & .3 & 150 & 10 & 500 & $i$ & L & 50 & 15 & 50 \\
\hline 229 & 2 & .5 & L & .2 & 20 & 30 & 700 & 1 & $\mathrm{~N}$ & 50 & 30 & 50 \\
\hline 230 & 3 & .7 & .05 & .3 & 300 & 50 & 700 & 1.5 & 7 & 50 & 30 & 70 \\
\hline 233 & 3 & .7 & .05 & .5 & 300 & 15 & 500 & 3 & $\mathrm{~N}$ & 70 & 20 & 30 \\
\hline 234 & 3 & .3 & .05 & .3 & 50 & 10 & 500 & 2 & $\mathrm{~N}$ & 50 & 15 & 50 \\
\hline 236 & 2 & .7 & .1 & .5 & 300 & 15 & 700 & 2 & L & 70 & 20 & 20 \\
\hline 215 & 3 & .7 & .15 & .3 & 300 & 15 & 700 & 1 & L & 50 & 30 & 50 \\
\hline 217 & 1.5 & .3 & .2 & .2 & 200 & 15 & 300 & 1.5 & 5 & 15 & 30 & 50 \\
\hline $219^{6}$ & 3 & .7 & .2 & .3 & 300 & 15 & 500 & 1.5 & 7 & 50 & 10 & 70 \\
\hline 226 & 1.5 & .2 & .3 & .2 & 100 & 10 & 200 & 1.5 & $i$ & 10 & 15 & 50 \\
\hline 227 & 1 & .2 & .2 & .15 & 150 & 10 & 300 & 1.5 & $\mathrm{~N}$ & 10 & 7 & 30 \\
\hline 232 & 3 & 1 & .07 & .3 & 300 & 30 & 500 & 3 & $L$ & 50 & 20 & N \\
\hline 248 & 3 & .5 & .2 & .5 & 300 & 20 & 500 & 1.5 & 5 & 50 & 7 & 70 \\
\hline 249 & 5 & 1 & .15 & .7 & 500 & 20 & 700 & 1.5 & 7 & 70 & 30 & 70 \\
\hline 251 & 3 & .7 & .15 & .3 & 300 & 20 & 700 & 1.5 & N & 50 & 20 & 70 \\
\hline 252 & 1 & .3 & .3 & .2 & 200 & 10 & 700 & $1 \cdot$ & 10 & 30 & 20 & 30 \\
\hline 255 & 3 & 1.5 & 2 & .7 & 700 & $\mathrm{~L}$ & 300 & 1 & 15 & 100 & 20 & 70 \\
\hline 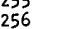 & 2 & $1^{\cdot 3}$ & .15 & .5 & 300 & 10 & 700 & 1 & L & 70 & 15 & 50 \\
\hline 257 & 2 & .5 & .3 & .2 & 200 & 20 & 700 & 1.5 & L & 70 & 10 & 50 \\
\hline 258 & 1.5 & .5 & $1^{\circ}$ & .3 & 200 & $\mathrm{~L}$ & 500 & 1.5 & $L$ & 30 & 10 & 50 \\
\hline 259 & 3 & .7 & .7 & .3 & 300 & 10 & 500 & 1.5 & L & 50 & 15 & 30 \\
\hline 260 & 2 & .5 & .7 & .3 & 200 & 30 & 500 & 1.5 & $\mathrm{~N}$ & 30 & 10 & 50 \\
\hline 261 & 3 & .7 & .05 & .5 & 300 & 30 & 500 & 1.5 & 5 & 70 & 30 & 50 \\
\hline 130 & 3 & .7 & .3 & .2 & 300 & 20 & 500 & 1.5 & 10 & 20 & 15 & $\begin{array}{l}50 \\
50\end{array}$ \\
\hline 014 & 3 & .7 & .05 & .3 & 200 & 30 & 500 & $\dot{L}$ & 15 & 30 & 30 & 50 \\
\hline 015 & 3 & .5 & .07 & .3 & 150 & L & 500 & 1 & 10 & 50 & L & 50 \\
\hline 019 & 1.5 & .3 & .07 & .2 & 200 & 15 & 300 & 1.5 & 7 & 30 & 10 & 50 \\
\hline 025 & 3 & 1.5 & .5 & .2 & 300 & 30 & 500 & 2 & 10 & 30 & 50 & $\begin{array}{l}30 \\
50\end{array}$ \\
\hline 028 & 2 & .7 & .05 & .2 & 150 & $L$ & 300 & L & $\mathrm{N}$ & 30 & 5 & 30 \\
\hline 029 & 1 & .3 & .07 & .15 & 100 & $L$ & 300 & $\mathrm{~L}$ & $\mathrm{~N}$ & 15 & 3 & L \\
\hline 036 & 1.5 & .5 & .1 & .15 & 200 & L & 300 & L & L & 20 & 10 & 30 \\
\hline 142 & 2 & .7 & .1 & .2 & 200 & 30 & 200 & 1 & 7 & 15 & 20 & 30 \\
\hline 214 & .7 & .5 & 1.5 & .2 & 100 & 15 & 70 & 2 & $\mathrm{~N}$ & 20 & 10 & 30 \\
\hline 225 & .5 & .2 & .15 & .1 & 100 & L & 500 & $\mathrm{~N}$ & $\mathrm{~N}$ & 5 & 7 & 20 \\
\hline 250 & .7 & .15 & .2 & .2 & 200 & 20 & 300 & 1 & $\underline{L}$ & 20 & 7 & 50 \\
\hline 262 & .7 & .2 & .1 & .2 & 100 & 15 & 500 & 1.5 & $\mathrm{~N}$ & 20 & 5 & 30 \\
\hline
\end{tabular}

See footnotes at end of table. 
of Belt rocks from the Pend Oreille area-Continued

\begin{tabular}{|c|c|c|c|c|c|c|c|c|c|c|}
\hline Sample & $\begin{array}{l}\mathrm{Nb} \\
(10)\end{array}$ & $\begin{array}{l}\mathrm{Ni} \\
(2)\end{array}$ & $\begin{array}{c}\mathrm{Pb} \\
(10)\end{array}$ & $\begin{array}{l}5 c \\
(5)\end{array}$ & $\begin{array}{c}\text { m) } \\
r \\
(50)\end{array}$ & $\begin{array}{c}v \\
(10)\end{array}$ & $\begin{array}{r}Y \\
(5) \\
\end{array}$ & $\begin{array}{c}2 r \\
(10)\end{array}$ & $\begin{array}{c}\text { Metamorphic } \\
\text { Grade }\end{array}$ & Sample description \\
\hline \multicolumn{11}{|c|}{ Prichard Formation--Cont inued } \\
\hline $\begin{array}{l}035 \\
037 \\
085 \\
132 \\
135\end{array}$ & $\begin{array}{r}L \\
10 \\
L \\
15 \\
L\end{array}$ & $\begin{array}{r}15 \\
7 \\
20 \\
30 \\
20\end{array}$ & $\begin{array}{r}10 \\
N \\
10 \\
30 \\
10\end{array}$ & $\begin{array}{r}15 \\
7 \\
7 \\
15 \\
10\end{array}$ & $\begin{array}{l}\mathrm{L} \\
N \\
N \\
N \\
L\end{array}$ & $\begin{array}{r}70 \\
50 \\
70 \\
70 \\
100\end{array}$ & $\begin{array}{l}30 \\
20 \\
30 \\
30 \\
30\end{array}$ & $\begin{array}{l}150 \\
150 \\
150 \\
150 \\
150\end{array}$ & $\begin{array}{l}\mathrm{Bb} \\
\mathrm{Bb} \\
\mathrm{Bb} \\
\mathrm{Bb} \\
\mathrm{Bb}\end{array}$ & $\begin{array}{c}\text { Argillite with andalusite. } \\
\text { Gray pyrrhotitic argillite. } \\
\text { Black and white argillite. } \\
\text { Do. } \\
\text { Slaty black argillite. }\end{array}$ \\
\hline $\begin{array}{l}136 \\
137 \\
138 \\
139 \\
140\end{array}$ & $\begin{array}{r}L \\
10 \\
10 \\
L \\
L\end{array}$ & $\begin{array}{r}15 \\
20 \\
L \\
30 \\
30\end{array}$ & $\begin{array}{r}L \\
10 \\
N \\
L \\
L\end{array}$ & $\begin{array}{r}15 \\
10 \\
7 \\
15 \\
15\end{array}$ & $\begin{array}{l}N \\
N \\
L \\
N \\
N\end{array}$ & $\begin{array}{r}100 \\
70 \\
70 \\
70 \\
70\end{array}$ & $\begin{array}{l}20 \\
30 \\
20 \\
30 \\
30\end{array}$ & $\begin{array}{l}150 \\
200 \\
150 \\
150 \\
150\end{array}$ & $\begin{array}{l}B b \\
B b \\
B b \\
B b \\
B b\end{array}$ & $\begin{array}{l}\text { Gray argillite. } \\
\text { Do. } \\
\text { Slaty black argillite. } \\
\text { Black and white argilite. } \\
\text { Do. }\end{array}$ \\
\hline $\begin{array}{l}141 \\
154 \\
157 \\
159 \\
160\end{array}$ & $\begin{array}{r}10 \\
10 \\
10 \\
10 \\
L\end{array}$ & $\begin{array}{r}\mathrm{L} \\
20 \\
30 \\
30 \\
15\end{array}$ & $\begin{array}{r}L \\
10 \\
L \\
20 \\
L\end{array}$ & $\begin{array}{r}10 \\
10 \\
15 \\
15 \\
7\end{array}$ & $\begin{array}{l}\mathrm{N} \\
\mathrm{N} \\
\mathrm{N} \\
\mathrm{N} \\
\mathrm{N}\end{array}$ & $\begin{array}{r}70 \\
70 \\
70 \\
100 \\
70\end{array}$ & $\begin{array}{l}30 \\
30 \\
50 \\
50 \\
20\end{array}$ & $\begin{array}{l}200 \\
150 \\
150 \\
150 \\
150\end{array}$ & $\begin{array}{l}B b \\
B b \\
B b \\
8 b \\
B b\end{array}$ & $\begin{array}{l}\text { Slaty black argillite. } \\
\text { Black and white argilitite. } \\
\text { Do. } \\
\text { Do. } \\
\text { Spotted black argillite. }\end{array}$ \\
\hline $\begin{array}{l}164 \\
172 \\
179 \\
185 \\
208\end{array}$ & $\begin{array}{l}L \\
10 \\
15 \\
10 \\
15\end{array}$ & $\begin{array}{r}30 \\
5 \\
20 \\
20 \\
20\end{array}$ & $\begin{array}{r}L \\
L \\
L \\
50 \\
N\end{array}$ & $\begin{array}{l}15 \\
15 \\
10 \\
10 \\
15\end{array}$ & $\begin{array}{l}N \\
N \\
N \\
N \\
N\end{array}$ & $\begin{array}{r}70 \\
100 \\
70 \\
70 \\
70\end{array}$ & $\begin{array}{l}30 \\
30 \\
30 \\
30 \\
30\end{array}$ & $\begin{array}{l}150 \\
100 \\
150 \\
150 \\
150\end{array}$ & $\begin{array}{l}\mathrm{Bb} \\
\mathrm{Bb} \\
\mathrm{Bb} \\
\mathrm{Bb} \\
\mathrm{Bb}\end{array}$ & $\begin{array}{l}\text { Black and white argillite. } \\
\text { Spotted black argillite. } \\
\text { Black and white argillite. } \\
\text { Spotted black argillite. } \\
\text { Black and white argillite. }\end{array}$ \\
\hline $\begin{array}{l}216 \\
218 \\
220 \\
222 \\
228\end{array}$ & $\begin{array}{c}10 \\
10 \\
10 \\
10 \\
L\end{array}$ & $\begin{array}{r}10 \\
L \\
15 \\
30 \\
7\end{array}$ & $\begin{array}{r}\mathrm{L} \\
10 \\
\mathrm{~L} \\
10 \\
\mathrm{~L}\end{array}$ & $\begin{array}{r}7 \\
10 \\
15 \\
15 \\
10\end{array}$ & $\begin{array}{l}\mathrm{L} \\
\mathrm{L} \\
\mathrm{N} \\
\mathrm{N} \\
\mathrm{N}\end{array}$ & $\begin{array}{l}50 \\
70 \\
70 \\
70 \\
70\end{array}$ & $\begin{array}{l}30 \\
30 \\
30 \\
30 \\
30\end{array}$ & $\begin{array}{l}150 \\
150 \\
150 \\
150 \\
100\end{array}$ & $\begin{array}{l}\mathrm{Bb} \\
\mathrm{Bb} \\
\mathrm{Bb} \\
\mathrm{Bb} \\
\mathrm{Bb}\end{array}$ & $\begin{array}{l}\text { Do. } \\
\text { Do. } \\
\text { Do. } \\
\text { Do. } \\
\text { Do. }\end{array}$ \\
\hline
\end{tabular}

Gray argillite.

Black and white argillite. Do.

Do.

Schistose argillite.

Gneissic argillite.

Schistose argillite.

Gneissic argillite.

Do.

Do.

Black and white gneiss.

Black and white schist.

Coarse mica schist.

Coarse mica gneiss.

Biotite gneiss.

Black and white schist.

Gneissic argillite.

Gneissic silty argillite.

Schistose argillite.

Gneissic argillite.

Schistose argillite.

Gray argillitic siltite.

Gray siltite.

Dark gray siltite.

Gray argillitic siltite.

Gray pyrrhotitic siltite.

Spotted siltite.

Do.

Salt and pepper siltite.

$\begin{array}{rrrrrrrrrl}142 & L & 20 & 15 & 5 & N & 50 & 20 & 100 & B b \\ 214 & 10 & L & L & 5 & 200 & 50 & 30 & 150 & B g n\end{array}$

8 lack and white siltite.

Gneissic siltite.

Do.

Do.

Do. 
$\mathrm{T}_{\mathrm{ABLE}}$ 3.-Semiquantitative spectrographic analyses

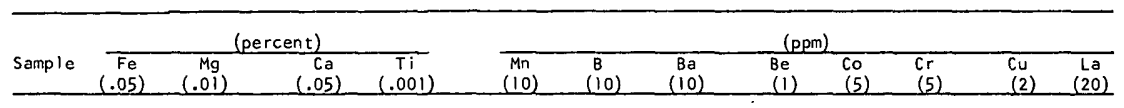

Prichard Formation--Continued

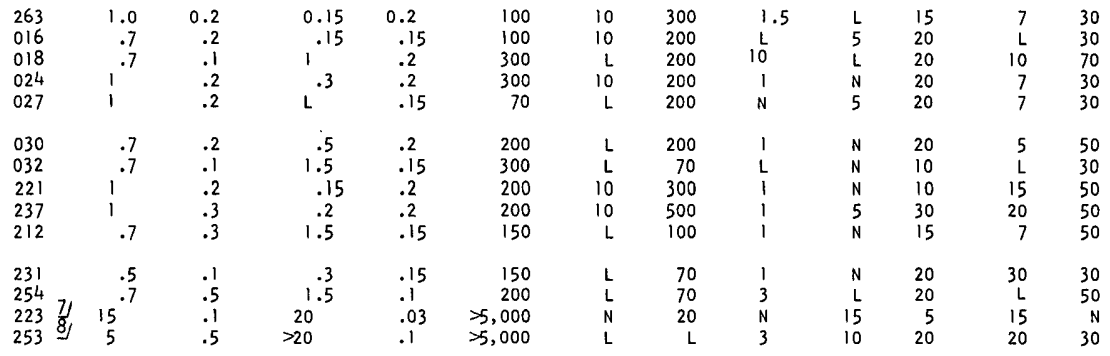

1) Reported as $L(5)$ for Mo.

2) Reported as L(200) for $\mathrm{Zn}$.

3) Contains $0.7 \mathrm{ppm} \mathrm{Ag}$.

4) Contains $20 \mathrm{ppm} \mathrm{Mo}, 0.7 \mathrm{ppm} \mathrm{Ag}$, and $10 \mathrm{ppm} \mathrm{Bi}$. 
of Belt rocks from the Pend Oreille area-Continued

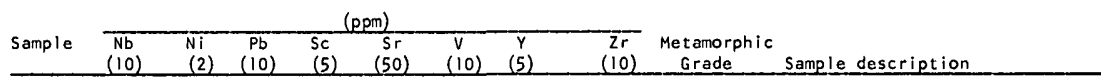

Prichard Formation--Cont inued

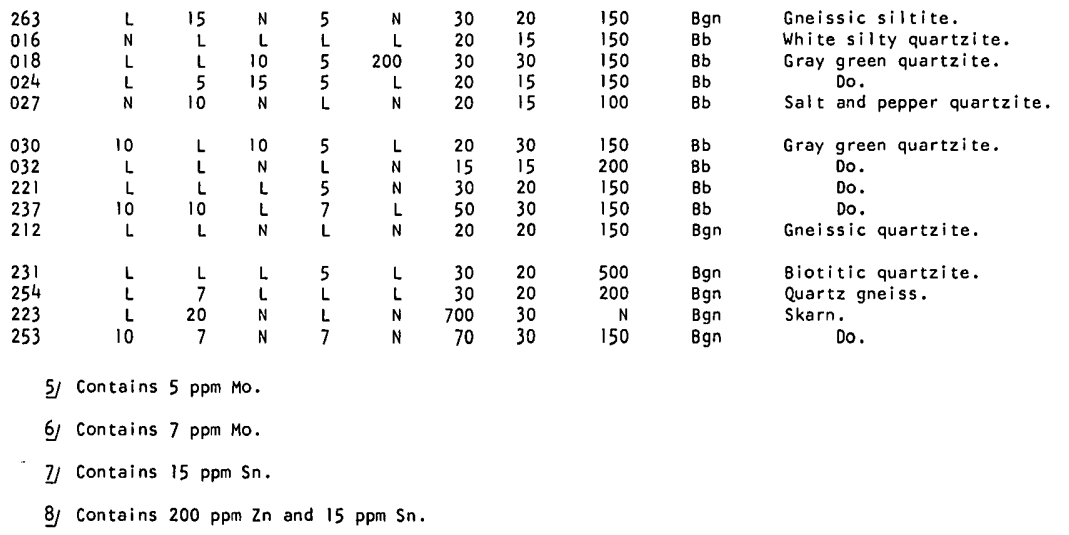


TABLE 4.-Replicate semiquantitative spectrographic

[Number in parentheses indicates sensitivity limit of method used. L, undetermined amount of for but not found in any sample were As(200), Au(10), Cd(20), Sb(100), $\mathrm{Sn}(10), \mathrm{W}(50)$,
$\mathrm{N}(10)$ for Bi, and $\mathrm{N}(5)$ for Mo. D. J. Grimes, analyst]

\begin{tabular}{|c|c|c|c|c|c|c|c|c|c|}
\hline \multirow[b]{2}{*}{ Sample } & \multicolumn{9}{|c|}{ (percent) } \\
\hline & $\begin{array}{l}\mathrm{Fe} \\
(.05) \\
\end{array}$ & $\begin{array}{l}\mathrm{Mg} \\
(.01) \\
\end{array}$ & $\begin{array}{c}\mathrm{Ca} \\
(.05) \\
\end{array}$ & $\begin{array}{l}\mathrm{Ti} \\
(.001)\end{array}$ & $\begin{array}{c}M n \\
(10) \\
\end{array}$ & $\begin{array}{c}\mathrm{B} \\
(10) \\
\end{array}$ & $\begin{array}{c}\mathrm{Ba} \\
(10)\end{array}$ & $\begin{array}{l}8 e \\
\text { (1) }\end{array}$ & $\begin{array}{l}\text { Cc } \\
\text { (5) } \\
\end{array}$ \\
\hline $\begin{array}{l}001 \\
011\end{array}$ & $2^{1.5}$ & $\begin{array}{l}0.7 \\
1.5\end{array}$ & $\begin{array}{r}0.05 \\
.05\end{array}$ & $\begin{array}{c}0.15 \\
.3\end{array}$ & $\begin{array}{l}50 \\
70\end{array}$ & $\begin{array}{l}30 \\
70\end{array}$ & $\begin{array}{l}300 \\
500\end{array}$ & $\begin{array}{l}1.0 \\
1.5\end{array}$ & $\begin{array}{l}10 \\
15\end{array}$ \\
\hline $\begin{array}{l}012 \\
022\end{array}$ & $\begin{array}{l}3 \\
3\end{array}$ & $\begin{array}{l}1.5 \\
1.5\end{array}$ & $\begin{array}{c}\mathrm{L} \\
.05\end{array}$ & $\begin{array}{l}.2 \\
.3\end{array}$ & $\begin{array}{r}70 \\
100\end{array}$ & $\begin{array}{l}50 \\
50\end{array}$ & $\begin{array}{l}300 \\
500\end{array}$ & 1.5 & $\begin{array}{l}10 \\
10\end{array}$ \\
\hline $\begin{array}{l}023 \\
033\end{array}$ & $3^{1.5}$ & 1.5 & $\begin{array}{l}.2 \\
.5\end{array}$ & $\begin{array}{l}.2 \\
.15\end{array}$ & $\begin{array}{l}200 \\
300\end{array}$ & $\begin{array}{l}10 \\
15\end{array}$ & $\begin{array}{l}500 \\
500\end{array}$ & L & $\begin{array}{r}7 \\
10\end{array}$ \\
\hline $\begin{array}{l}034 \\
044\end{array}$ & $\begin{array}{l}3 \\
3\end{array}$ & 1.5 & $\begin{array}{l}.7 \\
.5\end{array}$ & $\begin{array}{l}.3 \\
.3\end{array}$ & $\begin{array}{l}500 \\
300\end{array}$ & $\begin{array}{l}15 \\
15\end{array}$ & $\begin{array}{l}500 \\
300\end{array}$ & 1.5 & $\begin{array}{l}5 \\
5\end{array}$ \\
\hline $\begin{array}{l}045 \\
055\end{array}$ & $\begin{array}{l}1.5 \\
1.5\end{array}$ & .1 & $\begin{array}{l}.07 \\
.05\end{array}$ & $\begin{array}{l}.2 \\
.2\end{array}$ & $\begin{array}{l}70 \\
50\end{array}$ & $\begin{array}{l}15 \\
15\end{array}$ & $\begin{array}{l}200 \\
200\end{array}$ & $\begin{array}{l}1 \\
\mathrm{~L}\end{array}$ & $\begin{array}{l}5 \\
5\end{array}$ \\
\hline $\begin{array}{l}056 \\
066\end{array}$ & $\begin{array}{l}2 \\
2\end{array}$ & $\begin{array}{l}1.5 \\
1.5\end{array}$ & .1 & $\begin{array}{l}.2 \\
.3\end{array}$ & $\begin{array}{l}30 \\
20\end{array}$ & $\begin{array}{l}30 \\
20\end{array}$ & $\begin{array}{l}300 \\
300\end{array}$ & $\mathrm{~L}$ & L \\
\hline $\begin{array}{l}067 \\
077\end{array}$ & $\begin{array}{l}3 \\
3\end{array}$ & $2^{1.5}$ & $\begin{array}{l}.07 \\
.07\end{array}$ & $\begin{array}{l}.3 \\
.3\end{array}$ & $\begin{array}{l}15 \\
20\end{array}$ & $\begin{array}{l}150 \\
300\end{array}$ & $\begin{array}{l}700 \\
700\end{array}$ & $2^{1.5}$ & $\begin{array}{l}L \\
5\end{array}$ \\
\hline $\begin{array}{l}078 \\
088\end{array}$ & $2^{1.5}$ & $\begin{array}{l}3 \\
2\end{array}$ & $\begin{array}{l}.1 \\
.1\end{array}$ & $\begin{array}{l}.2 \\
.2\end{array}$ & $\begin{array}{l}50 \\
50\end{array}$ & $\begin{array}{l}70 \\
70\end{array}$ & $\begin{array}{l}300 \\
500\end{array}$ & $\begin{array}{l}1.5 \\
1.5\end{array}$ & $\begin{array}{l}7 \\
7\end{array}$ \\
\hline $\begin{array}{l}089 \\
099\end{array}$ & $\begin{array}{l}3 \\
2\end{array}$ & $\begin{array}{l}.7 \\
.7\end{array}$ & $\begin{array}{l}.07 \\
.07\end{array}$ & $\begin{array}{l}.2 \\
.2\end{array}$ & $\begin{array}{l}500 \\
300\end{array}$ & $\begin{array}{l}20 \\
20\end{array}$ & $\begin{array}{l}300 \\
500\end{array}$ & 1 & $\begin{array}{l}10 \\
10\end{array}$ \\
\hline $\begin{array}{l}091 \\
101\end{array}$ & ${ }^{1} .7$ & $\begin{array}{l}.3 \\
.2\end{array}$ & $L^{.05}$ & $\begin{array}{l}.2 \\
.15\end{array}$ & $\begin{array}{l}200 \\
150\end{array}$ & $\begin{array}{l}15 \\
10\end{array}$ & $\begin{array}{l}300 \\
200\end{array}$ & 1.5 & $\underline{L}$ \\
\hline $\begin{array}{l}102 \\
112\end{array}$ & $\begin{array}{l}3 \\
5\end{array}$ & $\begin{array}{l}1.5 \\
1.5\end{array}$ & $\begin{array}{l}.07 \\
.07\end{array}$ & $\begin{array}{l}1 \\
1\end{array}$ & $\begin{array}{l}15 \\
15\end{array}$ & $\begin{array}{l}300 \\
500\end{array}$ & $\begin{array}{l}700 \\
700\end{array}$ & $\begin{array}{l}2 \\
3\end{array}$ & $\begin{array}{l}5 \\
5\end{array}$ \\
\hline $\begin{array}{l}113 \\
123\end{array}$ & $\begin{array}{l}3 \\
5\end{array}$ & $3^{1.5}$ & $\begin{array}{l}.05 \\
.15\end{array}$ & $\begin{array}{l}.3 \\
.7\end{array}$ & $\begin{array}{l}15 \\
50\end{array}$ & $\begin{array}{l}100 \\
200\end{array}$ & $\begin{array}{l}500 \\
700\end{array}$ & $2^{1.5}$ & $\begin{array}{l}5 \\
7\end{array}$ \\
\hline $\begin{array}{l}124 \frac{1}{2} \\
134\end{array}$ & $\begin{array}{l}2 \\
1.5\end{array}$ & $\begin{array}{r}10 \\
7\end{array}$ & $\begin{array}{l}20 \\
20\end{array}$ & $\begin{array}{l}.15 \\
.15\end{array}$ & $\begin{array}{l}5,000 \\
3,000\end{array}$ & $\begin{array}{l}500 \\
700\end{array}$ & $\begin{array}{l}500 \\
300\end{array}$ & $\mathrm{~L}$ & $\begin{array}{l}\mathrm{L} \\
\mathrm{N}\end{array}$ \\
\hline $\begin{array}{l}135 \\
145\end{array}$ & $\begin{array}{l}3 \\
3\end{array}$ & $\begin{array}{r}.7 \\
1.5\end{array}$ & $\begin{array}{l}.3 \\
.5\end{array}$ & $\begin{array}{l}.5 \\
.5\end{array}$ & $\begin{array}{l}500 \\
500\end{array}$ & $\begin{array}{l}30 \\
50\end{array}$ & $\begin{array}{l}700 \\
700\end{array}$ & $\begin{array}{l}3 \\
2\end{array}$ & $\begin{array}{l}10 \\
10\end{array}$ \\
\hline $\begin{array}{l}146 \\
156\end{array}$ & $\begin{array}{l}.5 \\
.5\end{array}$ & $\begin{array}{l}.5 \\
.3\end{array}$ & $\begin{array}{l}10 \\
15\end{array}$ & $\begin{array}{l}.15 \\
.15\end{array}$ & $\begin{array}{l}300 \\
300\end{array}$ & $\begin{array}{l}15 \\
10\end{array}$ & $\begin{array}{l}500 \\
500\end{array}$ & $\begin{array}{l}\mathrm{L} \\
\mathrm{L}\end{array}$ & $\begin{array}{l}\mathrm{L} \\
\mathrm{L}\end{array}$ \\
\hline $\begin{array}{l}157 \\
167\end{array}$ & $\begin{array}{l}3 \\
3\end{array}$ & $1^{.7}$ & .1 & $\begin{array}{r}.3 \\
.3\end{array}$ & $\begin{array}{l}300 \\
300\end{array}$ & $\begin{array}{l}30 \\
30\end{array}$ & $\begin{array}{l}700 \\
700\end{array}$ & $\begin{array}{l}3 \\
2\end{array}$ & $\begin{array}{l}10 \\
10\end{array}$ \\
\hline $\begin{array}{l}168 \\
178\end{array}$ & $\begin{array}{l}3 \\
3\end{array}$ & $\begin{array}{l}5 \\
3\end{array}$ & $\begin{array}{l}.1 \\
.07\end{array}$ & $\begin{array}{l}.3 \\
.5\end{array}$ & $\begin{array}{l}50 \\
50\end{array}$ & $\begin{array}{l}70 \\
50\end{array}$ & $\begin{array}{l}500 \\
500\end{array}$ & $\begin{array}{l}2 \\
2\end{array}$ & $\begin{array}{r}10 \\
7\end{array}$ \\
\hline $\begin{array}{l}179 \\
189\end{array}$ & $\begin{array}{l}3 \\
3\end{array}$ & $\begin{array}{l}.7 \\
.7\end{array}$ & $\begin{array}{l}.07 \\
.07\end{array}$ & $\begin{array}{l}.7 \\
.3\end{array}$ & $\begin{array}{l}300 \\
300\end{array}$ & $\begin{array}{l}50 \\
30\end{array}$ & $\begin{array}{l}700 \\
700\end{array}$ & $\begin{array}{l}2 \\
1.5\end{array}$ & $\begin{array}{l}7 \\
5\end{array}$ \\
\hline $\begin{array}{l}181 \\
191\end{array}$ & $\begin{array}{l}.7 \\
.7\end{array}$ & $\begin{array}{l}.3 \\
.3\end{array}$ & ${ }^{\mathrm{L}} .05$ & $\begin{array}{l}.3 \\
.2\end{array}$ & $\begin{array}{l}20 \\
15\end{array}$ & $\begin{array}{l}\mathrm{L} \\
\mathrm{L}\end{array}$ & $\begin{array}{l}150 \\
150\end{array}$ & $\begin{array}{l}1 \\
\text { L }\end{array}$ & $\begin{array}{l}\mathrm{N} \\
\mathrm{L}\end{array}$ \\
\hline $\begin{array}{l}192 \\
202\end{array}$ & $\begin{array}{l}5 \\
5\end{array}$ & $\begin{array}{l}1 \\
1\end{array}$ & $\begin{array}{l}.07 \\
.07\end{array}$ & $\begin{array}{l}.2 \\
.2\end{array}$ & $\begin{array}{l}300 \\
300\end{array}$ & $\begin{array}{l}30 \\
30\end{array}$ & $\begin{array}{l}150 \\
150\end{array}$ & 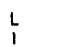 & $\begin{array}{l}10 \\
10\end{array}$ \\
\hline $\begin{array}{l}203 \\
213\end{array}$ & $\begin{array}{l}.3 \\
.3\end{array}$ & $\begin{array}{l}.1 \\
.07\end{array}$ & $\mathrm{~L}$ & $\begin{array}{l}.05 \\
.05\end{array}$ & $\begin{array}{l}15 \\
70\end{array}$ & $\begin{array}{r}\mathrm{L} \\
10\end{array}$ & $\begin{array}{l}200 \\
150\end{array}$ & $L$ & ${ }_{N}^{N}$ \\
\hline $\begin{array}{l}214 \\
224\end{array}$ & $\begin{array}{l}.7 \\
.5\end{array}$ & $\begin{array}{l}.5 \\
.3\end{array}$ & $\begin{array}{r}1.5 \\
.7\end{array}$ & $\begin{array}{l}.2 \\
.15\end{array}$ & $\begin{array}{l}100 \\
100\end{array}$ & $\begin{array}{r}15 \\
\mathrm{~L}\end{array}$ & $\begin{array}{l}70 \\
50\end{array}$ & $\begin{array}{l}2 \\
1.5\end{array}$ & $\begin{array}{l}\mathrm{N} \\
\mathrm{N}\end{array}$ \\
\hline $\begin{array}{l}225 \\
235\end{array}$ & $\begin{array}{l}.5 \\
.7\end{array}$ & $\begin{array}{l}.2 \\
.3\end{array}$ & $\begin{array}{l}.15 \\
.2\end{array}$ & $\begin{array}{r}.1 \\
.2\end{array}$ & $\begin{array}{l}100 \\
200\end{array}$ & 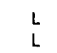 & $\begin{array}{l}500 \\
500\end{array}$ & $\begin{array}{l}\mathrm{N} \\
\mathrm{L}\end{array}$ & $\begin{array}{l}N \\
N\end{array}$ \\
\hline
\end{tabular}

1) Reported as $0.7 \mathrm{ppm} \mathrm{Ag}, 10 \mathrm{ppm} \mathrm{Bi}$, and $20 \mathrm{ppm} \mathrm{Mo.}$ 
analyses of Belt rocks from the Pend Oreille area

element present below sensitivity limit; $N$, element was looked for but not found. Also looked and $\mathrm{Zn}(200)$. Except as indicated by footnotes, all samples were reported as $\mathbf{N}(0.5)$ for $\mathrm{Ag}$,

\begin{tabular}{|c|c|c|c|c|c|c|c|c|c|c|c|}
\hline \multirow[b]{2}{*}{ Sample } & \multicolumn{11}{|c|}{ (ppm) } \\
\hline & $\begin{array}{l}r \\
(5) \\
\end{array}$ & $\begin{array}{l}\mathrm{Cu} \\
\text { (2) }\end{array}$ & $\begin{array}{l}\mathrm{La} \\
(20) \\
\end{array}$ & $\begin{array}{c}\mathrm{Nb} \\
(10)\end{array}$ & $\begin{array}{l}\mathrm{Ni} \\
(2)\end{array}$ & $\begin{array}{l}\mathrm{Pb} \\
(10)\end{array}$ & $\begin{array}{l}\mathrm{Sc} \\
\text { (5) }\end{array}$ & $\begin{array}{c}5 r \\
(50)\end{array}$ & $\begin{array}{c}v \\
(10)\end{array}$ & $\begin{array}{c}y \\
(5)\end{array}$ & $\begin{array}{r}\mathrm{zr} \\
(10) \\
\end{array}$ \\
\hline $\begin{array}{l}001 \\
011\end{array}$ & $\begin{array}{l}30 \\
30\end{array}$ & $\begin{array}{l}5 \\
7\end{array}$ & $\begin{array}{l}50 \\
70\end{array}$ & $\begin{array}{l}\mathrm{N} \\
\mathrm{N}\end{array}$ & $\begin{array}{l}30 \\
30\end{array}$ & $\begin{array}{l}L \\
L\end{array}$ & $\begin{array}{c}7 \\
10\end{array}$ & $\begin{array}{l}\mathrm{N} \\
\mathrm{N}\end{array}$ & $\begin{array}{l}50 \\
70\end{array}$ & $\begin{array}{l}30 \\
30\end{array}$ & $\begin{array}{l}150 \\
150\end{array}$ \\
\hline $\begin{array}{l}012 \\
022\end{array}$ & $\begin{array}{l}50 \\
70\end{array}$ & $\begin{array}{l}30 \\
50\end{array}$ & $\begin{array}{l}50 \\
70\end{array}$ & $\begin{array}{l}10 \\
15\end{array}$ & $\begin{array}{l}20 \\
30\end{array}$ & $\begin{array}{l}N \\
L\end{array}$ & $\begin{array}{l}10 \\
10\end{array}$ & $\begin{array}{l}\mathrm{N} \\
\mathrm{N}\end{array}$ & $\begin{array}{l}50 \\
70\end{array}$ & $\begin{array}{l}20 \\
30\end{array}$ & $\begin{array}{l}100 \\
150\end{array}$ \\
\hline $\begin{array}{l}023 \\
033\end{array}$ & $\begin{array}{l}30 \\
30\end{array}$ & $\begin{array}{l}10 \\
15\end{array}$ & $\begin{array}{l}50 \\
30\end{array}$ & $L$ & $\begin{array}{l}20 \\
30\end{array}$ & $\begin{array}{l}15 \\
15\end{array}$ & $\begin{array}{l}7 \\
7\end{array}$ & $\begin{array}{l}\mathrm{N} \\
\mathrm{N}\end{array}$ & $\begin{array}{l}50 \\
50\end{array}$ & $\begin{array}{l}15 \\
20\end{array}$ & $\begin{array}{r}70 \\
150\end{array}$ \\
\hline $\begin{array}{l}034 \\
044\end{array}$ & $\begin{array}{l}70 \\
50\end{array}$ & $\begin{array}{l}7 \\
5\end{array}$ & $\begin{array}{l}70 \\
70\end{array}$ & $\begin{array}{r}\mathrm{L} \\
10\end{array}$ & $\begin{array}{r}7 \\
10\end{array}$ & $\begin{array}{l}15 \\
15\end{array}$ & $\begin{array}{l}10 \\
10\end{array}$ & $\begin{array}{r}200 \\
150\end{array}$ & $\begin{array}{l}70 \\
70\end{array}$ & $\begin{array}{l}30 \\
30\end{array}$ & $\begin{array}{l}150 \\
100\end{array}$ \\
\hline $\begin{array}{l}045 \\
055\end{array}$ & $\begin{array}{l}20 \\
15\end{array}$ & $\begin{array}{l}5 \\
5\end{array}$ & $\begin{array}{l}30 \\
30\end{array}$ & $\begin{array}{l}L \\
L\end{array}$ & $\begin{array}{l}5 \\
5\end{array}$ & ${ }_{N}^{N}$ & $\begin{array}{l}5 \\
5\end{array}$ & $\begin{array}{l}\mathrm{N} \\
\mathrm{N}\end{array}$ & $\begin{array}{l}30 \\
30\end{array}$ & $\begin{array}{l}20 \\
20\end{array}$ & $\begin{array}{l}150 \\
150\end{array}$ \\
\hline $\begin{array}{l}056 \\
066\end{array}$ & $\begin{array}{l}30 \\
20\end{array}$ & $\begin{array}{l}7 \\
5\end{array}$ & $\begin{array}{l}20 \\
20\end{array}$ & $\underline{L}$ & $\begin{array}{l}30 \\
20\end{array}$ & $\begin{array}{l}\mathrm{N} \\
\mathrm{N}\end{array}$ & $\begin{array}{l}7 \\
7\end{array}$ & $\begin{array}{l}\mathrm{N} \\
\mathrm{N}\end{array}$ & $\begin{array}{l}50 \\
50\end{array}$ & $\begin{array}{l}20 \\
20\end{array}$ & $\begin{array}{l}150 \\
150\end{array}$ \\
\hline $\begin{array}{l}067 \\
077\end{array}$ & $\begin{array}{l}50 \\
70\end{array}$ & $\mathbf{L}$ & $\begin{array}{l}50 \\
50\end{array}$ & 10 & $\begin{array}{l}10 \\
15\end{array}$ & L & $\begin{array}{l}10 \\
15\end{array}$ & $\begin{array}{l}\mathrm{N} \\
\mathrm{N}\end{array}$ & $\begin{array}{l}70 \\
70\end{array}$ & $\begin{array}{l}30 \\
30\end{array}$ & $\begin{array}{l}200 \\
200\end{array}$ \\
\hline $\begin{array}{l}078 \\
088\end{array}$ & $\begin{array}{l}30 \\
30\end{array}$ & 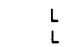 & $\begin{array}{l}30 \\
50\end{array}$ & $\begin{array}{r}10 \\
L\end{array}$ & $\begin{array}{l}20 \\
20\end{array}$ & $\mathrm{~N}_{\mathrm{N}}^{\mathrm{N}}$ & $\begin{array}{l}7 \\
5\end{array}$ & $\begin{array}{l}\mathrm{N} \\
\mathrm{N}\end{array}$ & $\begin{array}{l}50 \\
50\end{array}$ & $\begin{array}{l}30 \\
30\end{array}$ & $\begin{array}{l}150 \\
150\end{array}$ \\
\hline $\begin{array}{l}089 \\
099\end{array}$ & $\begin{array}{l}30 \\
30\end{array}$ & $\begin{array}{l}10 \\
15\end{array}$ & $\begin{array}{l}30 \\
30\end{array}$ & $\mathrm{~L}_{\mathrm{L}}^{\mathrm{N}}$ & $\begin{array}{l}20 \\
30\end{array}$ & $\begin{array}{l}10 \\
15\end{array}$ & $\begin{array}{l}5 \\
5\end{array}$ & $\begin{array}{l}\mathrm{N} \\
\mathrm{N}\end{array}$ & $\begin{array}{l}30 \\
50\end{array}$ & $\begin{array}{l}15 \\
20\end{array}$ & $\begin{array}{l}150 \\
150\end{array}$ \\
\hline $\begin{array}{l}091 \\
101\end{array}$ & $\begin{array}{l}15 \\
15\end{array}$ & $\begin{array}{l}7 \\
\mathrm{~L}\end{array}$ & $\begin{array}{l}30 \\
20\end{array}$ & $\mathrm{~L}$ & $\begin{array}{l}5 \\
5\end{array}$ & $\begin{array}{l}\mathrm{N} \\
\mathrm{N}\end{array}$ & $\begin{array}{l}5 \\
5\end{array}$ & $\begin{array}{l}\mathrm{N} \\
\mathrm{N}\end{array}$ & $\begin{array}{l}20 \\
30\end{array}$ & $\begin{array}{l}20 \\
15\end{array}$ & $\begin{array}{l}150 \\
100\end{array}$ \\
\hline $\begin{array}{l}102 \\
112\end{array}$ & $\begin{array}{l}50 \\
50\end{array}$ & $\begin{array}{l}\mathrm{L} \\
\mathrm{L}\end{array}$ & $\begin{array}{l}50 \\
70\end{array}$ & $\begin{array}{r}\mathrm{L} \\
10\end{array}$ & $\begin{array}{r}7 \\
30\end{array}$ & $\stackrel{N}{\mathrm{~N}}$ & $\begin{array}{l}15 \\
15\end{array}$ & $\begin{array}{l}\mathrm{N} \\
\mathrm{N}\end{array}$ & $\begin{array}{l}70 \\
70\end{array}$ & $\begin{array}{l}50 \\
50\end{array}$ & $\begin{array}{l}200 \\
200\end{array}$ \\
\hline $\begin{array}{l}113 \\
123\end{array}$ & $\begin{array}{r}70 \\
100\end{array}$ & $\begin{array}{l}7 \\
7\end{array}$ & $\begin{array}{l}50 \\
70\end{array}$ & $\begin{array}{l}10 \\
10\end{array}$ & $\begin{array}{l}15 \\
20\end{array}$ & L & $\begin{array}{r}7 \\
15\end{array}$ & $\begin{array}{l}\mathrm{N} \\
\mathrm{N}\end{array}$ & $\begin{array}{r}70 \\
100\end{array}$ & $\begin{array}{l}30 \\
70\end{array}$ & $\begin{array}{l}200 \\
300\end{array}$ \\
\hline $\begin{array}{l}124 \\
134\end{array}$ & $\begin{array}{l}30 \\
20\end{array}$ & $\begin{array}{l}3,000 \\
3,000\end{array}$ & $\begin{array}{l}30 \\
30\end{array}$ & $L$ & $\begin{array}{l}15 \\
15\end{array}$ & $\stackrel{N}{L}_{\mathrm{L}}$ & $\begin{array}{l}5 \\
L\end{array}$ & $\begin{array}{l}\mathrm{N} \\
\mathrm{N}\end{array}$ & $\begin{array}{l}50 \\
50\end{array}$ & $\begin{array}{l}30 \\
30\end{array}$ & $\begin{array}{l}150 \\
100\end{array}$ \\
\hline $\begin{array}{l}135 \\
145\end{array}$ & $\begin{array}{l}50 \\
50\end{array}$ & $\begin{array}{l}10 \\
10\end{array}$ & $\begin{array}{l}50 \\
70\end{array}$ & $\begin{array}{r}\mathrm{L} \\
10\end{array}$ & $\begin{array}{l}20 \\
30\end{array}$ & $\begin{array}{l}10 \\
10\end{array}$ & $\begin{array}{l}10 \\
10\end{array}$ & $L$ & $\begin{array}{r}100 \\
70\end{array}$ & $\begin{array}{l}30 \\
30\end{array}$ & $\begin{array}{l}150 \\
150\end{array}$ \\
\hline $\begin{array}{l}146 \\
156\end{array}$ & $\begin{array}{l}15 \\
15\end{array}$ & $\begin{array}{l}7 \\
5\end{array}$ & $\begin{array}{l}30 \\
50\end{array}$ & L & $L$ & $\begin{array}{r}10 \\
\mathrm{~L}\end{array}$ & $\frac{L}{5}$ & $\begin{array}{r}100 \\
\mathrm{~L}\end{array}$ & $\begin{array}{l}20 \\
20\end{array}$ & $\begin{array}{l}30 \\
30\end{array}$ & $\begin{array}{l}100 \\
100\end{array}$ \\
\hline $\begin{array}{l}157 \\
167\end{array}$ & $\begin{array}{l}50 \\
50\end{array}$ & $\begin{array}{l}15 \\
20\end{array}$ & $\begin{array}{l}70 \\
70\end{array}$ & $\begin{array}{l}10 \\
10\end{array}$ & $\begin{array}{l}30 \\
30\end{array}$ & $\frac{L}{10}$ & $\begin{array}{l}15 \\
10\end{array}$ & $\begin{array}{l}\mathrm{N} \\
\mathrm{N}\end{array}$ & $\begin{array}{r}70 \\
100\end{array}$ & $\begin{array}{l}50 \\
50\end{array}$ & $\begin{array}{l}150 \\
150\end{array}$ \\
\hline $\begin{array}{l}168 \\
178\end{array}$ & $\begin{array}{l}30 \\
50\end{array}$ & $\frac{L}{N}$ & $\begin{array}{l}50 \\
70\end{array}$ & $\frac{1}{10}$ & $\begin{array}{l}20 \\
20\end{array}$ & $\begin{array}{l}\mathrm{N} \\
\mathrm{N}\end{array}$ & $\begin{array}{l}10 \\
10\end{array}$ & $\begin{array}{l}\mathrm{N} \\
\mathrm{N}\end{array}$ & $\begin{array}{l}70 \\
70\end{array}$ & $\begin{array}{l}30 \\
30\end{array}$ & $\begin{array}{l}150 \\
150\end{array}$ \\
\hline $\begin{array}{l}179 \\
189\end{array}$ & $\begin{array}{l}50 \\
30\end{array}$ & $\begin{array}{l}15 \\
10\end{array}$ & $\begin{array}{l}30 \\
20\end{array}$ & $\begin{array}{l}15 \\
10\end{array}$ & $\begin{array}{l}20 \\
20\end{array}$ & L & $\begin{array}{l}10 \\
10\end{array}$ & $\begin{array}{l}\mathrm{N} \\
\mathrm{N}\end{array}$ & $\begin{array}{l}70 \\
70\end{array}$ & $\begin{array}{l}30 \\
30\end{array}$ & $\begin{array}{l}150 \\
150\end{array}$ \\
\hline $\begin{array}{l}181 \\
191\end{array}$ & $\begin{array}{l}7 \\
7\end{array}$ & $\begin{array}{r}N \\
15\end{array}$ & $\begin{array}{l}50 \\
50\end{array}$ & $\begin{array}{r}10 \\
L\end{array}$ & $L$ & $\mathrm{~N}_{\mathrm{N}}^{\mathrm{N}}$ & $\begin{array}{l}7 \\
5\end{array}$ & $\begin{array}{l}\mathrm{N} \\
\mathrm{N}\end{array}$ & $\begin{array}{l}30 \\
20\end{array}$ & $\begin{array}{l}20 \\
20\end{array}$ & $\begin{array}{r}1,000 \\
700\end{array}$ \\
\hline $\begin{array}{l}192 \\
202\end{array}$ & $\begin{array}{l}20 \\
30\end{array}$ & $\begin{array}{l}30 \\
30\end{array}$ & $\begin{array}{l}30 \\
50\end{array}$ & $\begin{array}{l}10 \\
10\end{array}$ & $\begin{array}{l}30 \\
30\end{array}$ & L & $\begin{array}{c}7 \\
10\end{array}$ & $\begin{array}{l}N \\
N\end{array}$ & $\begin{array}{l}50 \\
70\end{array}$ & $\begin{array}{l}30 \\
30\end{array}$ & $\begin{array}{l}150 \\
200\end{array}$ \\
\hline $\begin{array}{l}203 \\
213\end{array}$ & $\begin{array}{l}7 \\
5\end{array}$ & $\begin{array}{l}\mathrm{L} \\
7\end{array}$ & $\begin{array}{l}\mathrm{L} \\
30\end{array}$ & $\stackrel{N}{L}$ & $\mathrm{~L}$ & $\stackrel{N}{N}_{N}^{N}$ & $\stackrel{L}{N}$ & $\begin{array}{l}\mathrm{N} \\
\mathrm{N}\end{array}$ & $\begin{array}{r}15 \\
15\end{array}$ & $\begin{array}{l}15 \\
15\end{array}$ & $\begin{array}{l}100 \\
100\end{array}$ \\
\hline $\begin{array}{l}214 \\
224\end{array}$ & $\begin{array}{r}20 \\
7\end{array}$ & $\begin{array}{r}10 \\
7\end{array}$ & $\begin{array}{l}30 \\
30\end{array}$ & $\begin{array}{r}10 \\
\mathrm{~L}\end{array}$ & $\stackrel{L}{L}$ & $L$ & $\begin{array}{l}5 \\
5\end{array}$ & $\begin{array}{l}200 \\
100\end{array}$ & $\begin{array}{l}50 \\
20\end{array}$ & $\begin{array}{l}30 \\
20\end{array}$ & $\begin{array}{l}150 \\
150\end{array}$ \\
\hline $\begin{array}{l}225 \\
235\end{array}$ & $\begin{array}{r}5 \\
20\end{array}$ & $\begin{array}{r}7 \\
15\end{array}$ & $\begin{array}{l}20 \\
20\end{array}$ & $L$ & $\mathrm{~L}$ & $\begin{array}{l}20 \\
15\end{array}$ & L & $\begin{array}{l}\mathrm{N} \\
\mathrm{N}\end{array}$ & $\begin{array}{l}15 \\
50\end{array}$ & $\begin{array}{l}15 \\
20\end{array}$ & $\begin{array}{l}150 \\
150\end{array}$ \\
\hline
\end{tabular}

2) Reported as $0.7 \mathrm{ppm} \mathrm{Ag,} 10 \mathrm{ppm} \mathrm{Bi}$, and $15 \mathrm{ppm} \mathrm{Mo.}$ 
Precision of the sampling and analytical technique was measured by analysis of blind replicates of every eleventh sample from the Pend Oreille area. The replicate samples, which include a range of element concentrations, were taken from a 1-pint crushed control sample representing a split of the 2-pound or larger sample collected of a given rock type. The precision thus includes errors in sampling as well as errors inherent in the preparation and analysis of each sample. Analytical results on the 23 pairs of replications are given in table 4.

Analytical precision is shown graphically in figure 4. The solid bars show the number of replicate samples having identical analyses for a given element; the open bars show the number of replicate samples differing by one or more steps in the reported results. Step differences were determined by comparing values from the first split with those from the second. Values reported as $\mathbf{N}$ (no line seen for the elements) and $\mathrm{L}$ (element present in an amount less than the sensitivity limit) were arbitrarily counted as two and one steps below the limit of sensitivity, respectively, for purposes of comparison. However, a pair of N's was excluded as a valid measure of similarity. For elements having a relatively high limit of sensitivity (many reported N's or L's), these arbitrary comparisons will tend to result in an artificially high precision. Such elements as $\mathrm{Pb}$ and $\mathrm{Sr}$ are particularly suspect in this regard. The precision of $\mathrm{Nb}$ and $\mathrm{Co}$ and perhaps of $\mathrm{Be}, \mathrm{Ni}$, and $\mathrm{Cu}$ should also be viewed with caution.

The remaining elements exhibit quite satisfactory precisions in that more than 80 percent of the comparisons fall within plus or minus one step. Of particular interest, however, is the occasional large difference reported for replicates of $\mathrm{Mn}, \mathrm{Cr}, \mathrm{Cu}$, and $\mathrm{Ni}$. To the extent that such disparity is not due strictly to analytical inprecision, it may be due to the particulate character of small amounts of minerals containing those elements in the sample. Such errors then, may reflect sample inhomogeneity which, in turn, may provide clues for interpretation of element distributions in these rocks.

One factor aids in maintaining high precision for the spectrographic method. A split of sample G-1 was shot on each plate as a reference standard. If the $\mathrm{G}-1$ values were not sufficiently close to the known values, then that plate was discarded and another made. Values of G-1 for 12 plates used for the Pend Oreille samples are given in table 5. If we assume that the bottle of G-1 used as the reference standard in this study was an exact duplicate of the original $\mathrm{G}-1$, then table 5 can be used by other workers to estimate accuracy of the reported values. The table also shows that our selected use of $\mathrm{N}$ and $\mathrm{L}$ in the calculations for table 4 is reasonable, considering the "best value" content of elements in G-1 that are below the sensitivity limit of the method we used. In other words, $L$ on table 5 is reported for elements whose true value is about one reporting step below the sensitivity limit, and $\mathrm{N}$ is reported for elements more than one step 


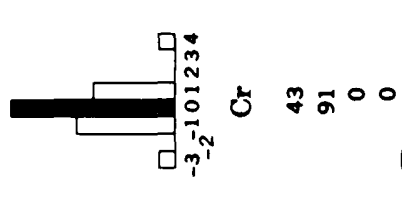

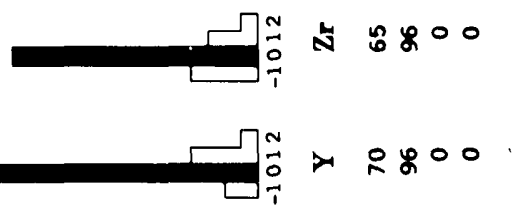

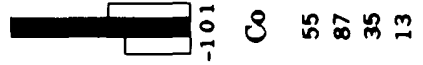

$\square$

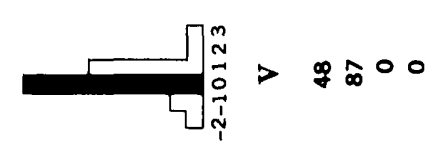

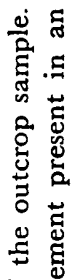

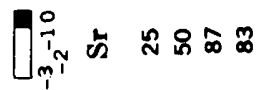

잉

ثิ

$\square=2$ ก

—

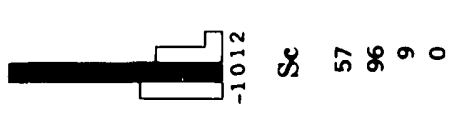

के म्न

ฐี ్ㅠㅇ

ד

อิ के

:

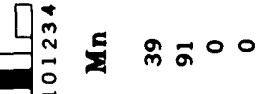

居
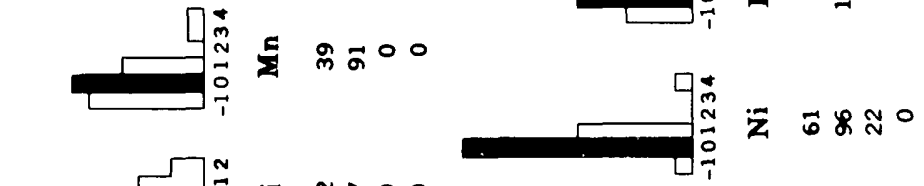

正

잉

Z

岁

L

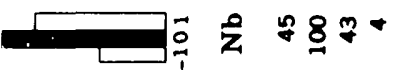

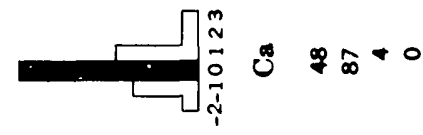
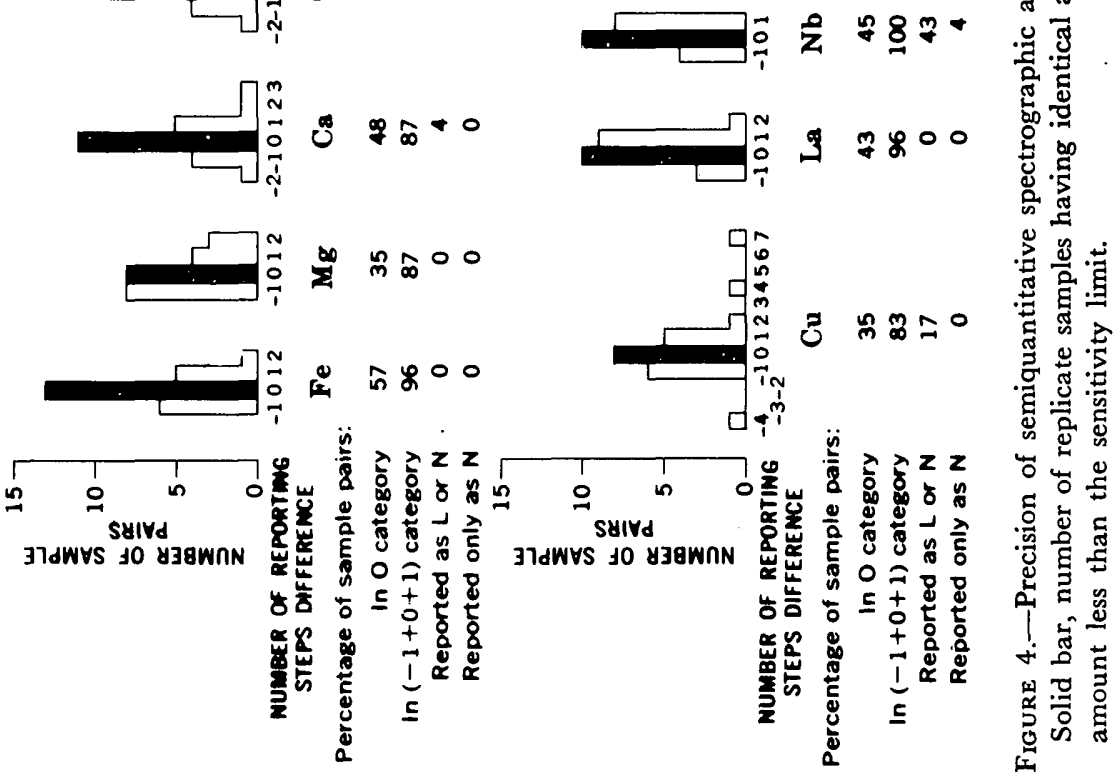
below. Thus L's and N's for the same sample are probably only about one reporting step apart, but two N's could reflect any value from about two steps below the sensitivity limit down to zero.

Finally, high-calcium samples alter the excitation conditions of the arc in the spectrographic method used here. Comparison with mineralogic and wet chemical data indicates that values reported as 10 percent and more calcium are consistently low. This suggests that in high-calcium samples trace elements may also be affected by the altered arcing conditions, which could result in a slight positive or negative bias for a given element. This then limits the usefulness of the analytical method on carbonate rocks; the precision of the method is sufficiently good to permit comparison of elements among groups of carbonate rocks, but the potentially poor accuracy may not permit comparison with low-calcium rocks. As a consequence, our geologic analysis is restricted to the pelitic and psammitic rocks of low carbonate content.

Calculations of average contents and dispersions are based on logarithms because the analytical data are reported as midpoints of geometric classes (Miesch, 1967, p. B4). In this study, the average content is estimated by the geometric mean (the antilog of the mean logarithm), and the dispersion or scatter is estimated by the geometric deviation (the antilog of the standard deviation of the $\log$ values). Geometric means and geometric deviations for elements in various groups of Belt rocks are given for the Mission Mountains Primitive Area in table 6 and for the Pend Oreille area in table 7. For purposes of these computations, real, but arbitrary, numbers were substituted for the nonnumeric codes $\mathrm{N}, \mathrm{L}$, and $>$ (greater than) used in reporting the raw data. (See table 3.) Table 8 lists the numbers substituted and the percentage of the substitution needed for each element. The particular numbers used for $\mathrm{L}$ and $>$ were determined largely on the basis of wet chemical or quantitative spectrographic analyses of some samples. Numbers substituted for $\mathrm{N}$ are simply small positive numbers between the limit of sensitivity and zero. Use of logarithms precludes the use of zero. Following common practice, results of calculations are given to two significant figures.

\section{MINERALOGY OF ROCK TYPES}

Mineral data on most of the clastic rocks of the Mission Mountains (table 2) and Pend Oreille (Harrison and Campbell, 1963, table 1) areas are summarized in figure 5 . Rock groups containing less than 3 samples are not shown. The ratios among quartz, potassium feldspar, and plagioclase for the siltites shown in figure 5, as well as for the carbonatic siltites from the Pend Oreille area, are summarized in figure 6.

The general uniformity of the Belt rocks is apparent in figure 5; most rocks are graywacke. The grain size and mineralogy are directly related. 

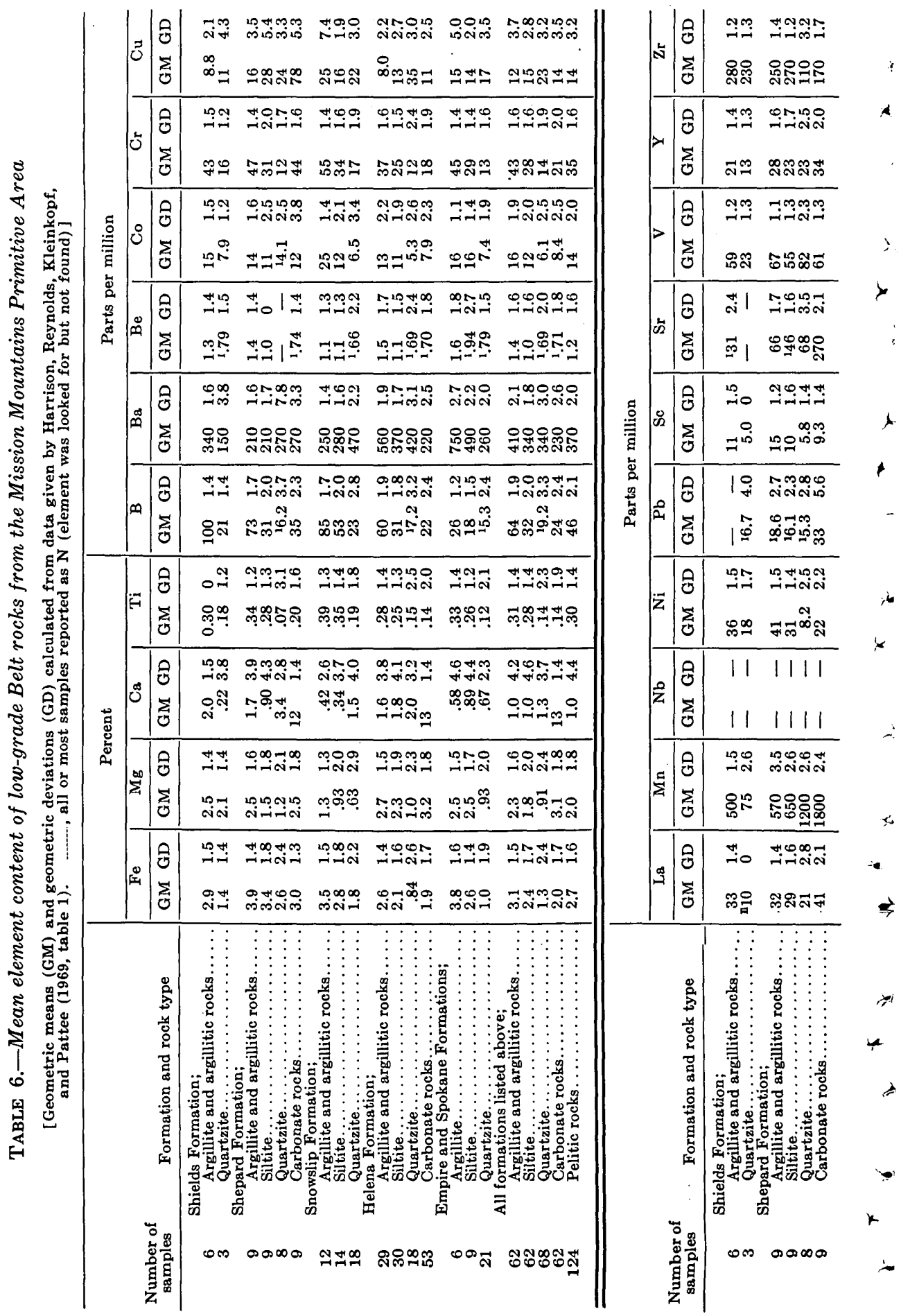


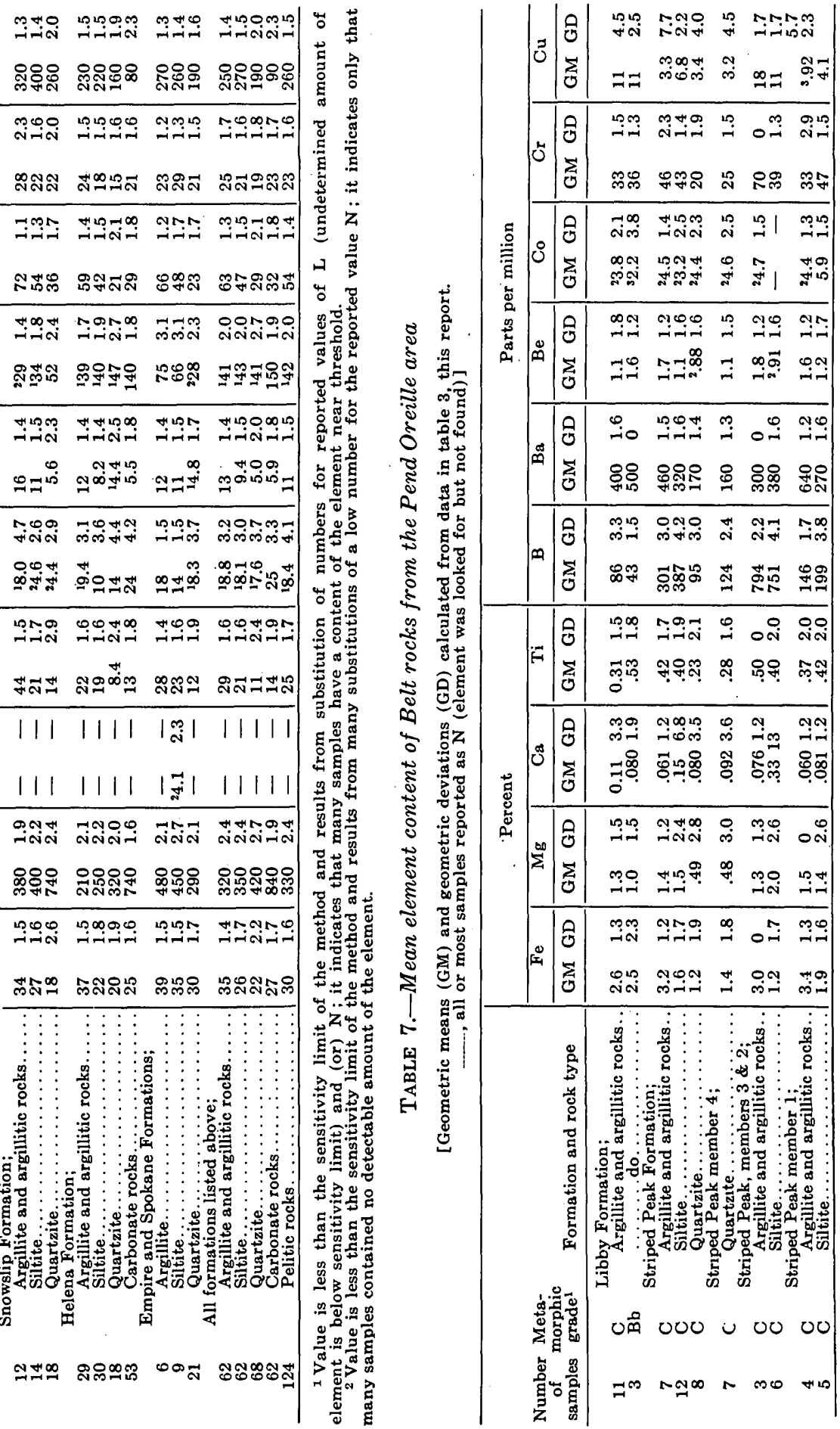




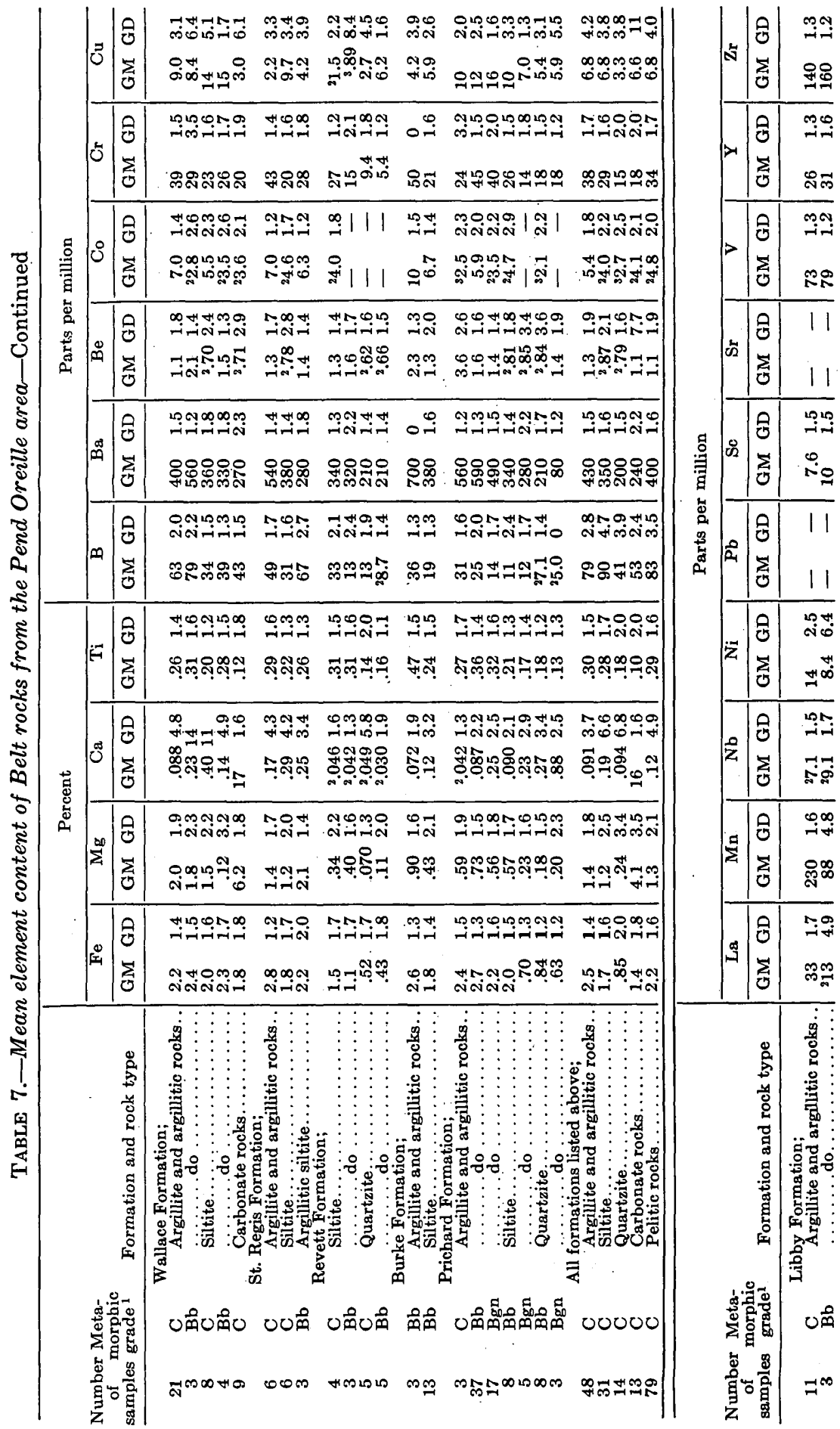




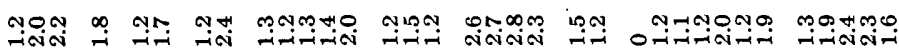

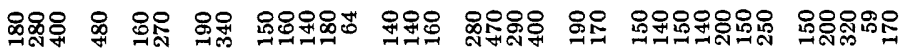

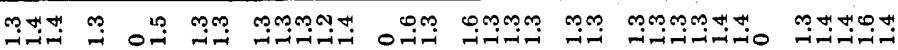

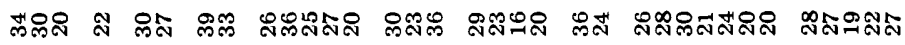

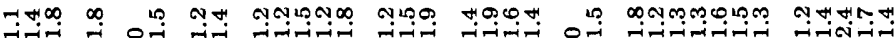

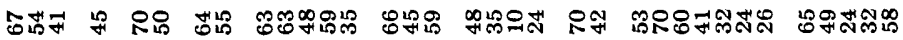

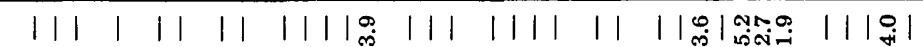

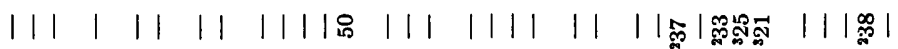

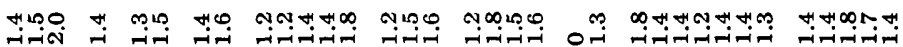
아 \# म

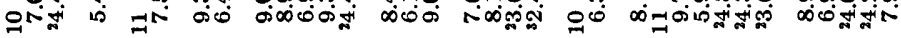

\begin{tabular}{|c|c|c|c|c|c|c|c|c|c|}
\hline$|\underset{\infty}{\infty}|$ & 1 & $\log _{\sim}^{\infty}$ & 11 & 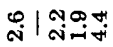 & 111 & 1111 & $\left.\right|_{\text {di }} ^{*}$ & 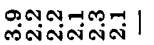 & 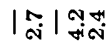 \\
\hline$|\stackrel{0}{\infty}|$ & 1 & |욤 & 11 & | & 111 & 1111 & I & 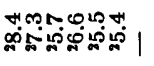 & $\mid \overrightarrow{|r|}$ \\
\hline ن & $\stackrel{0}{\infty}$ & 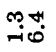 & : & 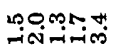 & 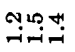 & 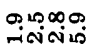 & mo. & 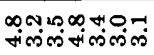 & 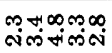 \\
\hline فำ & 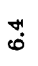 & @्ల & $\stackrel{\infty}{\infty}$ & ลี유 ${ }^{-\infty}{ }^{\infty}$ & 유퓨 & تُ & $\ddot{N}$ & 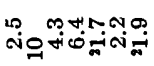 & 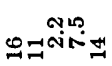 \\
\hline 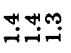 & $\stackrel{m}{-1}$ & تُ & 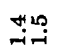 & | & تهم & 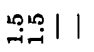 & $\underset{\text { ma }}{m}$ & 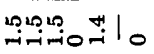 & نه \\
\hline فำ & นด & 유: & 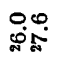 & 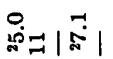 & $\dddot{\infty}_{0}^{\infty} \overbrace{0}^{\infty}$ & 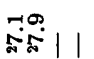 & $=\stackrel{n}{ }$ & 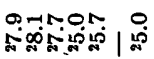 & |ृ \\
\hline 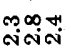 & $\stackrel{0}{\sim}$ & ผึ & Oृ & 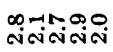 & 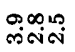 & & 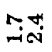 & 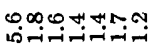 & 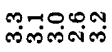 \\
\hline 눙ㅇ & 8 & ถ่ำ & ज्ञ & 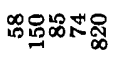 & 응으므 & ఉંన్లిథ & ৪\% & 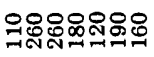 & 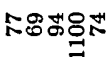 \\
\hline קิ & $\stackrel{4}{-!}$ & ヘุฒ & 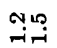 & 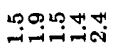 & গำ & 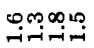 & ه & & \\
\hline 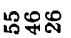 & బొ & 유요 & చే'థ & ำำ & వ্నిణ & 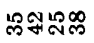 & 유과 & 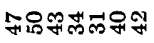 & 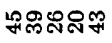 \\
\hline
\end{tabular}

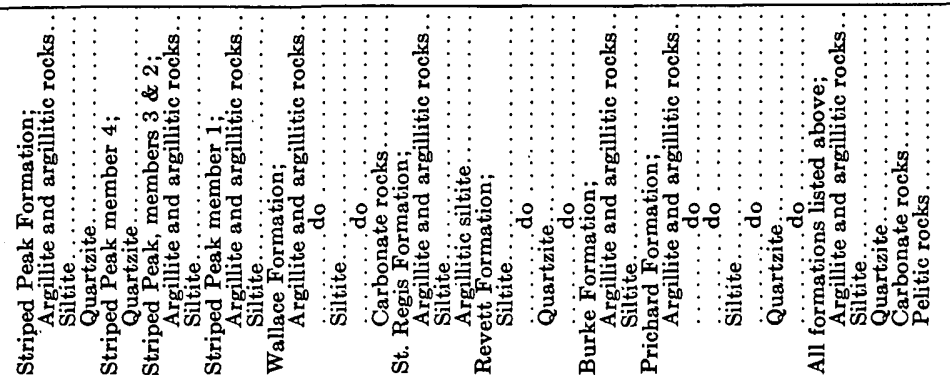

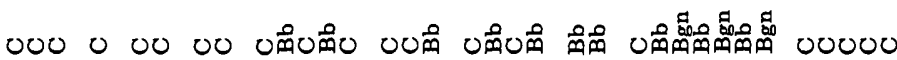

N్ㅐ

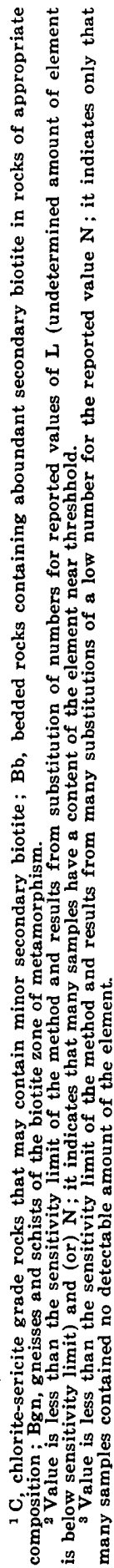


TABLE 8. -Numbers used to replace nonnumeric codes $(N, L$, and $>$ ) prior to geochemical calculations

[N, element looked for but not found: $L$, undetermined amount of element present below sensitivity limit; and $>$, undetermined amount of element present above the number shown. $\mathrm{Mg}_{\mathrm{g}}$ and $\mathrm{Ca}$, in percent; other elements, in parts per million]

\begin{tabular}{|c|c|c|c|c|c|c|}
\hline \multirow[b]{2}{*}{ Element } & \multicolumn{2}{|c|}{$\mathrm{N}$} & \multicolumn{2}{|c|}{ L } & \multicolumn{2}{|c|}{$>$} \\
\hline & $\begin{array}{c}\text { Replacement } \\
\text { number }\end{array}$ & $\begin{array}{l}\text { Percentage of } \\
\text { samples } \\
\text { reported } \\
\text { as code }\end{array}$ & $\begin{array}{c}\text { Replacement } \\
\text { number }\end{array}$ & $\begin{array}{l}\text { Percentage of } \\
\text { samples } \\
\text { reported } \\
\text { as code }\end{array}$ & $\begin{array}{c}\text { Replacement } \\
\text { number }\end{array}$ & $\begin{array}{l}\text { Percentage of } \\
\text { samples } \\
\text { reported } \\
\text { as code }\end{array}$ \\
\hline $\mathrm{Mg}$ & - & - & - & - & 15 & 0.4 \\
\hline $\mathrm{Ca}$ & 0.01 & 0.6 & 0.03 & 5.4 & 30 & 1.2 \\
\hline Mn & . & & & - & 7,000 & 1.0 \\
\hline B & 1 & 2.1 & 5 & 8.5 & & - \\
\hline $\mathrm{Ba}$ & - & & 5 & .2 & 10,000 & .4 \\
\hline $\mathrm{Be}$ & .1 & 2.9 & .5 & 20.7 & - & - \\
\hline Co & 1 & 12.0 & 3 & 12.0 & - & - \\
\hline $\mathrm{Cr}$ & 1 & .2 & 3 & .2 & - & - \\
\hline $\mathrm{Cu}$ & .1 & .4 & 1 & 10.2 & - & - \\
\hline $\mathrm{La}$ & 3 & 2.7 & 10 & 4.8 & - & - \\
\hline $\mathrm{Nb}$ & 1 & 44.4 & 5 & 30.3 & - & - \\
\hline $\mathrm{Ni}$ & .1 & .6 & 1 & 10.8 & - & - \\
\hline $\mathrm{Pb}$ & 2 & 30.9 & 5 & 26.8 & - & - \\
\hline Sc & 1 & 2.9 & 3 & .6 & - & - \\
\hline $\mathrm{Sr}$ & 10 & 45.6 & 30 & 17.2 & - & - \\
\hline $\mathrm{V}$ & - & - & 5 & 1.0 & - & - \\
\hline $\mathbf{Y}$ & - & - & - & - & 300 & .2 \\
\hline$\overline{\mathrm{Z}} \mathrm{r}$ & 1 & .2 & - & - & 1,500 & .2 \\
\hline
\end{tabular}

The mudstones from the Mission Mountains, except for those from the Empire and Spokane Formations, probably were deposited nearer an old shoreline and are somewhat siltier than those of the Pend Oreille area. Siltites and quartzites of the Pend Oreille area tend to be slightly more feldspathic than their stratigraphic equivalents in the Mission Mountains. The striking contrast between the Belt mudstones and the Pierre Shale, which many Belt rocks resemble in outcrop and hand specimen, is brought out by figure 5 . The position of the Pierre Shale in figure 5 was calculated from information given in table 5 of a report on this shale by Tourtelot (1962). The Pierre also differs from the Belt in that montmorillonite is a principal clay mineral (montmorillonite was included with illite for the calculations used to arrive at the Pierre Shale position in figure 5). Ratios among quartz, potassium feldspar, and plagioclase for Belt siltites are remarkably uniform (fig. 6), even for rocks containing several percent of carbonate minerals, which form the cement.

Mineralogic comparisons that include carbonate minerals in clastic Belt rocks from the two study areas are shown in figure 7 . The data used here are from table 2 of this report for the Mission Mountains rocks, from table 1 of Harrison and Campbell (1963) for some Pend Oreille rocks-plus additional data on low-grade metamorphic rocks in the Pend Oreille area accumulated since 1963 - and from table 5 of Tourtelot (1962). Here again, 


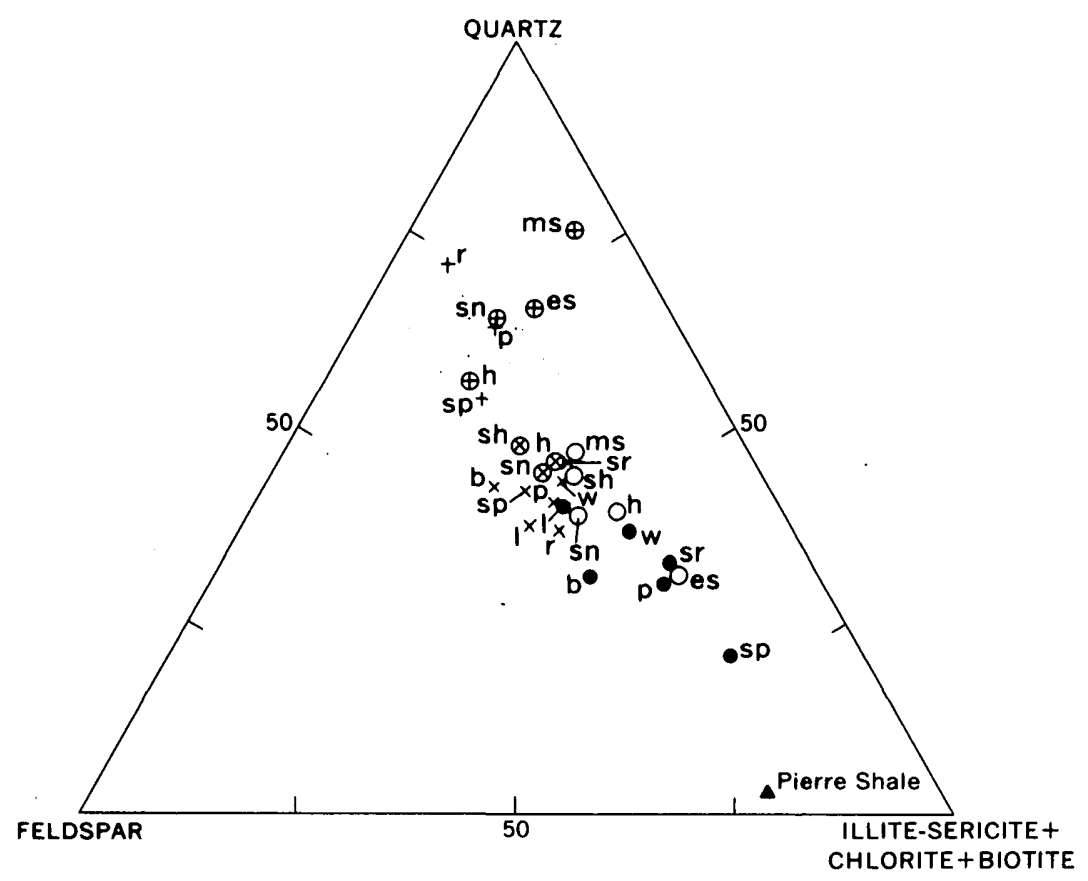

\begin{tabular}{|c|c|c|c|}
\hline \multirow[t]{2}{*}{ Formation } & \multicolumn{3}{|c|}{ Number of samples } \\
\hline & $\begin{array}{l}\text { rgillite } \\
\text { ale (O) }\end{array}$ & Siltite $(X)$ & Quartzite (+) \\
\hline \multicolumn{4}{|c|}{ Pend Oreille area } \\
\hline Libby (1) & 4 & 4 & $\ldots$. \\
\hline Striped Peak (sp). & 3 & 11 & 9 \\
\hline Wallace (w) & 12 & 16 & $\ldots$. \\
\hline 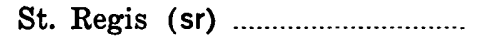 & 6 & 4 & $\cdots$ \\
\hline Revett (r) & $\ldots$ & 5 & 10 \\
\hline Burke (b) & 3 & 14 & $\ldots .$. \\
\hline Prichard (p) & 11 & 7 & 6 \\
\hline \multicolumn{4}{|c|}{ Mission Mountains Primitive Area } \\
\hline Shields (ms) & 3 & $\ldots$. & 3 \\
\hline Shepard (sh) & 5 & 3 & $\cdots$. \\
\hline Snowslip (sn) & 6 & 5 & 5 \\
\hline 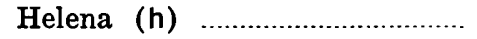 & 5 & 13 & 6 \\
\hline Empire and Spokane (es) ........... & 3 & $\cdots$ & 7 \\
\hline
\end{tabular}

Figure 5.-Average modes, in volume percent, of rocks of the Belt Supergroup, Pend Oreille area (symbol not circled), and Mission Mountains Primitive Area (symbol circled). 


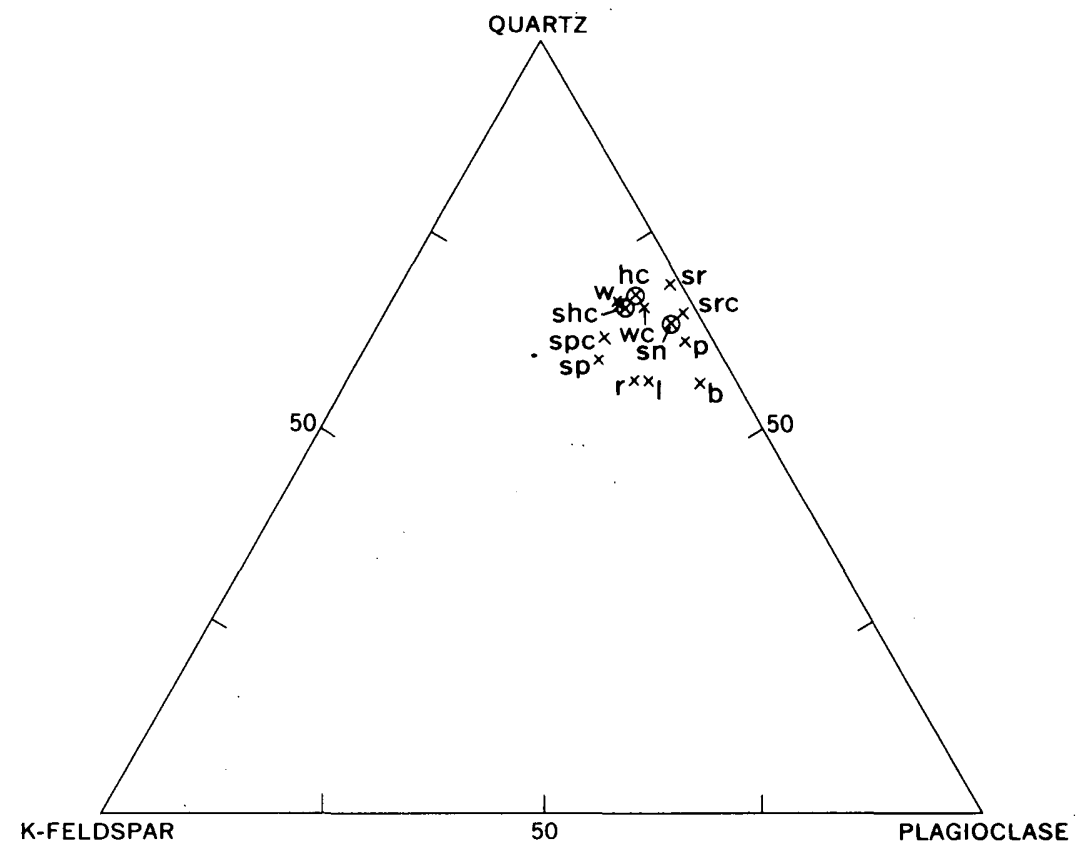

\begin{tabular}{ll}
\hline Formation & Number of samples \\
\hline & Pend Oreille area
\end{tabular}

Libby (1) 4

Striped Peak (sp) 11

Striped Peak; dolomitic (spc)

Wallace $(w)$

Wallace; dolomitic and calcareous (wc)

St. Regis (sr)

St. Regis; dolomitic (src)

Revett ( $r$ )

Burke (b)

Prichard ( $p)$

Shepard; dolomitic and calcareous (shc)

Snowslip (sn)

Helena; calcareous and dolomitic (hc)

FIGURE 6.-Ratio among quartz, potassium feldspar, and plagioclase (volume percent) in siltite of the Belt Supergroup, Pend Oreille area (symbol not circled) and Mission Mountains Primitive Area (symbol circled). c, siltite contains 10 percent or more of carbonate minerals. 


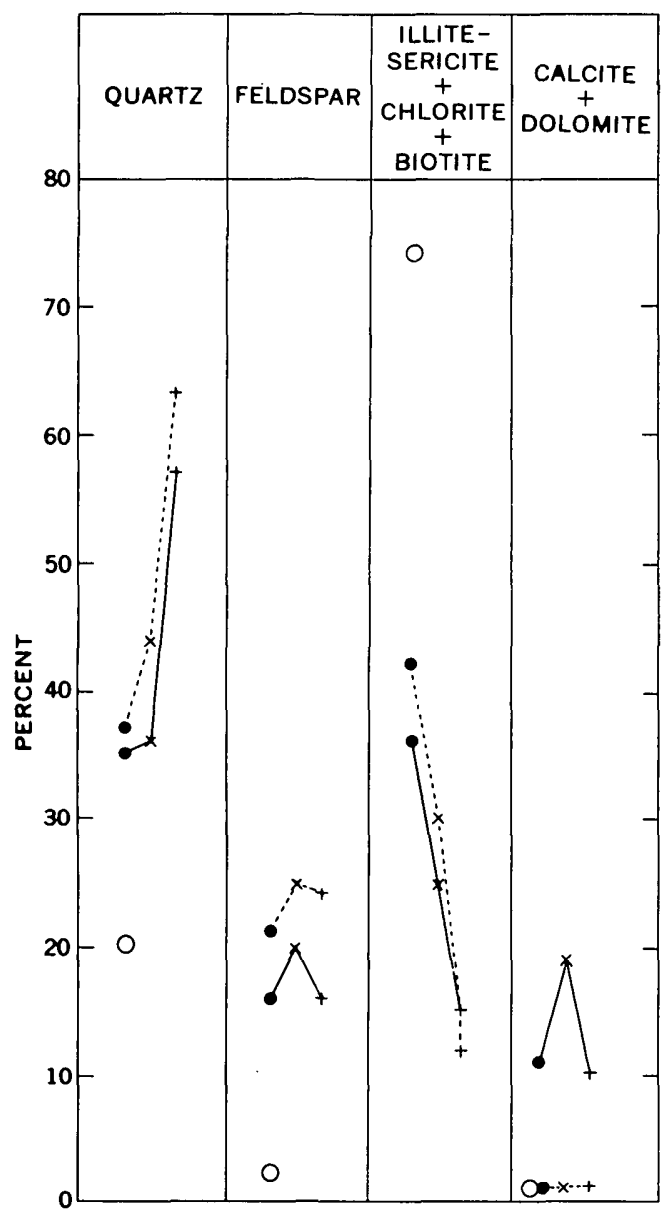

EXPLANATION

Belt argillite plus laminated argillite and siltite

Belt siltite

$+$

Belt quartzite

0

Pierre Shale

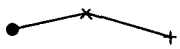

Mission Mountain rocks (Empire and Spokane through Shields Formations)

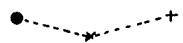

Pend Oreille area rocks (Prichard through Libby Formations)

Figure 7.-Average mineralogy of clastic Belt rocks. Number of samples from Mission Mountains: argillite, 24; siltite, 25; quartzite, 23. Number of samples from Pend Oreille area: argillite, 120 ; siltite, 51 ; quartzite, 20. Also shown for comparison: Pierre Shale, 20 samples.

only slight differences of the Belt rocks from the two widely separated areas are evident. A greater carbonate minerals content (also noticeable in the field) in the Mission Mountains strata is the most striking difference, although the feldspar content of the Pend Oreille rocks is slightly greater than that of the Mission Mountain rocks. Sharp contrast between Belt "shales" and the Pierre Shale is again apparent. The pronounced decrease in the clay mineral-mica-chlorite component with increasing rock grain size is a significant factor in the distribution of many minor elements.

\section{GEOCHEMISTRY}

The geometric deviations for most elements in a rock type represented 
by five or more samples with real (not coded) values is about the same. In contrast, the geometric deviation of most elements tends to increase with increasing rock grain size. Thus the quartzites tend to be less homogeneous chemically than the siltites, which in turn tend to be less homogeneous than the argillites. The increased chemical variability of the coarser grained rocks could reflect small-scale variation (differences between closely spaced samples) or large-scale variation (differences from place to place) or both. Field evidence supports the conclusion that the coarser rocks are actually more variable in composition from place to place.

A comparison of the average element content of the four Belt rock types and the metamorphically equivalent (lowest grade) Belt rocks from the two study areas is shown in figure 8. The averages (geometric means) are taken from tables 6 and 7 . The clastic rocks from each area are plotted in order of increasing grain size from left to right, and the geometric means for the various clastic rock types are connected with a line. Perhaps the most obvious feature of the element patterns in figure 8 is a striking similarity of the minor element patterns to that of the illite-sericite-chlorite component of these same rocks (fig. 7). These patterns are like that discussed by Krauskopf (1955) but are based on a three-unit system (mudstone, siltstone, sandstone) instead of a two-unit system (shale, sandstone).

Elements that are enriched in the illite-sericite-chlorite-bearing clastic rocks from both areas apparently include $\mathrm{B}, \mathrm{Co}, \mathrm{Cr}, \mathrm{Ni}, \mathrm{Sc}, \mathrm{V}, \mathrm{Fe}, \mathrm{Mg}$, and $\mathrm{Ti}$, and possibly $\mathrm{La}$ and $\mathrm{Y}$. As these rocks contain no organic compounds, which might form organo-metallic complexes, the elements are probably contained in or adsorbed on the lattice of the micaceous minerals. Copper is more abundant in silty layers of laminated rocks or in siltites and quartzites of the Mission Mountains, and zircon appears concentrated in the coarser rocks in the Striped Peak Formation of the Pend Oreille area. This suggests that $\mathrm{Cu}$ and $\mathrm{Zr}$ are primarily in grains rather than held in clay minerals in those rocks. $\mathrm{Mn}, \mathrm{Pb}$, and $\mathrm{Sr}$ are more abundant in the rocks of the Mission Mountains where the $\mathrm{Ca}$ content is higher; such associations are common (Mason, 1956, p. 155; Tourtelot, 1962, p. 60) and suggest that some aragonite was originally deposited as part of the carbonate minerals. The higher $\mathrm{Mg}$ of the Mission Mountains reflects, in part, the more dolomitic character of these rocks. Similarr rock types of the Mission Mountains contain about twice as much $\mathrm{Co}, \mathrm{Cu}$, and $\mathrm{Ni}$ as their counterparts in the Pend Oreille area. The siltites and quartzites of the Pend Oreille area have a B content distinctly higher than similar rocks of the Mission Mountains.

Tables 6 and 7 show that the metamorphically low-grade rock types within each formation of each area contain similar concentrations of minor elements. The unusually high B content of the Striped Peak Formation, particularly of members 2 and 3 (table 7), is clearly limited to that formation and has displaced upward the graph for B in Pend Oreille rocks in 
figure 8. Low-grade rocks of other formations in the Pend Oreille area have the approximate B content of similar rocks in the Mission Mountains.

Comparison of the minor element content of Belt argillitic rocks with that of the Pierre Shale is also shown in figure 8; geometric means for the Pierre were calculated from data given by Tourtelot (1962, fig. 13). Most minor element contents of the Belt mudstones are similar to those of the Pierre Shale. The Pierre is relatively high in $\mathrm{Cr}, \mathrm{Cu}, \mathrm{Sr}$, and $\mathrm{V}$; $\mathrm{Sr}$ is correlated with $\mathrm{Ca}$ in the Pierre (Tourtelot, 1962, p. 60); and $\mathrm{Cr}, \mathrm{Cu}$, and $\mathrm{V}$ are commonly enriched in black shales (Krauskopf, 1955, table 2; Vine, 1966, p. E29). Even though much of the Belt argillitic rock is black, the organic carbon of the usual black shale is missing, as is normal enrichment in black shale suites of trace elements.

Distribution of the elements among constituent minerals was further examined by heavy mineral studies of 45 samples of low-grade rocks from the Pend Oreille area. Heavy mineral fractions were obtained by a simple bromoform separation from ground samples. Splits of the original sample and the heavy and light fractions were analyzed spectrographically. Heavy mineral fractions ranged from 0.1 to 3 percent of the rocks; most contained about 1 percent. The heavy fractions commonly contain compound grains that are mostly silicate minerals enclosing tiny black specks (magnetite). One exceptional sample, a silty carbonate rock of fossil algal forms, contains 11 percent heavy minerals. The minor element content of each split of the original sample was compared with its content as calculated from its fractions and was found to be precise within the limits of the analytical method. Average relative concentrations of elements in the heavy mineral

TABLE 9.-Relative concentration of elements in the heaiy mineral fraction of some Belt rocks from the Pend Oreille area

[Number in column is the ratio: average amount in heavy fraction divided by average amount in light fraction; 1 indicates equal amounts in the light and heavy fractions. Number in parentheses is number of samples of rock type. ......., the amount of the element in the heavy fraction was below the sensitivity limit of the spectrograph]

\begin{tabular}{ccccc}
\hline Element & $\begin{array}{c}\text { Argillite } \\
(17)\end{array}$ & $\begin{array}{c}\text { Siltite } \\
(20)\end{array}$ & $\begin{array}{c}\text { Quartzite } \\
(5)\end{array}$ & $\begin{array}{c}\text { Carbonate rocks } \\
(3)\end{array}$ \\
\hline $\mathrm{B}$ & 1 & 1 & 10 & $1 / 2$ \\
$\mathrm{Ba}$ & 1 & 1 & $1 / 2$ & 1 \\
$\mathrm{Be}$ & 1 & 1 & 1 & 1 \\
$\mathrm{Co}$ & 5 & 5 & 5 & $\cdots$ \\
$\mathrm{Cr}$ & 1 & 4 & 10 & 1 \\
$\mathrm{Cu}$ & 10 & 20 & 30 & $\cdots$ \\
$\mathrm{Fe}$ & 2 & 3 & 5 & 3 \\
$\mathrm{La}$ & 3 & 2 & 5 & 1 \\
$\mathrm{Mg}$ & 1 & 1 & 1 & 1 \\
$\mathrm{Mn}$ & 5 & 5 & 20 & 1 \\
$\mathrm{Ni}$ & 5 & 5 & 20 & $\ldots$ \\
$\mathrm{Pb}$ & 3 & 5 & 5 & 5 \\
$\mathrm{Sc}$ & $1 / 2$ & $\ldots \ldots$ & $\ldots$ & $\ldots \ldots$ \\
$\mathrm{Sr}$ & 1 & 1 & 1 & 12 \\
$\mathrm{Ti}$ & 3 & 2 & 5 & 1 \\
$\mathrm{~V}$ & 1 & 1 & 7 & 1 \\
$\mathrm{Y}$ & 5 & 5 & 10 & 2 \\
$\mathrm{Zr}$ & 5 & 3 & & \\
\hline
\end{tabular}


fraction of various rock types are shown in table 9 . The ratios range from about $1 / 2$ to 2 times the average shown in the table, except for Mn which is about $1 / 5$ to 5 times the average. The relative concentration of $B$ in heavy mineral fractions of Wallace argillites and siltites and of Striped Peak siltites is about 3 (not shown in table 9).

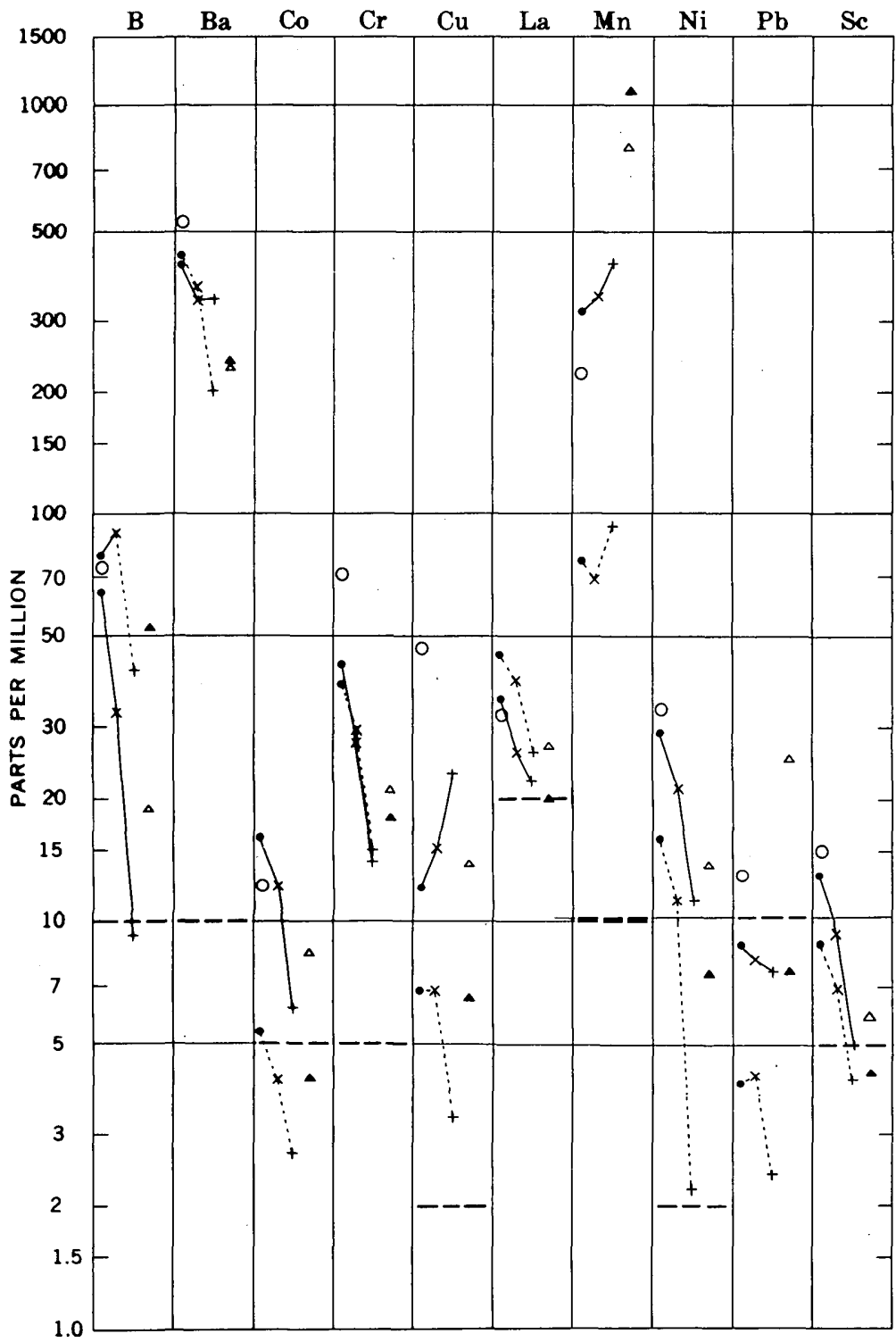

3

FIGURE 8.-Geometric means of semiquantitative spectrographic analyses of different Belt rock types. Number of samples from Mission Mountains: argillite, 62; siltite, 62; quartzite, 68; carbonate rocks, 62. Number of samples from Pend Oreille area: 


\section{EXPLANATION}

Belt argillite plus

laminated argil-

lite and siltite

$x$

Belt siltite

$+$

Belt quartzite

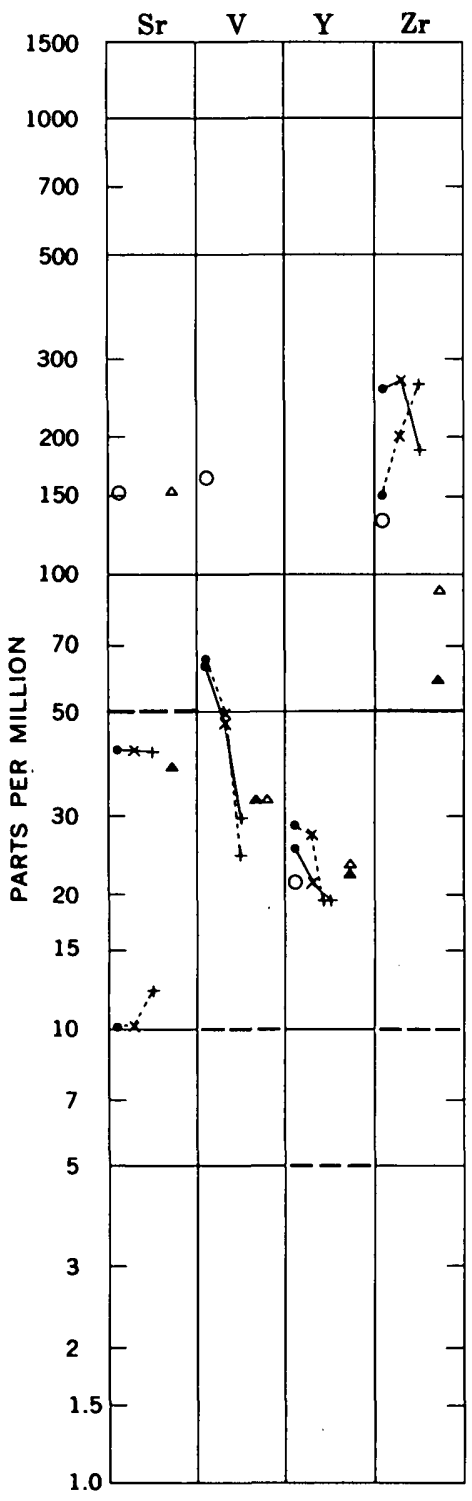

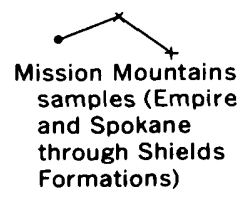

Formations)

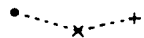

Pend Oreille area

samples (Revett

through Libby Formations)

\section{Spectrographic}

sensitivity limit

lower than smallest

value on graph

where not shown

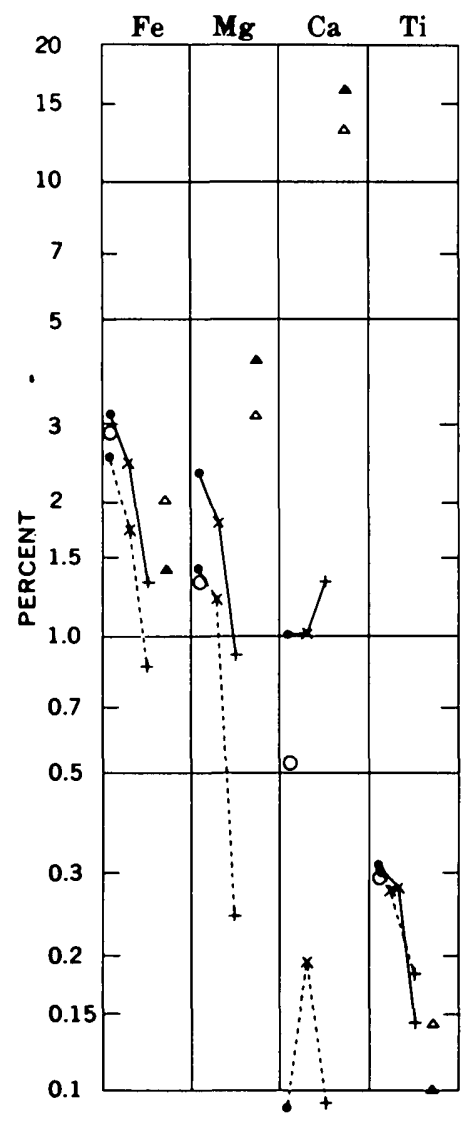

argillite, 48 ; siltite, 31 ; quartzite, 14 ; carbonate rocks, 13 . Also shown for comparison: Pierre Shale, 67 samples. 
The principal heavy minerals are magnetite, ilmenite, chalcopyrite, zircon, tourmaline, and iron hydroxides; some bornite, chalcocite, altered biotite, and manganese nodules occur in the coarser grained rocks. The copper minerals are largely in irregular or cup-shaped flakes, but some are in subhedral or broken grains; zircon, tourmaline, magnetite, and ilmenite are generally in broken or abraded crystals; some magnetite, ilmenite, and most pyrite are in discrete euhedral crystals. No significant differences in kinds of minerals in heavy mineral suites were observed for rocks of this study.

The concentration ratios (table 9) show clearly that many elements are about as abundant in the light fraction as in the heavy fraction. Because the light fraction forms about 99 percent of the rock, the bulk of most elements is associated with the principal rock-forming minerals. This then supports the interpretation that those elements shown in figure 8 that have a strong correlation in pattern with the pattern for illite-sericite-chlorite in figure 7 are adsorbed on or held within the lattice of those minerals.

Heavy mineral fractions of quartzites show relatively large concentrations of $\mathrm{B}, \mathrm{Cr}, \mathrm{Cu}, \mathrm{Mn}, \mathrm{Ni}$, and $\mathrm{Zr}$. $\mathrm{B}$ is in tourmaline; $\mathrm{Zr}$ is in zircon; $\mathrm{Cu}$ is in chalacopyrite, bornite, or chalcocite; $\mathrm{Mn}$ is in small blobs of probable manganese oxides; and $\mathrm{Cr}$ and $\mathrm{Ni}$ are probably associated with iron or manganese oxides (Krauskopf, 1955, p. 425).

Only copper shows a relatively large concentration in the heavy mineral fraction of all clastic rock types. The somewhat more abundant copper in rocks of the Mission Mountains (fig. 8) is in minerals that form enough of the rock to allow thin section study. Here the copper occurs as chalcopyrite grains and chalcocite flakes and blobs in unfractured rocks that suggest a detrital sedimentary origin; interstitial bornite blobs, however, suggest that diagenetic or other subsequent modification of any original copper is probable. The concentration and occurrence of copper in the heavy mineral fraction of Pend Oreille rocks support this conclusion.

\section{DISCUSSION AND INTERPRETATION IMPLICATIONS ABOUT SEDIMENTATION}

The average chemical composition of Belt rocks in the Pend Oreille area is between that of an average granite and that of the Canadian Shield (table 10). The weighted average for Belt rocks was computed from 23 chemical analyses of rocks from all formations in the Pend Oreille area where the stratigraphic section consists of about 80 percent pelitic rock, 15 percent quartzite, and 5 percent impure carbonate rock. Other parts of Belt terrane have a higher percentage of carbonate rock and perhaps slightly more quartzite. Thus the Belt rocks as a whole may be nearer the average composition of the Canadian Shield than is indicated in table 10. The Canadian Shield is, of course, one likely source terrane for Belt sediments.

Two lines of evidence point to a remarkable uniformity of depositional process lasting several hundred million years for Belt rocks. The direct 
TABLE 10.-Average granite and average composition of the Canadian Shield compared with average Belt rock from the Pend Oreille area

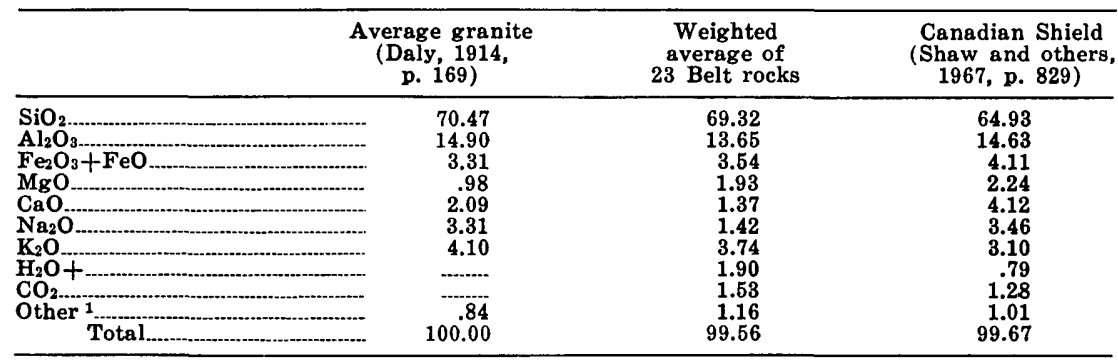

${ }_{1}^{1}$ Includes $\mathrm{H}_{2} \mathrm{O}-$, $\mathrm{TiO}_{2}, \mathrm{P}_{2} \mathrm{O}_{5}, \mathrm{MnO}, \mathrm{Cl}, \mathrm{F}-, \mathrm{BaO}, \mathrm{SrO}, \mathrm{C}$, and acid soluble $\mathrm{S}$ as $\mathrm{SO}_{8}$.

relation between mineralogy and rock grain size (fig. 6) implies a consistent weathering and transportation system. First, the almost total absence of life during deposition of Belt rocks certainly resulted in a simpler sedimentational history than in later geologic time. No plants covered the surface of the earth to impede erosion, and no humic acids were formed to accelerate leaching or to increase the variety of local weathering products. Little life existed in the oceans to produce clastic shell detritus or organic compounds which might combine with or alter mineral or chemical products brought in from the land. Second, the nearly uniform ratio among quartz, potassium feldspar, and plagioclase in widely separated sediments deposited over hundreds of millions of years requires either a nearly uniform source terrane or a high degree of integration of clastic components during transportation.

The chemical composition of the Belt seas and atmosphere was probably not much different from the composition of today's seas and atmosphere (Rubey, 1951, p. 1111). If the atmosphere of the time had been excessively high in $\mathrm{CO}_{2}$, "the rain water would have been so highly carbonated that it would leach much $\mathrm{Fe}$ and $\mathrm{Si}$ from exposed rocks, and the streams would transport significant amounts of those elements in solution ***" (Rubey, 1951, p. 1124). Because no iron-formations and only insignificant amounts of chert occur in the Belt rocks, it seems reasonable to assume that no great excess of $\mathrm{Fe}$ or $\mathrm{Si}$ was transported in solution to or accumulated in Belt areas.

The mineralogy and geochemistry of the Belt rocks suggest either that rocks of the source area were uniform in composition (near that of granite) or that the weathering and transportation processes homogenized the clastic assemblage prior to deposition. Homogenization during transportation is indicated by the remarkable uniformity of Belt rock mineralogy in a given rock type and of the ratio among quartz, potassium feldspar, and plagioclase in siltites. In the few places where the base of the Belt sequence is exposed, the rocks lie unconformably on older, crystalline rocks. Conceivably, part of the Belt strata may have been derived by reworking this base- 
ment or older adjacent strata of the thick Belt stratigraphic sequence. However, there are no known older sedimentary rocks that could have provided the tremendous volume of sediment of which the Belt is composed. Moreover, the increase in amount and grain size of feldspar in the upper parts of the supergroup, particularly in the feldspathic red quartzites of the Striped Peak and equivalent formations, suggests a marked change in provenance. The exposed crystalline basement of Montana and the Canadian Shield are not uniformly granitic and do contain mafic rocks. We are led to the conclusion that the weathering and transportation processes of Belt time resulted in homogenization of clastic components. This homogenization requires a chemical weathering of most mafic minerals, a low terrane and low-gradient streams carrying only fine-grained material, and a slow isostatic adjustment between source areas and basins to provide a reasonably stable cycle of erosion and deposition without major tectonic surges to cause folding or faulting within the basins. It is of some interest that the slow isostatic adjustment of a granitic crust over a long period of time resulting in widespread epicontinental seas is a corollary of Rubey's (1951,p. 1140-1142) thoughtful analysis of the geologic history of sea water.

The differences in minor element composition between similar rock types in various formations and in the two areas may be caused by a variation in depositional environment or by a variation in the source areas that supplied the Belt detritus. Carbonate is largely a chemical precipitate in these rocks; therefore, $\mathrm{Ca}$ and elements such as $\mathrm{Mg}, \mathrm{Mn}, \mathrm{Pb}$, and $\mathrm{Sr}$ that form carbonate compounds or substitute in mineral lattices of those compounds increase, as expected, in the environment of carbonate deposition.

Other minor elements showing large differences between the two areas are $\mathrm{B}, \mathrm{Co}$, and $\mathrm{Ni}$, and they are also related to the illite-sericite-chlorite fraction of the rocks. The high $\mathrm{B}$ content of the Striped Peak Formation, particularly members 2 and 3 , is in part a reflection of more abundant detrital tourmaline in these rocks than in others. The bulk of the B, however, in in the fine-grained light fraction and probably represents original B-rich clay minerals (Hirst, 1962, p. 1172-1176). Here, then, is an indication of a different source area for part of the Belt strata. The siltites of the formation below the Striped Peak are also somewhat enriched in tourmaline, which may indicate that the source area for these rocks was similar to that of the Striped Peak. If so, the homogenization of clastic components was more thorough in the lower (Wallace) strata.

$\mathrm{Co}$ and $\mathrm{Ni}$ are more abundant in the Mission Mountains rocks than in the Pend Oreille rocks. If these elements reflect a difference in crystalline source terrane, then we might also expect correspondingly large amounts of $\mathrm{V}, \mathrm{Cr}$, and $\mathrm{Cu}$ from rocks relatively high in Co and Ni (Parker, 1967, table 19). $\mathrm{Cu}$ is present in larger amounts in Mission Mountains rocks. It also is primarily in the form of sulfides in the rocks and thus differs from $\mathrm{V}$ and $\mathrm{Cr}$, 
which are largely with clay minerals. If we can accept the thesis of the existence of $\mathrm{Co}, \mathrm{Ni}, \mathrm{V}$, and $\mathrm{Cr}$ in a simple chemical environment, then the difference in their behavior may be based on ionic potential because $\mathrm{Cr}^{+3}>\mathrm{V}^{+3}$ $>>\mathrm{Ni}^{+2}>\mathrm{Co}^{+2}$, as measured for behavior of their hydroxides (Goldschmidt, 1937; Krauskopf, 1955, table 9). Thus $\mathrm{Cr}$ and V are in about equal amounts in similar rock types of the two areas because of their superior ability to substitute in lattices of the clay minerals which are in equal amounts in the comparable rock types. Excess $\mathrm{Ni}$ and $\mathrm{Co}$ are left to be absorbed or precipitated primarily in sulfides. Many simplifying assumptions on the chemistry of the environments are required to allow such statements to be made. But the hypothesis is reasonable in terms of the geologic data and permits one further suggestion. The source area for the Mission Mountains strata contained more basic rocks than that for equivalent strata of the Pend Oreille area. This has bearing on the origin of stratabound copper deposits or occurrences.

Copper in all these rocks is primarily in discrete sulfides that occur in greatest abundance in siltites and quartzites of the Mission Mountains. The original copper minerals occur primarily as heavy mineral grains and interstitial flakes, and some diagenetic recrystallization is probable (Harrison and others, 1969, p. D17). In these rocks, sedimentary copper was apparently deposited as heavy mineral grains in the coarser grained rocks or with stromatolites (fossil algal forms) or was subsequently concentrated in more permeable strata. The living algae formed sticky mats that were effective traps for heavy minerals. This is shown by the 11-percent heavy mineral fraction of a Pend Oreille algal sample and by the high clastic copper mineral content of a persistent zone of stromatolitic rocks in the Mission Mountains (Harrison and others, 1969, p. D18). Several Pend Oreille stromatolites examined in thin section tend to have high heavy mineral content, which is mostly clastic grains of magnetite-ilmenite. Apparently, clastic grains of copper minerals were not available in the Pend Oreille area environment at the time of deposition of the stromatolitic rocks. Bornite flakes do occur as interstitial fillings in some quartzites and siltites, but in markedly less amounts than in the Mission Mountains strata.

We did not see a syngenetic copper ore deposit in either study area, although copper may occur in concentrations up to 3 percent in some 1-inch-thick beds. Although part or all of the chalcopyrite and perhaps some chalcocite may be syngenetic, the bornite is not; it is either diagenetic or epigenetic. Because the Helena and younger Belt rocks probably contain more syngenetic copper in the eastern part of the Belt terrane, prospecting for copper in these rocks may be more fruitful in the eastern part of the old Belt basin. We have no data on distribution or occurrences of copper among various areas or formations in the lower (pre-Helena) part of the Belt rocks. 


\section{METAMORPHIC CHANGES}

Samples from the Pend Oreille area fall into three subfacies of metamorphism within the greenschist facies. They represent the chlorite-sericite zone and the biotite zone of regional metamorphism $(\mathrm{C}$ and $\mathrm{Bb}$ in tables 1 and 7), and the biotite zone of contact metamorphism (Bgn in tables 1 and 7$)$.

Chemical changes between the chlorite-sericite and biotite zones of regional metamorphism are shown in table 7 . Here we can look only for similar patterns or trends within different formations because of the limited numbers of samples in some groups, large values for geometric deviation, and limits of the analytical method. We conclude that the trends support Shaw's (1956, p. 934) general conclusion that regional metamorphism of shales results in little change in minor element content. One trend is suggested in our data-a loss of boron with increasing metamorphic grade. Boron was not included in Shaw's studies, and so a comparison is not possible.

Effects of contact metamorphism can be examined only in the Prichard Formation. Within this formation, the argillitic rocks give the best measure of metamorphic change because (1) a larger number of samples were collected of this rock type, (2) metamorphic equivalents are more easily recognized, and (3) among the lower-grade rocks, the Prichard argillitic rocks are more nearly uniform in mineralogy from place to place than are the siltites and quartzites. The higher grade argillitic rocks show an increase in $\mathrm{Ca}$ and $\mathrm{Sr}$ and a decrease in $\mathrm{Ni}, \mathrm{B}$, and possibly Co. Co and $\mathrm{Ni}$ are commonly associated, as are $\mathrm{Ca}$ and $\mathrm{Sr}$. The B loss with increasing metamorphism supports the trend identified in the regional metamorphism. Other elements show no detectable change. Further studies of these metamorphic effects are underway.

\section{COMPARISON OF BELT ROCKS WITH YOUNGER ROCKS}

Table 11 compares the element content of the Mission Mountains and Pend Oreille pelitic rocks with that of similar rocks of different ages and geologic environments. The Ordovician rocks are highly siliceous eugeosynclinal sedimentary rocks; their geometric means have been calculated from analyses given by Ketner (1969, tables 3, 4). The Devonian rocks are largely metamorphosed, and the geometric means were calculated from analyses given by Shaw $(1954$, tables $7,10,13)$ by assigning his quantitative spectrographic data to the appropriate semiquantitative spectrographic classes used to calculate geometric means for the other groups of rock shown in table 11. The Cretaceous rocks are the marine Pierre Shale, and the geometric means were calculated from analyses given by Tourtelot (1962, 
TABLE 11.-Average (geometric means) minor element content of pelitic rocks of different ages and geologic environments

[Results in parts per million. ........, insufficient or no data]

\begin{tabular}{|c|c|c|c|c|c|}
\hline \multirow[b]{2}{*}{ Element } & \multicolumn{2}{|c|}{ Precambrian } & \multirow{2}{*}{ 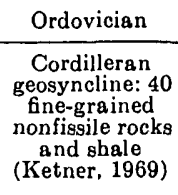 } & \multirow{2}{*}{ 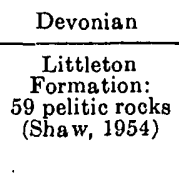 } & \multirow{2}{*}{$\begin{array}{c}\text { Cretaceous } \\
\text { Pierre Shale } \\
67 \text { shales } \\
\text { (Tourtelot, } \\
1962)\end{array}$} \\
\hline & $\begin{array}{c}\text { Mission } \\
\text { Mountains } \\
\text { Primitive Area: } \\
124 \text { pelitic } \\
\text { rocks } \\
\text { (this report) }\end{array}$ & $\begin{array}{l}\text { Pend Oreille } \\
\text { area: } 79 \\
\text { pelitic rocks } \\
\text { (this report) }\end{array}$ & & & \\
\hline $\begin{array}{l}\mathrm{B} \\
\mathrm{Ba} \\
\mathrm{Co} \\
\mathrm{Cr} \\
\mathrm{Cu} \\
\mathrm{La} \\
\mathrm{Mn} \\
\mathrm{Ni} \\
\mathrm{Pb} \\
\mathrm{Sc} \\
\mathrm{Sr} \\
\mathrm{V} \\
\mathbf{Y} \\
\mathrm{Zr}\end{array}$ & $\begin{array}{c}46 \\
370 \\
14 \\
35 \\
14 \\
30 \\
330 \\
25 \\
8.4 \\
11 \\
42 \\
54 \\
23 \\
260\end{array}$ & $\begin{array}{c}83 \\
400 \\
4.8 \\
34 \\
6.8 \\
43 \\
74 \\
14 \\
4.0 \\
7.9 \\
25 \\
58 \\
27 \\
170\end{array}$ & $\begin{array}{r}72 \\
770 \\
52 \\
47 \\
220 \\
15 \\
\overline{-} \\
61 \\
\frac{60}{74}\end{array}$ & $\begin{array}{r}\overline{-} \\
18 \\
110 \\
9 \\
\bar{Z} \\
\overline{57} \\
18 \\
12 \\
630 \\
110 \\
41 \\
190\end{array}$ & $\begin{array}{r}73 \\
520 \\
12 \\
70 \\
46 \\
32 \\
220 \\
33 \\
13 \\
15 \\
150 \\
160 \\
21 \\
130\end{array}$ \\
\hline
\end{tabular}

table 14). Data on the Mission Mountains and Pend Oreille rocks are given in tables 6 and 7 of this report.

Both the Ordovician and Cretaceous rocks contain abundant black shales, and, to choose Krauskopf's (1955, p. 418), careful phrasing, "some elements show a tendency to concentrate***" in this particular rock type. The elements listed in table 11 that may show this tendency are $\mathrm{Cr}, \mathrm{Cu}, \mathrm{Ni}, \mathrm{Pb}$, and V (Krauskopf, 1955, table 2). Most of them are more abundant in the Ordovician and Cretaceous rocks than in the Belt rocks. One can cautiously infer that the metamorphosed pelitic rocks of the Devonian Littleton Formation of the Eastern United States originally included black shales, because they are enriched in all but $\mathrm{Cu}$ relative to the non-organic Precambrian pelitic rocks. Tourtelot (1962, p. 50-51) noted the close similarity in minor element content between the Pierre Shale and the Littleton Formation, but he chose not to infer that the close relation might be due to similarities among black shales. Perhaps the minor element content of Belt rocks more nearly represents that to be expected in pelitic rocks containing little or no organic material.

\section{CONCLUSIONS}

Relatively inexpensive techniques of quantitative X-ray mineralogic determinations and semiquantitative spectrographic chemical analyses have provided data adequate for meaningful geologic interpretation of Belt rock sedimentation processes. The principal difference in mineralogy of three types of Belt clastic rocks involves presence or absence of carbonate cement, and this is reflected in the chemistry of those rocks containing 
carbonate minerals by an increase in $\mathrm{Ca}, \mathrm{Mn}, \mathrm{Sr}$, and $\mathrm{Pb}$. The abundance of many elements is directly related to the abundance of the illite-sericitechlorite fraction in the rock. Relative abundances of many elements in this fraction reflect ionic potential of the elements. Other differences in minor element chemistry are inferred to reflect source terrane. The occurrence and distribution of copper are the most significant in terms of economic geology. The more abundant copper appears related to source area of the sediments. Within a broad geographic area, certain strata may either contain stratiform deposits or represent source beds for secondary deposits.

The data obtained by using these techniques do not identify any metamorphic changes from the chlorite-sericite zone to the biotite zone of regional metamorphism except for a probable loss of boron. Contact metamorphism also in the biotite zone but resulting in formation of schists and gneisses was accompanied by an increase in $\mathrm{Ca}$ and $\mathrm{Sr}$ and a decrease in $\mathrm{Ni}, \mathrm{B}$, and possibly Co. The minor element content of pelitic Belt rocks is probably representative in general of nonorganic pelitic rocks as contrasted with black shales or pelitic rocks containing black shales as studied by others (Shaw, 1954; Tourtelot, 1962; Vine, 1966; Ketner, 1969).

\section{REFERENCES CITED}

Anderson, A. L., 1930, Geology and ore deposits of the Clark Fork district, Idaho: Idaho Bur. Mines and Geology Bull. 12, 132 p.

Bayley, R. W., and Muehlberger, W. R., compilers, 1968, Basement rock map of the United States (exclusive of Alaska and Hawaii) : U.S. Geol. Survey map.

Daly, R. A., 1914, Igneous rocks and their origin: New York, McGraw-Hill Book Co., $563 \mathrm{p}$.

Fleischer, Michael, 1965, Summary of new data on rock samples G-1 and W-1, 1962-65: Geochim. et Cosmochim. Acta, v. 29, no. 12, p. 1263-1283.

Goldschmidt, V. M., 1937, Principles of distribution of chemical elements in minerals and rocks: Chem. Soc. London Jour., p. 655-673.

Grimes, D. J., and Marranzino, A. P., 1968, Direct-current arc and alternatingcurrent spark emission spectrographic field methods for semiquantitative analysis of geologic materials: U.S. Geol. Survey Circ. 591, 6 p:

Harrison, J. E., and Campbell, A. B., 1963, Correlations and problems in Belt Series stratigraphy, northern Idaho and western Montana: Geol. Soc. America Bull., v. 74, no. 12 , p. 1413-1427.

Harrison, J. E., and Jobin, D. A., 1963, Geology of the Clark Fork quadrangle, IdahoMontana: U.S. Geol. Survey Bull. 1141-K, 38 p.

Harrison, J. E., Reynolds, M. W., Kleinkopf, M. D., and Pattee, E. C., 1969, Mineral resources of the Mission Mountains Primitive Area, Missoula and Lake Counties, Montana: U.S. Geol. Survey Bull. 1261-D, 48 p.

Hirst, D. M., 1962, The geochemistry of modern sediments from the Gulf of Paria[Pt.] 2, The location and distribution of trace elements: Geochim. et Cosmochim. Acta, v. 26, p. 1147-1187. 
Ketner, K. B., 1969, Ordovician bedded chert, argillite, and shale of the Cordilleran eugeosyncline in Nevada and Idaho, in Geological Survey research 1969: U.S. Geol. Survey Prof. Paper 650-B, p. B23-B34.

Krauskopf, K. B., 1955, Sedimentary deposits of rare metals, pt. 1 of Bateman, A. M., ed., Economic geology - 50th anniversary volume, 1905-55: Urbana, Ill., Econ. Geology Pub. Co., p. 411-463.

McGill, G. E., and Sommers, D. A., 1967, Stratigraphy and correlation of the Precambrian Belt Supergroup of the southern Lewis and Clark Range, Montana: Geol. Soc. America Bull., v. 78, no. 3, p. 343-351.

Mason, B. H., 1956, Principles of geochemistry [1st ed.]: New York, John Wiley \& Sons, Inc., $276 \mathrm{p}$.

Maxwell, D. T., and Hower, John, 1967, High-grade diagenesis and low-grade metamorphism of illite in the Precambrian Belt Series: Am. Mineralogist, v. 52, nos. 5-6, p. 843-857.

Miesch, A. T., 1967, Methods of computation for estimating geochemical abundance: U.S. Geol. Survey Prof. Paper 574-B, 15 p.

Mudge, M. R., 1970, Origin of the disturbed belt in northwestern Montana: Geol. Soc. America Bull., v. 81, no. 2, p. 377-392.

Obradovich, J. D., and Peterman, Z. E., 1968, Geochronology of the Belt Series, Montana: Canadian Jour. Earth Sci., v. 5, no. 3, pt. 2, p. 737-747.

Parker, R. L., 1967, Composition of the earth's crust, in Fleischer, Michael, ed., Data of geochemistry [6th ed.]: U.S. Geol. Survey Prof. Paper 440-D, 19 p.

Ross, C. P., 1963, The Belt series in Montana, with a geologic map compiled by B. A. L. Skipp, and a section on Paleontologic criteria, by Richard Rezak: U.S. Genl. Survey Prof. Paper 346, 122 p. [1964].

Rubey, W. W., 1951, Geologic history of sea water: Geol. Soc. America Bull., v. 62, . no. 9, 1111-1147.

1956, Major elements and general geochemistry, pt. 3 of Geochemistry of pelitic rocks [N.H.]: Geol. Soc. America Bull., v. 65, no. 12, p. 1151-1166.

1956 Major elements and general geochemistry, pt. 3 of Geochemistry of pelitic rocks: Geol. Soc. America Bull., v. 67, no. 7, p. 919-934.

Shaw, D. M., Reilly, G. A., Muysson, J. R., Pattenden, G. E., and Campbell, F. E., 1967, An estimate of the chemical composition of the Canadian Precambrian Shield: Ganadian Jour. Earth Sci., v. 4, no. 5, p. 829-853.

Tatlock, D. B., 1966, Rapid modal analysis of some felsic rocks from calibrated X-ray diffraction patterns: U.S. Geol. Survey Bull. 1209, 41 p.

Tourtelot, H. A., 1962, Preliminary investigation of the geologic setting and chemical composition of the Pierre shale, Great Plains region: U.S. Geol. Survey Prof. Paper 390, 74 p.

Vine, J. D., 1966, Element distribution in some shelf and eugeosynclinal black shales: U.S. Geol. Survey Bull. 1214-E, p. E1-E31. 